\title{
Mathematical modeling of morphogenesis in living materials
}

\author{
Valentina Balbi and Pasquale Ciarletta
}

\begin{abstract}
From a mathematical viewpoint, the study of morphogenesis focuses on the description of all geometric and structural changes which locally orchestrate the underlying biological processes directing the formation of a macroscopic shape in living matter. In this chapter, we introduce a continuous chemo-mechanical approach of morphogenesis, deriving the balance principles and evolution laws for both volumetric and interfacial processes. The proposed theory is applied to the study of pattern formation for either a fluid-like and a solid-like biological system model, using both theoretical methods and simulation tools.
\end{abstract}

\section{Introduction}

In developmental biology, morphogenesis can be defined as the ensemble of the underlying biological processes directing the formation of a macroscopic shape in living matter. Morphogenetic events span over a wide range of molecular and cellular mechanisms, often inter-playing for controlling events at individual or collective level. Thus, the shape of an organism emerges over time as a morphological transition coordinated by these complex multi-scale interactions, revealing the intrinsic dynamic nature of its driving forces. Indeed, from a mathematical viewpoint, the study of morphogenesis focuses on the description of all geometric and structural changes which locally orchestrate the occurrence of a global shape in living mate-

Valentina Balbi

Sorbonne Universités, Université Paris 6, Institut Jean le Rond d'Alembert, UMR 7190, 4 place Jussieu case 162, 75005 Paris, France. e-mail: valentina.balbi@etu.upmc.fr

Pasquale Ciarletta

CNRS and Sorbonne Universités, Université Paris 6, Institut Jean le Rond d'Alembert, UMR 7190, 4 place Jussieu case 162, 75005 Paris, France

Mox, Dipartimento di Matematica, Politecnico di Milano, piazza Leonardo da Vinci 33, 20133

Milano, Italy. e-mail: pasquale.ciarletta@upmc.fr, pasquale.ciarletta@polimi.it 
rials. Accordingly, a mathematical model must consider the two most fundamental processes during tissue development, namely growth and remodeling, intended as the biological mechanisms determining any variation of the mass and of the microstructural organization, respectively [111]. Nonetheless, morphogenesis is by far a more complex phenomenon, since these basic mechanisms often occur simultaneously, involving a cascade of inter-related developmental events from the molecular to the tissue scale, provoked by either genetic and epigenetic causes [31].

It is therefore mandatory for a mathematical theory of morphogenesis to provide a dynamic modelling of the morphology as a function of the interplay between geometry and physical forces, as well as chemical and biomolecular cues. Moreover, the suitability of a mathematical model should be evaluated not only for the accuracy of reproducing the observed morphology in the dynamical fashion, but also for the ability to identify the most relevant underlying causes, i.e causas alias ex aliis aptas et necessitate nexas ${ }^{1}$.

Before investigating in details the main building blocks for a suitable mathematical modelling, we briefly make an overview about the development of the morphogenetic theories over the centuries, highlighting the path towards the contemporary vision of morphogenesis and its most intriguing open challenges.

\section{An historical overview of morphogenetic theories}

The scope of this section is to provide an historical survey of the main events concerning evolutionary theories and biological discoveries, from the ancient times to the modern and contemporary approaches.

\subsection{Epigenesis versus pre-formationism: from ancient times to the advent of microscopy}

Although it is known that Babylonians and Egyptians made an earlier use of geometrical calculations for structural purposes, the first application of geometry for explaining the origin of a shape can be found in Euclid's Elements (Book VI, definition 3, ca.300 BC), where "a straight line is said to have been cut in extreme and mean ratio when, as the whole line is to the greater segment, so is the greater to the less". This definition highlighted a simple proportion already used almost two centuries in advance by the sculptor Phidia, who seemingly used it to construct the Parthenon's statues. The possibility of using such a simple geometrical rule to decrypt the harmony and symmetry behind several shapes commonly observed in Nature still creates much fascination in our times, explaining the Medieval belief

${ }^{1}$ In mathematical terms, this latin expression can be roughly translated as the set of the strictly necessary causes (from Cicero, Rhetorica, Tusculanae Disputationes, Liber Quintus, 70). 
of a divine number. ${ }^{2}$. Indeed, the theological doctrine of creationist prevailed for almost two millennia, and shape was considered as a pre-ordered organization of matter resulting from an act of divine creation. Opposed to such a pre-formationist view, Aristotle had already proposed in his books Hystoria Animalium and De Generatione Animalium (ca.350 $\mathrm{BC}$ ) the so-called epigenetic hypothesis, according to which the growing tissues undergo after several steps of differentiation, forming structures which were not present at the initial steps of life [6]. Such works were mainly based on teleological concepts, but they reported an impressive number of biological observations on embryos, although methodologically very far from a rigorous modern scientific approach.

Notwithstanding, the predominance of preformationism persisted until the 17 th Century, where the advent of microscopy techniques opened a scientific debate between epigenetists and preformationists about the nature of embryonic structures. From one side, William Harvey retraced the Aristotelian ideas proposing the principle ex ovo omnia, i.e. that everything develops epigenetically from an egg [60]; on the opposite side, Marcello Malpighi and Nicolaas Hartsoeker claimed that a miniature structure of the adult organism, called homunculus, was present in the egg from the very beginning, and that the gestation period involved the growth and unfolding of that pre-existing structure $[1,59]$.

Few decades in advance, Galileo Galilei had hinted that the shape of organisms was somehow influenced by the load that they have to hold, proving that the changes in size of bones over mammals are actually governed by allometric scaling [47]. This seminal idea of mechanical causation would still take almost two centuries before influencing the scientific view of morphogenesis.

\subsection{The birth of modern embryology: evolutionary theories and mechanical causation}

The 19th century was dominated by Darwinism and the advent of genetics, but also by the theory that plants and organisms are made of cells, and that reproduction is governed by cell division, according to the principle omnis cellulae ex cellula proposed by Rudolf Virchow [116]. Furthermore, the technical progress in microscopy determined the birth of the modern experimental embryology. Inspired by Darwinism, Ernst Haeckel proposed the recapitulation theory, claiming that the developmental steps of embryonic growth would be a reflection of the adult evolution of their ancestors [55]. This biogenetic law formulated that ontogeny (i.e. the development of an individual organism) recapitulates phylogeny (i.e. the evolution of a species). Although immediately rejected by Wilhelm His, who showed with experiments that the gut tube morphogenesis could be modeled by a mechanical causation principle [93], this biogenetic theory prevailed in the scientific community until

2 This idea was enforced by Luca Pacioli, a student of Leonardo da Vinci, in his book De divina proportione, published in 1509 
the works of Wilhelm Roux towards the end of the 19th century. In particular, he shifted the focus of developmental biology from evolution (i.e. the final purpose) to the underlying mechanisms (i.e. the causes), performing several experiments on embryos in order to investigate the self-differentiation [100]. Although some of his deductions were incorrect [56], Roux inspired a new approach in experimental embryology, giving seminal results about regulation, i.e. the ability of the the embryo to develop normally even if a part of it is removed, as well as induction, i.e. the influence of a cell or a tissue on the development of another. Interestingly, a definitive confirmation of the latter phenomenon had to wait till 1924, when Spemann and Mangold transplanted part of the embryonic tissue from a amphibian into an embryo of a different amphibian species [107]. They observed that a partial second embryo developed from the transplanted tissue: the hosting tissue induced the development of the hosted tissue.

Roux also extended the investigation about the mechanical causation of development establishing the concept of functional adaptation as a principle for dependentdifferentiation: cells and tissues respond to change in external conditions in order to preserve their global organization and functions. Accordingly, any tissue would adapt under the action of external forces, regulating its growth order to preserve lightness and structural robustness.

This fundamental principle contributed to the development of modern physiology, which considers that all life phenomena result from the mutual balance between the living matter and the surrounding media. In the same period, indeed, Claude Bernard first hypothesized that such a dynamic equilibrium might regulate living organisms despite the structural complexity of the constituting matter [10]. Initially focusing on anatomical- biochemical and symphato-adrenal stimuli, novel experiments highlighted the stable equilibrium be- tween the interior matter of a living entity in the face of external perturbative agents. Accordingly, this physiological tendency towards a steady state was later called homeostasis, and later found to drive the optimal structural remodelling of both bone to mechanical loading, also known as Wolff's law [118], and arterial endothelia to shear stress, also known as Murray's law [90].

\subsection{The contemporary approches to morphogenesis}

The 20th century has been particularly rich in crucial discoveries and scientific contributions to the understanding of morphogenesis. In particular, mathematical modelling emerged as a In particular, the first half of the century was characterized by the introduction of mathematical modelling to investigate the relations between the physical forces exerted by the surroundings and the steady generation of shapes. The second half was instead dominated by the many astonishing discoveries in genetics and molecular biology, shifting the research focus to the dynamical aspects of morphogenesis. 


\subsubsection{The first mathematical approach on growth and form}

In 1917, D'Arcy Wentworth Thompson published his masterpiece and only scientific book, entitled On growth and form [112], a compendium of more than a thousand pages, written in wartime, about the use of mathematical theory to describe the shape of living organisms.

While other contemporary scientists focused on experimental analysis, D'Arcy Thompson's investigations were purely based on mathematical background. Being skeptical about a morphogenetic analysis based on evolution theories and natural selection, which were still dominant in the scientific community at that time, his original idea of morphogenetic development was instead focused on the role of physical forces in shaping organisms. The following quote from the Introduction section is fully explicative in this sense:

It is retained, somewhat crudely, in modern embyology

[...] that the embryonic phenomena must be referred directly

to their usefulness in building up the body of the future animal which is no more, and no less, to say, with Aristotle, that the organism is the $\tau \varepsilon \lambda$ o $\varsigma$, or final cause,

of its own processes of generation and development.

[...] Still, all the while, like warp and woof, mechanism and teleology

are interwoven together, and we must not cleave to the one nor despise the other;

for their union is rooted in the very nature of totality.

Accordingly, the book investigates mechanical causation in the generation of many shapes in living matter, dealing with living organisms as material bodies subjected to physical forces and obeying to simple physical and geometrical laws. An illustrative example of the originality of D'Arcy Thompson's approach can be found in the second chapter of his book, where he focused on the physical scaling effects on the shape of animals of different sizes, complementing Galileo's first observations on allometric laws. He proved that, whilst big animals must have strong and heavy structures which allow to sustain the bulk gravitational load, the shapes and structures small animals can be calculated from a principle of functional adaptation to surface forces, e.g. surface tension. Furthermore, he proposed a grid transformation method, aimed at showing that physical forces can shape a living organism during either growth or evolution, as depicted in Figure 1. D'Arcy Thompson's work had a seminal influence on modern biomechanics and his book is still read and published in reduced and revised versions. In between many other examples, it was a source of inspiration for the Huxley's work on allometric growth [67] as well as for Gould's mechanistic theory of shape from an evolutionary viewpoint [51].

\subsubsection{The chemical bases of morphogenesis}

The fast development of experimental techniques in biochemistry promoted an increasing attention to the chemical and molecular mechanisms underpinning mor- 
(a)
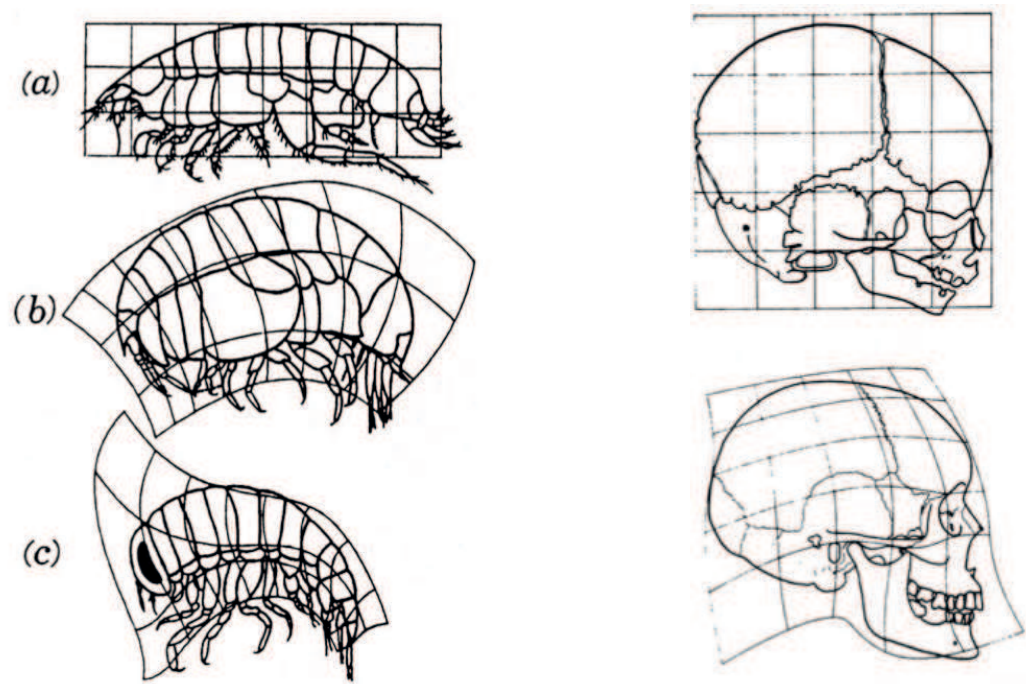

Fig. 1 Left: Transformation grid applied to the shape transformation of a small amphiopod (a) Harpinia Plumosa into the shapes of two other genera belonging to the same family (b) Stegocephalus Inflatus, and (c) Hyperia Galba), adapted from [29]. Right: Transformation grid applied to the growth of a skull in human foetus. In both examples the transformation is achieved by applying physical forces on the considered structure, during evolution and growth respectively.

phogenetic processes. A new branch of applied mathematics especially focused on the description of pattern formation, i.e. the emergence of organized living structures in space and time. A milestone in biomathematics is the paper by Alan Turing on the chemical bases of morphogenesis, published in 1952 [114]. Although it was his only contribution on morphogenesis, it is an extraordinary innovative work, especially since it proved how a mathematical theory could actually anticipate biological discoveries. Turing proposed a reaction-diffusion model of at least two chemical species which undergo chemical reaction within a living material: in order to highlight their role in generating a new pattern, he called them morphogens. They practically act biochemical substances enabling certain shape control abilities in the embryonic tissue, closely resembling the evocators earlier hypothesized by Waddington [117]. In the absence of diffusion, the biological system is in a stable state defined by homogeneous concentrations of the two reactants. Under certain range of values of the reaction and diffusion parameters, an instability occurs and a stable non-homogenous pattern arises. This was counter-intuitive result since it was thought that diffusion would rather introduce a chaotic behavior in the system, instead of generating an organized pattern.

In particular, Turing's model predicted the existence of six possible steady-states as shown in Figure 2. The uniform stationary (I) and oscillatory (II) states, the short wavelengths stationary (III) and oscillatory (IV) states and the finite wavelength stationary (V) and oscillatory (VI) states. Of particular interest is the Case VI, which 

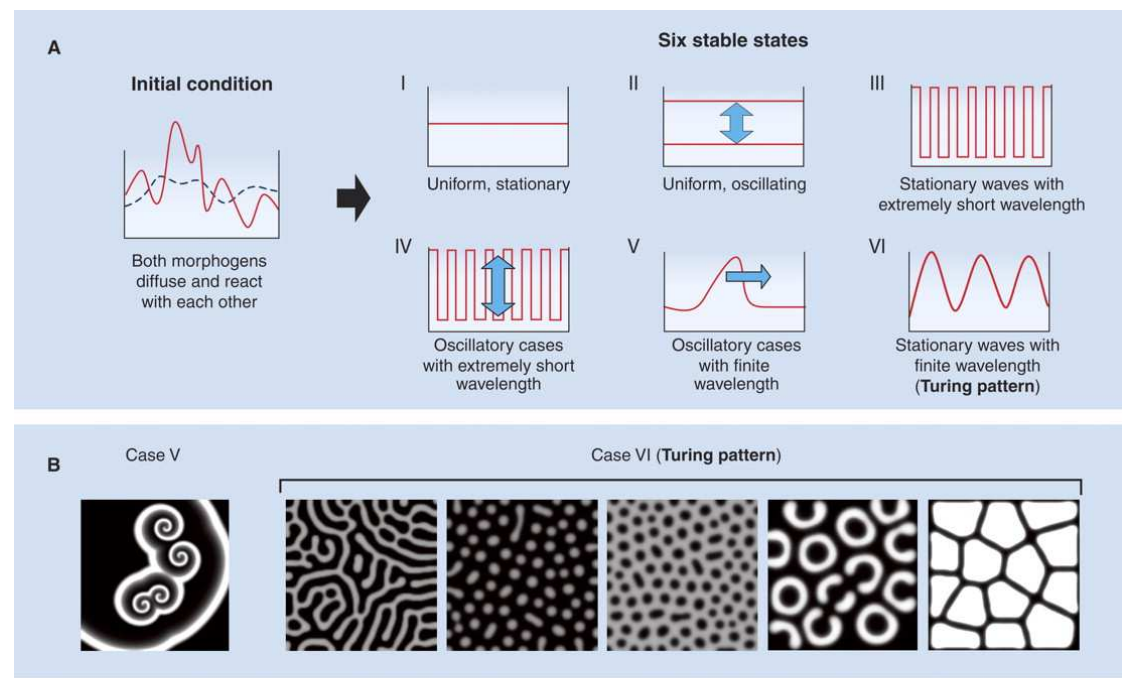

c
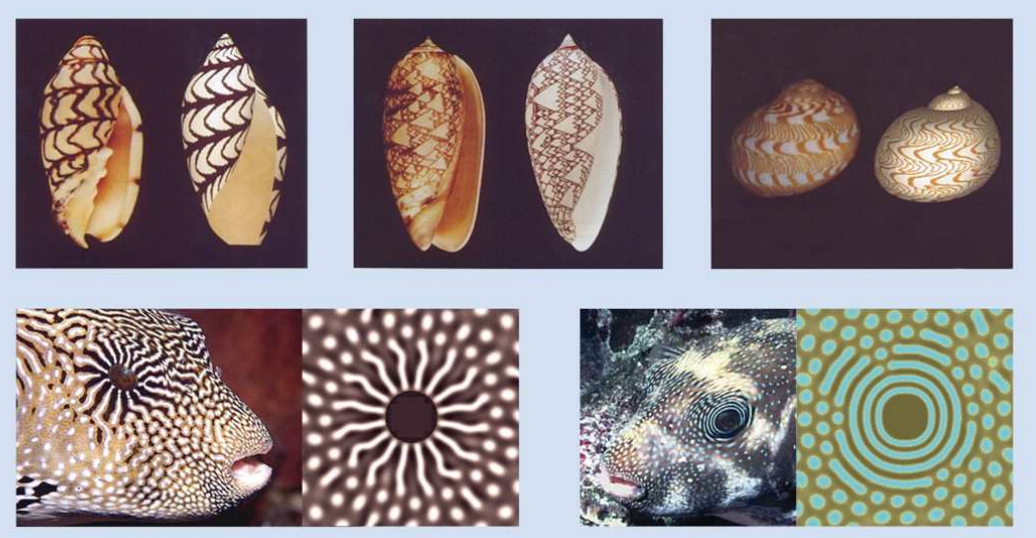

Fig. 2 Turing's reaction-diffusion model: (a) Examples of the six stable states solutions of Turing's model. (b) The so-called Turing's pattern is depicted as the Case VI where a stationary wave of finite wavelength develops. (C) Reproduction of biological patterns created by modified reactiondiffusion mechanisms. Adapted from [73].

occurs when the diffusion coefficients of the two morphogens differ substantially and initiate the so-called short-range activation, long-range inhibition [48] mechanism. The two morphogens are seen as an activator and an inhibitor, respectively, which can act on themselves as well as on the other. A small perturbation in the homogeneous concentration can induce an increase in the activator concentration and initiate the feedback which lead to the formation of one of the Turing's patterns in Figure 2 (b).

Turing's model has later been largely employed for modeling the emergence of sev- 
eral patterns in vertebrates such as the stripes in the zebra-fish pigmentation [72], the branching pattern in feathers [58], but also the fabulous seashell patterns [95] and the mechanism of plant phyllotaxis, i.e. the arrangement of leaves on a plant stem [106].

Nonetheless, Turing's work did not receive a great attention in the years right after its publication. Indeed, the problem of emergence of organized cellular patterns in the tissue was brought back to the attention of developmental biologists only in the 1970s, when Lewis Wolpert introduced the concept of positional information (PI). The main idea of Wolpert is that the position of a cell in the tissue specifies the information about the molecular changes the cell will undergo [119]. In this sense, morphogens act convey both positional information and growth orchestration properties. The key elements of Wolpert's theory can be summarized in the definition of:

- A mechanism which specifies the polarity in the tissue. Polarity is the direction in which PI is specified and is defined with respect to one or more reference points.

- A mechanism for specifying the different responses of the cell.

PI can be specified by a quantitative variation of some factor such as the concentration, or a qualitative variation of some cell parameters such as a combination of genes or enzymes. A set of cells which have their PI specified with respect to the same reference points constitutes a field. Interpretation of PI is the process by which PI specifies the cell state and conversion is the mechanism by which PI is translated in a particular cellular activity. Furthermore, PI is universal in organisms and size invariant, meaning that if a part of the tissue is removed, the tissue is still able to pattern and interpret the PI.

The concept of positional information is well clarified in the French flag model depicted in Figure 3. In this example, the mechanism which specifies polarity is the monotonic variation of the morphogen concentration $C . T_{1}$ and $T_{2}$ identify the mechanism for the differential response of the cells. The interpretation acts according to the following rule: cells with position in the region where $C<T_{1}$ express the blue pigment, a cell in the region where $T_{1}<C<T_{2}$ expresses the white pigment and cells in the region where $C>T_{2}$ express the red pigment. This apparently simplistic model was later confirmed by experimental observations on the early Drosophila embryo, where the concentration gradient of the protein Bicoid (i.e. a transcription factor, being the first molecules found to act as a morphogen in 1988 [36]) drives an antero-posterior differentiation in three separated domains [69].

The models proposed by Turing and Wolpert offer two different points of view on pattern formation. A first difference comes from the fact that Turing aimed at modeling spontaneous formation of a pattern, while Wolpert asked how a more complex pattern can arise from an asymmetry (i.e. polarity) within the tissue. Furthermore, in Turing's model the concentration of morphogens is directly related to the spatial pattern, in this sense it is a pre-pattern. Conversely, Wolpert introduced an interpretation step where the cell activity is specified by the concentration gradient. Moreover, Wolpert himself later argued that morphogenetic movements are rather determined by other transport mechanisms than passive diffusion, such as pla- 


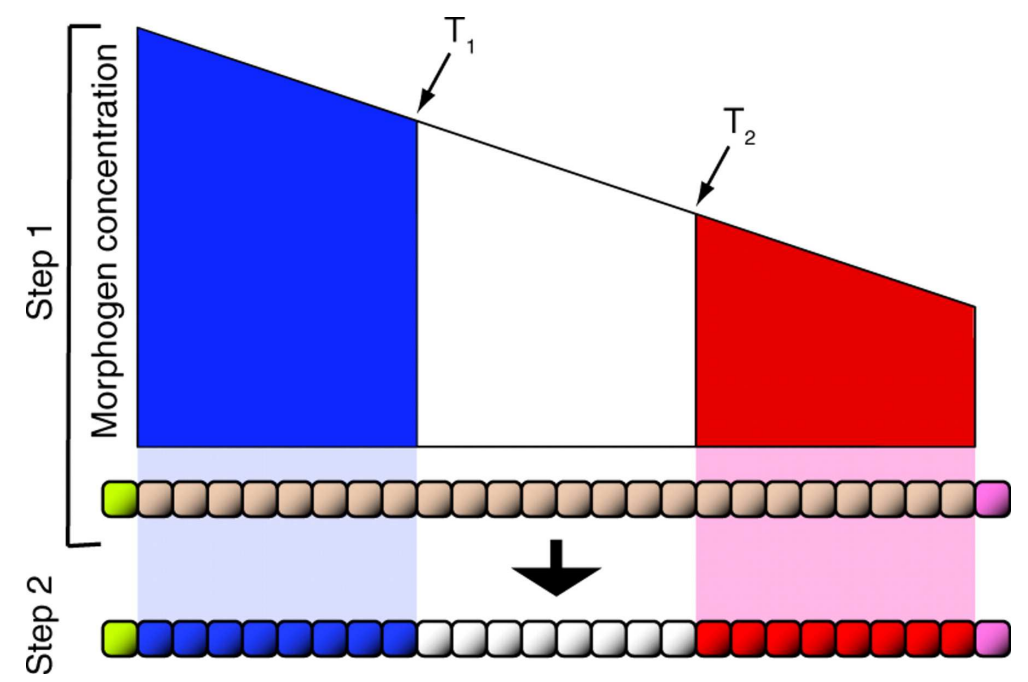

Fig. 3 The French Flag model: positional information is specified by the gradient of a morphogen concentration. Adapted from [68].

nar transcytosis and cytonemes [120]. Moreover, morphogens should be generally subjected to fluctuations on short length-scales whilst travelling through individual cells, yet finally delivering precise positional information. Without discussing in further details such limiting aspects, it is now generally accepted that more complicated morphogenetic models are needed in the wide scenario of embryogenesis. For example, recent works have proposed a different mechanism through which the two models can cooperate in pattern formation [52].

\subsubsection{The new course of genetics and the return of an ancient dichotomy}

Although the origins of genetics date back to the second half of the 19th Century, when Gregor Mendel discovered the inheritance of biological traits [88], its fundamental role in morphogenesis finally emerged only during the 20th Century [121]. Indeed, the earlier vision of genetics uniquely concerned studying the transmission of hereditary properties in the embryo, since Mendel's theory was based on the idea that the hereditary package is transmitted from parents to offspring through a set of discrete hereditary factors. Although proved by experimenting on pea plants, Mendel's theory was unable to provide any concrete description of the nature of such factors. A breakthrough occurred with the discovery that the DNA carries the genetic information and that genes encode proteins. Thus, the properties of a cell are determined by the proteins they contain, genes control and act on proteins in order to drive the cell fate and consequently, the development of a shape. Right after 
these discoveries, a lot of experiments focused on finding the genes responsible for several morphogenetic events in the embryo. The strategy adopted was to introduce changes in the molecular organization of the DNA in order to observe abnormal changes in a final structure. In this way, it has been possible to identify which genes play an active role in the formation of an emerging pattern.

These novel discoveries puzzled researchers about the genetic bases of morphogenesis. Interestingly, the ancient dichotomy between pre-formationism and epigenesis rephrased in a modern key, whether the the shape of an organism emerges as a results of a set of fixed genetic instructions or it can be influenced by environmental factors, both at the individual level and during evolution.

Even if many open questions exist in the rapidly developing research branches of morphogenesis, it is now acknowledged that both the genetic and the epigenetic factors influence shape at different levels [7]. In the absence of mutations, each cell within a given organism has the same set of genes, called genotype, whilst only a small subset is expressed as a function of the environment, defining the phenotype. Therefore, the epigenetic adaptation can occur both at the level of individual organism as a change of phenotype and at the evolutionary level as a switch of ontogenic programs [2]. In summary, it is now clear that the morphogenetic processes (e.g. cell division and differentiation) are driven by genetic signals, as well as that the genetic expression involves epigenetic processes by means of morpho-regulatory biomolecules, that interact with genes through a complex network of feedback regulatory mechanisms [38].

\subsection{The open quest for the chemo-mechanical cues of morphogenesis}

Nowadays, there is an increasing research interest in life sciences concerning the biomolecular mechanisms directing the adaptation of living matter to the physical forces exerted by the surrounding micro-environment. The ensemble of biologically processes converting mechanical forces into biochemical factors is called mechanotransduction. This is a typical multi-scale phenomenon, since living cells may sense from nano-scale to macroscopic forces often driving the transition from physiological to pathological conditions [37]. The nano-scale force transduction occurs by means of cell membrane molecules, typically focal adhesion proteins and integrins, converting the mechanical stimulus into a change of membrane potential through the formation of a ionic current. In physiological condition, a feedback mechanism, known as mechano-reciprocity, acts for activating the acto-myosin machinery in order to restore a tensional homeostasis at the macro-scale (e.g. tissue), i.e. globally balancing endogenous and exogenous forces. Conversely, a loss of tensional homeostasis often characterizes a hallmark of disease, e.g. directing both the initiation of solid tumours and the formation of metastasis [15].

Mechano-transduction plays a fundamental role in the modern view of morphogenesis, since it allows the regulation of epigenetic and genomic factors at different 
time-scales. In fact, it control the dynamic adaptation of living cells by means of both switch-like events at their trans-membrane structures [122] and the activation dynamic molecular processes in response to long time-varying mechanical stimuli [61]. Many seminal experiments highlighting the crucial role of chemo-mechanical cue in morphogenesis have been performed in the last decades by the research group lead by Lev Beloussov. In particular, these works have reported that the active responses of living matter may overshoot the passive mechanical stimuli, proposing a hyper-restoration principle for homeostasis [8]. Another open area of investigation concerns the identification of the local regulation mechanisms, mainly signalling pathways that spatially and temporally control cell behaviour, which allow the global orchestration of a macroscopic shape [76]. Therefore, the current understanding of morphogenetic events gained a further complexity in the underlying phenomena with respect to the purely chemical bases earlier assumed. For example, the local concentration of morphogens can trigger growth as a random cellular proliferation. Nonetheless, polarity can induce preferred orientations in cellular division, possibly affected also both by competition between different cell populations and by physical forces, e.g. growth inhibition by compression [78]. At a more macroscopic level, unknown chemo-mechanical orchestration mechanisms should transform random or oriented local proliferations into a uniform growth, which must ultimately cease as the final size is reached. Thus, the morphological control may depend on both the cell number and the overall size, suggesting that the spatial gradient of morphogens can provide cells a dimension-sensing mechanism.

The quest for the chemo-mechanical cues in morphogenesis is therefore a mandatory step for the development of refined mathematical theories [66]. This was somewhat hinted by Turing himself in his original paper:

In the continuous form of the theory [..] one should take into account

(i) The changes of position and velocity as given by Newton's laws of motion.

(ii) The stresses as given by the elasticities and motions[...].

(iii) The chemical reactions.

(iv) The diffusion of the chemical substances[...].

This account of the problem omits many features, [...] but even so it is a problem of

formidable mathematical complexity. The interdependence of the chemical and mechanical data adds enormously to the difficulty [...].

Well aware of such unavoidable complications, we will sketch in the next section the balance laws for a continuous theory of morphogenesis, using the thermodynamics of open systems to take into account for the chemo-mechanical coupling.

\section{A continous chemo-mechanical theory of morphogenesis}

The thermo-mechanics of open systems is a natural framework for a macroscopic mathematical description of morphogenesis in living matter, which is hereafter considered as a continuous distribution of matter. Accordingly, we disregard the micro- 
scopic dynamics of the underlying biological phenomena acting at the subcellular or cellular level, and we model the relevant chemo-mechanical properties by averaged fields at the tissue scale. In the following, we provide some basic kinematic notions and we derive the physical balance laws that govern the morphogenetic processes within living matter as a function of both the mechanical and the chemical cues.

\subsection{Basic kinematic notions}

Denoting by $\mathscr{E} \subset \mathbb{R}^{3}$ be a three-dimensional Euclidean space, let $\mathscr{B}_{0}, \mathscr{B}_{a} \subset \mathscr{E}$ be two regions occupied by the living body in two different instants of time. Let $\chi$ be the mapping which transforms the tissue from its initial configuration $\mathscr{B}_{0}$ to its final configuration $\mathscr{B}_{a}$, which can be defined as the following $\mathscr{C}^{1}$-diffeomorphism:

$$
\chi: \mathscr{B}_{0} \rightarrow \mathscr{B}_{a} \quad \mathbf{x}=\chi(\mathbf{X}), \quad \text { with inverse } \quad \mathbf{X}=\chi^{-1}(\mathbf{x})
$$

where $\mathbf{X}, \mathbf{x}$ are the position vectors in the reference and actual configurations $\mathscr{B}_{0}$ and $\mathscr{B}_{a}$, respectively. The deformation gradient, associated to the deformation in Eq.(1), is the second order two-point tensor defined as:

$$
\mathbf{F}: T \mathscr{B}_{0} \rightarrow T \mathscr{B}_{a} \quad \mathbf{F}=\operatorname{Grad} \mathbf{x}=\frac{\partial \chi(\mathbf{X})}{\partial \mathbf{X}}=\frac{\partial x_{i}}{\partial X_{j}} \mathbf{e}_{i} \otimes \mathbf{E}_{j}
$$

where $\mathbf{E}_{j}, \mathbf{e}_{i}$ (with $i, j \in\{1,2,3\}$ ) are the basis unit vectors in the reference and actual configurations, respectively, and $T \mathscr{B}_{0}, T \mathscr{B}_{a}$ are the collections of all tangent spaces on $\mathscr{B}_{0}$ and $\mathscr{B}_{a}$, respectively. A tangent space is the set of all line elements attached to a body point. The capital notation Grad in Eq.(2) indicates the gradient operator with respect to the material position $X_{j}$ in the reference configuration $\mathscr{B}_{0}$ and the symbol $\otimes$ indicates the dyadic product between two vectors, in indicial form $:(\mathbf{a} \otimes \mathbf{b})_{\alpha \beta}=a_{\alpha} b_{\beta}$.

According to Eq. (2), the following relations for the transformation of line, surface and volume elements, respectively, hold:

$$
\begin{aligned}
& \mathbf{d x}=\mathbf{F d} \mathbf{X} \\
& \mathbf{n d s}=J \mathbf{F}^{-T} \mathbf{N d S} \\
& \mathrm{d} v=J \mathrm{~d} V
\end{aligned}
$$

where $J=\operatorname{det} \mathbf{F}$ and the quantities $\mathbf{d X}, \mathbf{N d S}, \mathrm{d} V$ and $\mathbf{d x}, \mathbf{n d s}, \mathrm{d} v$ are defined in the reference and actual configurations, respectively, with $\mathbf{N}$ and $\mathbf{n}$ being the unit normal vectors to dS and ds, respectively. Eq. (5) can be directly derived from Eq. (3), while Eq (4) is also known as Nanson's Formula.

Before deriving the thermo-mechanical laws for a morphogenetic process, it is useful to introduce some relations that will be used in the following.

Let $\mathbf{f}(\mathbf{x}, t)$ be a continuously differentiable spatial vector field, its total time deriva- 
tive writes as:

$$
\dot{\mathbf{f}}(\mathbf{x}, t)=\frac{d \mathbf{f}(\mathbf{x}, t)}{d t}=\frac{\partial \mathbf{f}(\mathbf{x}, t)}{\partial t}+\mathbf{v}(\mathbf{x}, t) \operatorname{grad} \mathbf{f}(\mathbf{x}, t),
$$

Moreover, considering the integral of the spatial field $\mathbf{f}$ on a volume changing with time, the Reynolds theorem allows for the time derivative to be calculated as:

$$
\frac{d}{d t} \int_{\mathscr{B}_{a}} \mathbf{f}(\mathbf{x}, t) \mathrm{d} v=\int_{\mathscr{B}_{a}}[\dot{\mathbf{f}}(\mathbf{x}, t)+\mathbf{f}(\mathbf{x}, t) \operatorname{div} \mathbf{v}(\mathbf{x}, t)] \mathrm{d} v,
$$

where $\mathbf{v}(\mathbf{x}, t)$ is the spatial velocity, and div is the spatial divergence operator. Moreover, we will use the divergence theorem in the form :

$$
\int_{\partial \mathscr{B}_{a}} \mathbf{n} \cdot \mathbf{f}(\mathbf{x}, t) \mathrm{d} s=\int_{\mathscr{B}_{a}} \operatorname{div} \mathbf{f}(\mathbf{x}, t) \mathrm{d} v
$$

so that $\operatorname{div}(\mathbf{f})=\mathrm{f}_{i h, i}$ for any given tensor field $\mathbf{f}$, assuming saturation on repeated indices unless explicitly stated.

\subsubsection{Balance of mass}

Since morphogenetic processes usually involve growth and remodelling, let us first derive the mass balance for a living material as a function of the biochemical factors $[40,26]$. The global form of the mass balance for a growing body $\mathrm{X}$ in spatial coordinates reads:

$$
\frac{d}{d t} \int_{\mathscr{B}_{a}} \rho \mathrm{d} v=\int_{\mathscr{B}_{a}} \omega \mathrm{d} v
$$

where $\rho$ is the spatial mass density and $d / d t$ denotes the total time material derivative. Accordingly, $\omega$ is the internal mass production rate per unit current volume, and we neglected any non-convective mass flux for the sake of brevity. The interested reader is referred to [39] for the introduction of mass self-diffusion terms.

The mass production rate represents the cellular proliferation within the material which is possibly driven by some biochemical fields, e.g. growth signals, nutrients, morphogens. Indicating with $c_{i}(\mathbf{x}, t)$ the concentration of the generic $i-t h$ species per unit volume, we can generically assume that $\omega=\omega\left(c_{i}, \mathbf{F}\right)$. Moreover, since we deal with signals or low weight molecules, such species can me modeled as internal variables with negligible inertia [21], having the following balance laws:

$$
\frac{d}{d t} c_{i}-\operatorname{div} \mathbf{J}_{c i}=-\xi_{i}(\mathbf{F}, \operatorname{Grad} \mathbf{F})
$$

where $\xi_{i}$ is the absorption rate of the $i-t h$ species, whose value may depend both on the first and on the second gradient of the deformation field, the latter reproducing, for example, curvature-dependent effects in the absorption of angiogenetic factors [80]. The diffusion of a morphogen from the source through the extracellular matter, 
with a sink function possibly regulated by receptor endocytosis, has been proved to describe the morphogens gradient in multicellular embryonic tissues [123]. As discussed in [4], the simpler expression for the flux of nutrients $\mathbf{J}_{c}$ is given:

$$
\mathbf{J}_{c i}=D\left(c_{i}\right) J \operatorname{grad} c_{i}
$$

where $D\left(c_{i}\right)$ represents a diffusion tensor, whose positive definiteness and monotonicity on $c_{i}$ ensure the stability of the solution.

The local spatial form of Eq.(51), can now be easily derived by substituting Eq.(7)and permuting integration and differentiation, being:

$$
\dot{\rho}+\rho \operatorname{div} \mathbf{v}=\omega\left(c_{i}, \mathbf{F}\right),
$$

where the dot symbol indicates the total time derivative defined in Eq.(6). For problems involving solids, it is useful to derive its material counterpart using a similar procedure after recasting Eq.(51) in the reference volume. It reads :

$$
\dot{\rho}_{0}=\Omega
$$

where $\rho_{0}=J \rho$ is the material density and $\Omega=J \omega$ is the internal mass production rate per unit reference volume.

In summary, Eqs. $(12,13)$ are the mass balance laws of biological material during a morphogenetic process guided by the reaction diffusion Eq.(10). We remark that the driving chemo-mechanical cues will be defined by the proper constitutive choices of the coupling terms $\omega$ and $\xi$.

\subsection{Balance of linear and angular momentum}

Let us first consider the balance of the physical linear momentum $\mathbf{p}=\rho \mathbf{v}$ inside the biological system. Its conservation law in the global form rewrites:

$$
\frac{d}{d t}\left(\int_{\mathscr{B}_{a}} \mathbf{p} d v\right)=\int_{\mathscr{B}_{a}}\left(\rho \mathbf{b}_{v}+\omega \mathbf{v}\right) d v+\int_{\partial_{\mathscr{B}_{a}}} \mathbf{n} \cdot \sigma d s
$$

where $\sigma$ is the Cauchy stress tensors, and $\mathbf{b}_{v}$ is an external bulk force. Substituting Eq.(12) while developing the integral terms in Eq.(14), we obtain after some standard manipulations the following local form in the spatial configuration:

$$
\rho \frac{d \mathbf{v}}{d t}=\rho \mathbf{b}_{v}+\operatorname{div} \sigma
$$

In order to derive the material counterpart of Eq.(14), let us substitute the Nanson's formula in Eq.(4), so that:

$$
\frac{D}{D t} \int_{\mathscr{B}_{0}} \rho_{0} \mathbf{v} \mathrm{dV}=\int_{\partial \mathscr{B}_{0}} \mathbf{N} . \mathbf{S} \mathrm{d} \mathbf{S}+\int_{\mathscr{B}_{0}}\left(\rho_{0} \mathbf{b}_{v}+\omega \mathbf{v}\right) \mathrm{dV}
$$


where the volume element transformation in Eq.(5) and the relation in Eq.(12) have been used. The stress tensor $\mathbf{S}$ in Eq.(16) is called the Nominal stress and is related to the Cauchy stress by $\mathbf{S}=J \mathbf{F}^{-1} \sigma^{3}$. Thus, its local form in material coordinates reads:

$$
\rho_{0} \frac{d \mathbf{v}}{d t}=\rho_{o} \mathbf{b}_{v}+\operatorname{Div} \mathbf{S}
$$

where Div is the material divergence.

Furthermore, the global form of the angular momentum balance in presence of growth can be written in spatial coordinates as:

$$
\frac{D}{D t} \int_{\mathscr{B}_{a}} \mathbf{y} \times \rho \mathbf{v} \mathrm{d} v=\int_{\partial \mathscr{B}_{a}} \mathbf{y} \times \sigma \mathbf{n} \mathrm{ds}+\int_{\mathscr{B}_{a}} \mathbf{y} \times\left(\rho \mathbf{b}_{v}+\omega \mathbf{v}\right) \mathrm{d} v
$$

where $\times$ is the cross product and the position vector $\mathbf{y}$ has been introduced as $\mathbf{y}=$ $\mathbf{x}-\mathbf{x}_{0}$ with $\mathbf{x}_{0}$ being a fixed vector position. Using the Reynolds theorem in Eq.(7), the 1.h.s of Eq.(18), rewrites:

$$
\frac{D}{D t} \int_{\mathscr{B}_{a}} \mathbf{y} \times \rho \mathbf{v} \mathrm{d} v=\int_{\mathscr{B}_{a}}[\mathbf{y} \times(\dot{\rho} \mathbf{v}+\rho \dot{\mathbf{v}})+(\mathbf{y} \times \rho \mathbf{v}) \operatorname{div} \mathbf{v}] \mathrm{d} v
$$

where the second equality follows from $\dot{\mathbf{y}}=\dot{\mathbf{x}}=\mathbf{v}$ and the product differentiation rule of the cross product.

Using the divergence theorem in Eq.(8) and the properties of the cross product, the first term in the r.h.s of Eq.(18) transforms into the volume integral:

$$
\int_{\partial \mathscr{B}_{a}} \mathbf{y} \times \sigma \mathbf{n d s}=\int_{\mathscr{B}_{a}}\left[\mathbf{y} \times \operatorname{div} \sigma+\mathscr{E}: \sigma^{T}\right] \mathrm{dV}
$$

where $\mathscr{E}=\varepsilon_{j k l} \mathbf{e}_{j} \otimes \mathbf{e}_{k} \otimes \mathbf{e}_{l}$ is the third-order permutation tensor with components $\varepsilon_{j k l}=\left(\mathbf{e}_{j} \times \mathbf{e}_{k}\right) \cdot \mathbf{e}_{l}$. Now, using the mass balance in Eq.(12), the balance of linear momentum in Eq.(15) and substituting Eqs.(19,20) into Eq.(18), it follows that:

$$
\int_{\mathscr{B}_{a}} \mathscr{E}: \sigma^{T} \mathrm{dV}=\mathbf{0}
$$

whose local eulerian form is:

$$
\sigma=\sigma^{T}
$$

with material counterpart:

$$
\mathbf{F S}=\mathbf{S}^{T} \mathbf{F}^{T}
$$

Hence, the Cauchy stress tensor $\sigma$ must be symmetric whilst the Nominal stress $\mathbf{S}$ is not. In the following, we will derive the thermodynamic principles for the growing biological system.

\footnotetext{
${ }^{3}$ Since $\mathbf{S}$ is related to $\sigma$ through a Piola transformation, it is often called first Piola-Kirchooff stress tensor [87]. Nonetheless, some authors consider the latter being the transpose of $\mathbf{S}$ [91]. Accordingly we prefer to call it nominal stress in order to avoid misunderstanding to the readers
} 


\subsection{Balance of internal energy and entropy inequality}

Following the approach in [40], let us indicate with $\varepsilon$ the internal energy per unit mass and with $\mu_{i}$ a scalar function representing the source of internal energy associated with the $i-t h$ solute $c_{i}$. Accordingly, the global form of the first law of thermodynamics can be expressed as follows:

$$
\frac{d}{d t} \int_{\mathscr{B}_{a}} \rho \varepsilon d v=\int_{\mathscr{B}_{a}}\left(\omega \varepsilon-\xi_{i} c_{i} \mu_{i}+r_{0}+\operatorname{tr}(\sigma \mathbf{d})\right) d v+\int_{\partial \mathscr{B}_{a}} \mathbf{n} \cdot\left(\mu_{i} \mathbf{J}_{c i}-\mathbf{Q}\right) d s
$$

were $\mathbf{d}=\left(\operatorname{grad} \mathbf{v}+(\operatorname{grad} \mathbf{v})^{T}\right) / 2, r_{0}$ is the external heat supply per unit of volume, $\mathbf{Q}$ is the heat flux, $\mu_{i}$ is the chemical potential of the $\mathrm{i}$-th species and represents the increase of internal energy of the continuum by physical absorption of the diffusing chemicals. Irreversible terms and a temperature gradient dependence have been neglected for the sake of simplicity. Substituting Eqs.(10,12, 15) in Eq.(24), we obtain the following local form for the balance of the internal energy in a growing material:

$$
\rho \dot{\varepsilon}=\operatorname{tr}(\sigma \mathbf{d})+\mu_{i} \dot{c}_{i}-\operatorname{div} \mathbf{Q}+r_{0}+\mathbf{J}_{c i} \cdot \operatorname{grad} \mu_{i}
$$

The global form of Clausius-Duhem entropy inequality reads:

$$
\frac{d}{d t} \int_{\mathscr{B}_{a}} \rho_{0} \eta d v \geq \int_{\mathscr{B}_{a}}\left(\omega \eta-\eta_{i} \xi_{i} \dot{c}_{i}+\frac{r_{0}}{\Theta}\right) d v+\int_{\partial \mathscr{B}_{a}} \mathbf{n} \cdot\left(\mathbf{J}_{c i} \eta_{i}-\frac{\mathbf{Q}}{\Theta}\right) d s
$$

where $\eta, \eta_{i}$ are the entropy density per unit mass and per unit of solvent concentration, respectively. The local form of the entropy inequality, substituting Eq.(12) in Eq.(26), reads:

$$
\rho \dot{\eta} \geq \eta_{i} \dot{c}_{i}+\mathbf{J}_{c i} \cdot \operatorname{grad} \eta_{i}+\frac{r_{0}}{\Theta}-\operatorname{div}\left(\frac{\mathbf{Q}}{\Theta}\right)
$$

Recalling the expression of the Helmholtz free energy per unit of mass, $\Psi=(\varepsilon-$ $\Theta \eta)$, and per unit of solvent concentration, $\Psi_{i}=\left(\mu_{i}-\Theta \eta_{i}\right)$, we can put together Eqs. $(25,27)$ in order to obtain an equivalent form of the entropy inequality:

$$
\rho(\dot{\Psi}+\dot{\Theta} \eta) \leq \operatorname{tr}(\sigma \mathbf{d})+\Psi_{i} \dot{c}_{i}+\mathbf{J}_{c i} \cdot\left(\nabla_{R} \Psi_{i}+\eta_{i} \nabla_{R} \Theta\right)-\frac{\mathbf{Q}}{\Theta} \cdot \nabla_{R} \Theta
$$

The latter inequality describes the thermodynamical consistency for the energy dissipation during a morphogenetic process, accounting both for mass production and for the reaction-diffusion of the biochemical species. Once defined the constitutive equation for the stresses, Eq.(28) allows defining a suitable chemo-mechanical coupling regulating the evolution of the biological system. An extension of the presented thermo-mechanic framework to strain gradient continua can be found in [22]. 


\subsection{Balance laws for interfacial morphogenetic processes}

Morphogenetic processes can occur at different scales, which are governed by the typical lengths characterizing the local interplay between mass production and the reaction- diffusion properties of biomolecules. For example, cells duplicate inside narrow regions created by the diffusion fronts of morphogenetic signals, whilst a macroscopic remodelling occurs in order to reach an homeostatic state at the tissue level [77]. It is therefore useful to extend the balance laws derived in the previous paragraphs to the case in which the bulk mass changes are localised into a small volume. For the sake of clarity, let us consider a biological system made by two different materials occupying growing adjacent regions $V^{-}(t)$ and $V^{+}(t)$, separated by the moving surface $\Sigma(t)$ with outer normal $\mathbf{n}_{\Sigma}$. Such surface will be considered as a non-material interface [28], i.e. carrying thermo-mechanical properties, and described using a local parametrization expressing its spatial position as $\mathbf{x}=\mathbf{x}\left(u^{1}, u^{2}\right)$, with tangent bases $\mathbf{a}_{l}=\mathbf{x}_{, u^{l}}$ with $l=(1,2)$. The parametric velocity $\overline{\mathbf{v}}_{\Sigma}$ of the surface can be decomposed as:

$$
\overline{\mathbf{v}}_{\Sigma}=\overline{\mathbf{v}}_{\Sigma s}+\bar{v}_{\Sigma n} \mathbf{n}_{\Sigma}
$$

where $\overline{\mathbf{v}}_{\Sigma s}$ is assumed to correspond to the projection of the physical velocity on the surface, whose value depends on the parametrization. Moreover, every a point on $\Sigma(t)$ has a physical velocity $\mathbf{v}_{\Sigma}$, and we assume the compatibility condition $v_{\Sigma s}=\bar{v}_{\Sigma s}$. In the following the superscripts - and + will be used for indicating the physical fields inside the volumes having outer normal $\mathbf{n}^{-}=-\mathbf{n}^{+}=\mathbf{n}_{\Sigma}$, respectively. The volumetric physical fields must obey the balance principles previously derived.

Morphogenetic processes occur in a very narrow layer of thickness $\varepsilon$, defined as:

$$
\Delta V_{\varepsilon}=\bigcup\left(\mathbf{x}+v \mathbf{n}_{\Sigma}\right) ; \forall \mathbf{x} \in \Sigma(t),-\varepsilon / 2 \leq v \leq \varepsilon / 2
$$

so that we can obtain surface fields on $\Sigma(t)$ by homogenization of the volume fields, calculating their finite limit for $\varepsilon \rightarrow 0$. Indicating with the subscript $\Sigma$ such interfacial physical fields, we can therefore derive the mechanical balance laws and write the thermo-dynamic principles for the entire system. Dealing with a moving discontinuity, we define the Thomas derivative as $\frac{\delta_{t}(\cdot)}{\delta_{t} t}=\frac{\partial(\cdot)}{\partial t}+\bar{v}_{\Sigma n} \mathbf{n}_{\Sigma} \cdot \nabla(\cdot)$, and we introduce the jump operator $\llbracket \cdot \rrbracket=(\cdot)^{+}-(\cdot)^{-}$.

For matters of generality, we assume that generic mass fluxes $\mathbf{m}^{-}$and $\mathbf{m}^{+}$may exist between the volumes and the moving interface ${ }^{4}$. Therefore, by applying the transport and divergence theorems in a system with a non-material discontinuity [99], the surface mass balance takes the following form:

$$
\frac{\delta_{t} \rho_{\Sigma}}{\delta_{t} t}+\operatorname{div}_{\Sigma} \cdot\left(\rho_{\Sigma} \mathbf{v}_{\Sigma s}\right)-K \rho_{\Sigma} \bar{v}_{\Sigma n}=\rho_{\Sigma} \omega_{\Sigma}+\llbracket \rho\left(\bar{v}_{\Sigma n}-v_{n}\right)+\mathbf{n}_{\Sigma} \cdot \mathbf{m} \rrbracket
$$

\footnotetext{
${ }^{4}$ We remark that for Galilean invariance they should be somehow dependent on a relative velocity
} field. 
where $\operatorname{div}_{\Sigma} \cdot$ is the surface divergence, $K$ is twice the local mean curvature, $v_{n}$ is the normal component of the physical velocity and $\omega_{\Sigma}$ is the surface mass source.

Using eq.(31) and neglecting surface external forces, the balance of linear momentum on $\Sigma(t)$ reads:

$$
\begin{aligned}
\rho_{\Sigma} & \frac{\delta_{i} \mathbf{v}_{\Sigma}}{\delta_{t} t}+\left(\rho_{\Sigma} \mathbf{v}_{\Sigma s} \cdot \operatorname{grad}_{\Sigma}\right) \mathbf{v}_{\Sigma}=\operatorname{div}_{\Sigma} \cdot \sigma_{\Sigma}+ \\
& \llbracket\left(\mathbf{v}-\mathbf{v}_{\Sigma}\right)\left(\rho\left(\bar{v}_{\Sigma n}-v_{n}\right)+\mathbf{n}_{\Sigma} \cdot \mathbf{m}\right)+\mathbf{n}_{\Sigma} \cdot \sigma \rrbracket
\end{aligned}
$$

where $\sigma_{\Sigma}$ is the Cauchy stress tensor acting on the surface. We remark that eq.(32) generalizes the Young-Laplace law for a growing non-material interface.

The balance of angular momentum on the surface reads:

$$
\mathbf{a}^{l} \cdot \sigma_{\Sigma} \times \mathbf{a}_{l}=0 \quad l=(1,2)
$$

where $\times$ is the cross-product and $\mathbf{a}^{l}$ indicate the reciprocal tangent bases. Thus, $\sigma_{\Sigma}$ must be a tangential field on $\Sigma(t)$ with symmetric surface components.

The first and second laws of thermodynamics after standard manipulations rewrite:

$$
\begin{aligned}
& \rho_{\Sigma} \dot{\varepsilon}_{\Sigma}=\mathbf{a}^{l} \cdot \sigma_{\Sigma} \cdot \operatorname{grad}_{\Sigma} \mathbf{v}_{\Sigma}-\operatorname{div}_{\Sigma} \cdot \mathbf{Q}_{\Sigma}+r_{\Sigma} \\
& +\llbracket \mathbf{n}_{\Sigma} \cdot \sigma \cdot\left(\mathbf{v}-\mathbf{v}_{\Sigma}\right)+\left(\frac{\left(\mathbf{v}-\mathbf{v}_{\Sigma}\right)^{2}}{2}+\varepsilon-\varepsilon_{\Sigma}\right)\left(\rho\left(\bar{v}_{\Sigma n}-v_{n}\right)+\mathbf{n}_{\Sigma} \cdot \mathbf{m}\right)-\mathbf{n}_{\Sigma} \cdot \mathbf{Q} \rrbracket \\
& \rho_{\Sigma} \dot{\eta}_{\Sigma} \geq \frac{r_{\Sigma}}{\Theta}-\nabla \cdot\left(\frac{\mathbf{Q}_{\Sigma}}{\Theta}\right)-\llbracket \mathbf{n}_{\Sigma} \cdot\left(\frac{\mathbf{Q}}{\Theta}+\overline{\mathbf{Q}}\right)-\left(\eta-\eta_{\Sigma}\right)\left(\rho\left(\bar{v}_{\Sigma n}-v_{n}\right)+\mathbf{n}_{\Sigma} \cdot \mathbf{m}\right) \rrbracket
\end{aligned}
$$

where $\overline{\mathbf{Q}}$ represents a bulk extra-entropy flux, whose jump may represent the transfer of biochemical energy within the interface. Putting together eqs. $(34,35)$ and defining a surface free energy $\Psi_{\Sigma}$ per unit mass, the dissipation inequality on the surface can be written as:

$$
\begin{aligned}
& \rho_{\Sigma} \dot{\Psi}_{\Sigma} \leq \mathbf{a}^{l} \cdot \sigma_{\Sigma} \cdot \mathbf{v}_{\Sigma, l}+\llbracket \mathbf{n}_{\Sigma} \cdot \sigma \cdot\left(\mathbf{v}-\mathbf{v}_{\Sigma}\right)-\Theta \mathbf{n}_{\Sigma} \cdot \overline{\mathbf{Q}} \\
& +\left(\frac{\left(\mathbf{v}-\mathbf{v}_{\Sigma}\right)^{2}}{2}+\Psi-\Psi_{\Sigma}\right)\left(\rho\left(\bar{v}_{\Sigma n}-v_{n}\right)+\mathbf{n}_{\Sigma} \cdot \mathbf{m}\right) \rrbracket
\end{aligned}
$$

where we assumed isothermal, uniform conditions for matters of notation compactness. In particular the quantity between the bracket operator represents the flux of the chemo-mechanical energy flux vector, i.e. a generalization of the Poynting vector for the moving non-material interface. In summary, once defined the constitutive equation for the surface stress, the dissipation inequality in eq.(36) allows defining thermodynamically consistent evolution laws for the interfacial morphogenesis.

An application: morphogenesis of seashells Let us consider the proposed thermomechanical model of interfacial growth to describe the morphogenesis of seashells [27]. As accurately investigated by D'Arcy Thompson [112], seashells also display a great variety of shapes and patterns, often characterized by a self-similar geometric structure, following a logarithmic (equiangular) helico-spiral. 
The growing shell is generated by a tongue-like protrusion of the mollusc, known as the mantle, which wraps around the grown shell edge for depositing both new material and pigments. The surface growth process here occurs at a moving surface $\Sigma(t)$ at the edge of the shells, and the secretion velocity is found to be controlled by the neural system of the mantle [12]. Indeed, the position $\rho(\theta)=\sqrt{x^{2}+y^{2}+z^{2}}$ of the mantle can be expressed in a logarithmic helico-spiral as a function of the turning angle $\theta$, reading:

$$
\left\{\begin{array}{l}
x=\rho_{0} \sin \beta \cos \theta e^{\theta \cot \alpha} \\
y=\rho_{0} \sin \beta \sin \theta e^{\theta \cot \alpha} \\
z=\rho_{0} \cos \beta e^{\theta \cot \alpha}
\end{array}\right.
$$

where $\beta$ is the enlarging angle and $\alpha$ the wrapping (equiangular) angle of the spiral, as shown in Figure 4(a). In practice, if $R=\sqrt{x^{2}+y^{2}}$, the components of the mantle velocity are linked by the following relation: $\dot{z} \tan \beta=\dot{R}$.

Considering a moving reference framework whose origin represents the mantle position, the deposition of new material is modeled to occur on a generating curve on the moving surface $\Sigma(t)$, which has unit vectors $\mathbf{e}_{z}$ and $\mathbf{e}_{\rho}=\mathbf{e}_{x} \cos \theta+\mathbf{e}_{y} \sin \theta$. The volumetric mass balance equation in Eq.(??) for the shell imposes:

$$
\rho^{-} \operatorname{div} \mathbf{v}^{-}=0
$$

where $\rho^{-}$is the constant volume density of the fabricated material. Choosing a generating circular section for the sake of simplicity, its outer radius $r(\theta)$ can be given on a polar parametric representation $(r, s)$ on $\Sigma(t)$. If the mantle deposit a surface mass source $\gamma_{\Sigma}$, a solution of the the surface mass balance in Eq.(??) is given by:

$$
\mathbf{m}^{-}=\left[\rho^{-}\left(\mathbf{v}^{-}-\overline{\mathbf{v}}_{\Sigma}\right) \cdot \mathbf{e}_{n}\right] \mathbf{e}_{n} ; \quad \operatorname{div}_{\Sigma} \cdot \overline{\mathbf{v}}_{\Sigma s}=\gamma_{\Sigma} \quad \Rightarrow \quad r(t)=r(0) e^{\frac{\gamma_{\Sigma} t}{2}}
$$

with $\mathbf{e}_{n}=\mathbf{e}_{z} \wedge \mathbf{e}_{\rho}$ being the normal unit vector of the growing surface, setting $\bar{v}_{\Sigma n}=\left(\overline{\mathbf{v}}_{\Sigma} \cdot \mathbf{e}_{n}\right)=\rho(\theta) \dot{\theta}$ in virtue of Eq.(37). Moreover, the parametrization is chosen such that the $\Sigma(t)$ moves with the center of mass of the growing mass, i.e. $\bar{v}_{\Sigma n}=v_{\Sigma n}$. Under these assumptions, Eq.(36) ensures that the seashell growth is thermodynamically consistent.

The shell morphology depends on the combination of the helico-spiral movement of the mantle, through the angles $\alpha, \beta$ in Eq.(37), and the displacement of the edge radius, given by the surface mass source $\gamma_{\Sigma}$ in Eq.(39). Assuming that the spiral velocity of the mantle is constant, so that $\dot{\theta}=m$, we can impose that the growing material is always deposited tangentially to the shell, reading:

$$
\begin{aligned}
& \rho(\theta+2 \pi)-\rho(\theta)=r\left(\frac{\theta+2 \pi}{m}\right)+r\left(\frac{\theta}{m}\right) ; \\
& \rho_{0} e^{\theta \cot \alpha}\left(e^{2 \pi \cot \alpha}-1\right)=r(0) e^{\frac{\gamma_{\Sigma} \theta}{2 k}}\left(e^{\frac{\gamma_{\Sigma} \pi}{m}}+1\right)
\end{aligned}
$$



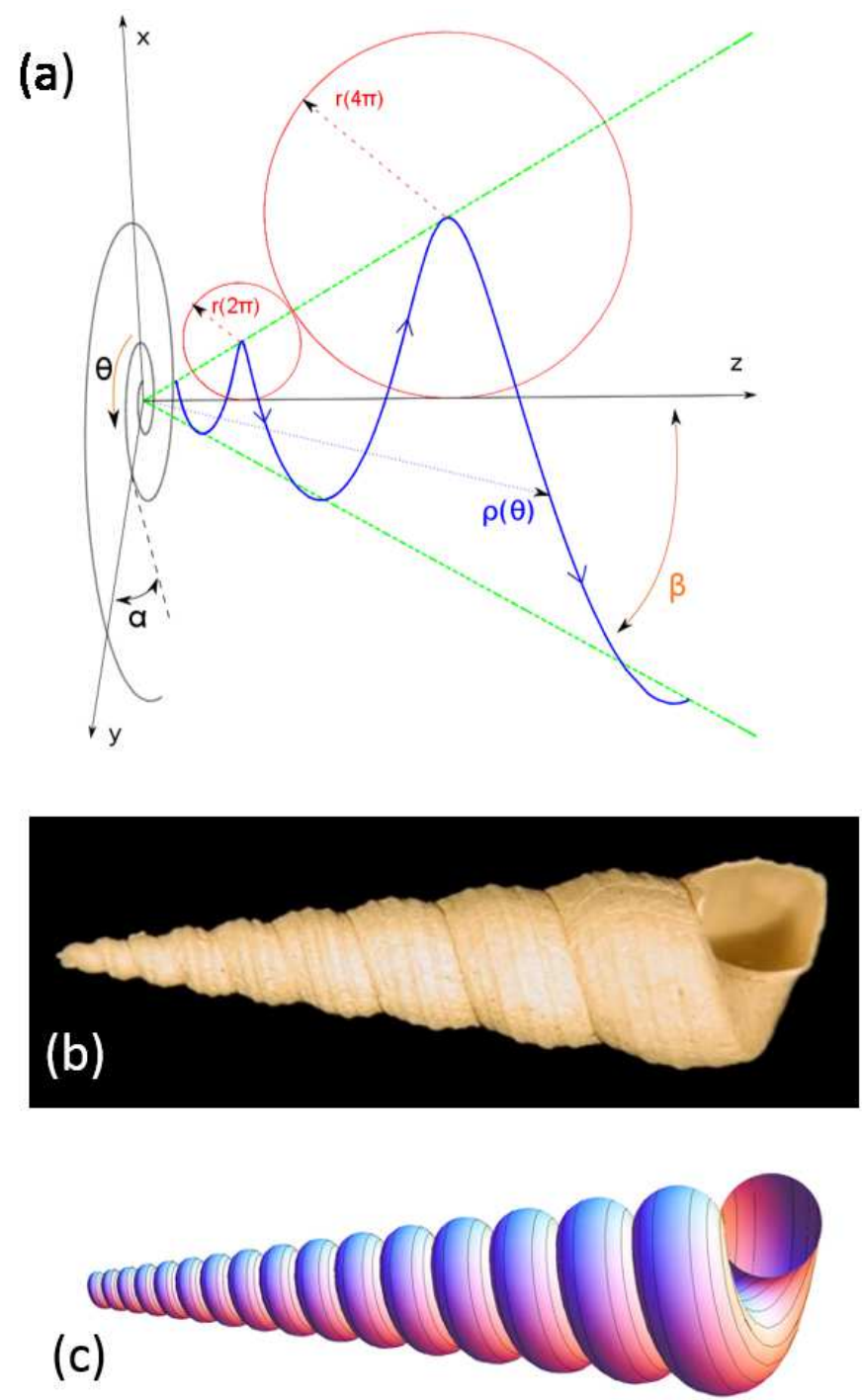

Fig. 4 (a) Parametric description of the growing seashell: the generating line is given by the mantle position $\rho(\theta)$ along a helico-spiral (blue line), while new material is deposited on a circular border with radius $r(\theta)$ (red circles).(b) Picture of the seashell turritella communis (b) and its simulated morphology (c). The simulation is performed using Eqs.(38- 41), setting the values $\mathbf{v}^{-}=0, \beta=$ $4^{o}, \alpha=\cot ^{-1}\left(\log \left(\frac{1+\sin \beta}{1-\sin \beta}\right) / 2 \pi\right)=87.72^{\circ}, m=1, \gamma=2 m \cot \alpha=0.044$.

The proposed model is applied to describe the shape of Turritella communis), a marine gastropod of the family of the Turritellidae, depicted in in Figure 4(b) . Its 
peculiarity resides by the fact that its coiling edge keeps tangent to the $\mathrm{z}$ axis, so that $\rho(\theta)=r(\theta / k) \sin \beta$.

This geometrical property, used in combination with Eq.(40), allows a correlation between the movement of the mantle and its mass supply, being:

$$
2 \pi \cot \alpha=\log \left(\frac{1+\sin \beta}{1-\sin \beta}\right) ; \quad \gamma_{\Sigma}=2 \mathrm{k} \cot \alpha
$$

The resulting simulated morphology is depicted in Figure 4(c), demonstrating that the neural system of the gastropod is somehow able to regulate mass production in function of the coiling angle.

\section{Free-boundary morphogenesis for fluid-like living matter}

In the previous section, we have introduced a general chemo-mechanical theory of morphogenesis, which requires the specification of the constitutive equations describing the biorheological properties of living matter, possibly spanning from viscous fluids to soft solids. Let us first discuss a simple free-boundary morphogenetic problem for a fluid-like biological system from [18]. In particular, we will consider the chemotactic expansion of an aggregate of living cells, which will be modelled as am incompressible newtonian fluid, such that:

$$
\sigma=-p \mathbf{I}+\eta \mathbf{d}
$$

where $\mathrm{p}$ is an hydrostatic pressure and $\eta$ is the fluid viscosity.

From Eq.(15), the motion is governed by the Navier-Stokes equations:

$$
\rho \frac{d \mathbf{v}}{d t}=\rho \mathbf{b}_{v}-\operatorname{grad} p+\eta \nabla^{2} \mathbf{v}
$$

where $\nabla^{2}$ is the spatial laplacian operator, and $\rho$ is the constant spatial density of the cell, which is almost equal to the one of water.

\subsection{Definition of the chemotactic model in a Hele Shaw cell}

We consider an aggregate of living cells occupying the domain $x \leq x_{b}$, where $x_{b}$ is the rectilinear border, of an Hele Shaw cell, i.e. confined between two parallel flat plates separated by an small gap of length $\ell$. The cells are bounded by an inviscid fluid which occupies the domain $x>x_{b}$, as sketched in Figure 5 (a). Let $z=0$ be the plane in between the plates and $\ell<<L$, where $L$ is the characteristic macroscopic size of the morphogenetic pattern that we aim to observe. Accordingly, the 
component of the velocity in $z$ is much smaller than the two others, which are also governed by the following scaling [54]:

$$
\frac{\partial^{2} v_{i}}{\partial x^{2}}<<\frac{\partial^{2} v_{i}}{\partial z^{2}}
$$

where the subscript $i=(x, y)$ indicates the component of the velocity. Since the typical duplication time of cells is about a day and the fluid is highly viscous, the corresponding flow occurs at a low Reynolds number, so that the terms $\eta \nabla^{2} \mathbf{v}$ dominate with respect to convective and gravitational effects, so that Eq. (43) simplifies in virtue of Eq.(44) as:

$$
\operatorname{grad} p=\eta \frac{\partial^{2} v_{i}}{\partial z^{2}}
$$

Respecting the necessary symmetry and boundary condition at the plates, we can therefore assume that:

$$
v_{i}(x, y, z ; t)=v_{i}(x, y, 0 ; t)\left(1-\frac{4 z^{2}}{\ell^{2}}\right) \quad i=(x, y)
$$

so that Eq.(45) reduces to a classical Darcy equation for the planar velocity $\mathbf{v}=$ $\mathbf{v}(x, y, 0, ; t)$, being:

$$
\mathbf{v}=\frac{\ell^{2}}{8 \eta} \operatorname{grad} p=K_{p} \operatorname{grad} p
$$

where all field and operators are planar. The parameter $K_{p}=\ell^{2} /(8 \eta)$ is the equivalent of the porosity coefficient, and represents here the friction forces resulting from the shallow geometry. It is therefore possible to solve the problem using a fully twodimensional model.

We assume that cell proliferation if driven by a morphogenetic signal diffusing inside the Hele-Shaw cell, and possessing an infinite reservoir at $x \rightarrow \infty$, which is independent on the local dynamics (Figure 5, b). The cells possess specific surface receptors which capture the morphogen at a typical uptake rate $\gamma_{c}$. Accordingly, the concentration $c=c(x, y ; t)$ of the morphogen is governed by the following reactiondiffusion equations inside the Hele-Shaw cell:

$$
\dot{c}=\left\{\begin{array}{lll}
D_{c} \nabla^{2} c-\gamma_{c} c & \text { if } & x \leq x_{b} \\
D_{c} \nabla^{2} c & \text { if } & x>x_{b}
\end{array}\right.
$$

where $D_{c}$ is the diffusion coefficient inside the cell.

The living cells respond to a gradient of the morphogen concentration by generating a mass flux $\mathbf{m}$. In particular, we assume the classical Keller-Siegel constitutive equation [71], being:

$$
\mathbf{m}=K_{c} \operatorname{grad} n
$$

where $K_{c}$ is a positive definite mobility parameter. Although possibly dependent on $c$ for representing a degenerate mobility in different classes of biochemical reactions, 

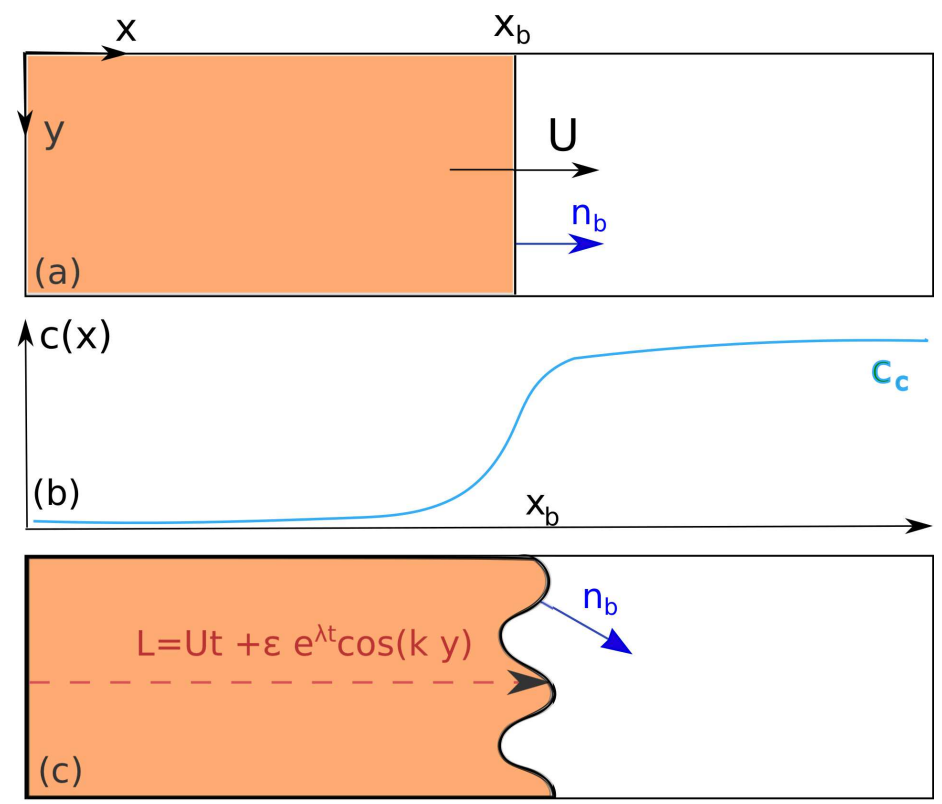

Fig. 5 Schematics of the Hele Shaw cell containing a living material (orange) expanding with a rectilinear free border at $x=x_{b}$ (a). Distribution of the morphogen concentration $n$ (green line) inside the cell (b). Perturbation of the rectilinear border with wavenumber $k$ and growth rate $\lambda$ (c).

for the sake of simplicity we assume $K_{c}$ to be a constant in the following. From Eq.(12), the balance of mass within the expanding cellular domain is given by:

$$
\frac{d \rho}{d t}=\operatorname{divm}-\rho \operatorname{div} \mathbf{v}=0
$$

which vanishes in virtue of the incompressibility of the living cells. In Eq.(50) we considered that the morphogenesis only resides on mass diffusion, neglecting the presence of volumetric sources of mass.

Substituting Eq.(47, refconst-m) in Eq.(50), the equation of motion reads:

$$
K_{p} \rho \nabla^{2} p-K_{c} \nabla^{2} c=0
$$

Thus, the Eqs. $(48,51)$ govern the morphogenesis of the free boundary domain in this model. The mathematical model is complemented by four boundary conditions at the free border. First, the Young-Laplace equation imposes the mechanical equilibrium at the interface, being:

$$
p=p_{0}-\sigma C \quad \text { at } \quad x=x_{b}
$$

where $C$ is the local curvature (initially equal to zero for a planar front), $\sigma$ is the surface tension and $p_{0}$ is the constant outer pressure of the inviscid fluid. Second, for 
compatibility we must impose the continuity of the normal velocity at the interface:

$$
\frac{d \mathbf{x}_{b}}{d t} \cdot \mathbf{n}_{b}=\mathbf{v}\left(x_{b}\right) \cdot \mathbf{n}_{b} \quad \text { at } \quad x=x_{b}
$$

where $\mathbf{n}_{b}$ is the outward unit vector at the free surface. Finally, two conditions must be imposed for $c$ and its normal gradient at the interface:

$$
c\left(x_{b}^{-}\right)=c\left(x_{b}^{+}\right) ; \quad \operatorname{grad} c\left(x_{b}^{-}\right) \cdot \mathbf{n}_{b}=\operatorname{grad} c\left(x_{b}^{+}\right) \cdot \mathbf{n}_{b}
$$

stating the continuity of the morphogen concentration and of the chemical flux across the free boundary, given the absence of any interfacial phenomenon.

\subsection{Dimensionless form of the governing equations}

The governing equation can be recast in a dimensionless form using the following values:

$$
\begin{array}{r}
t_{c}=\gamma_{n}^{-1} ; \quad L_{c}=\sqrt{\frac{D_{c}}{\gamma_{c}}} ; \quad v_{c}=\sqrt{D_{c} \gamma_{c}} ; \\
p_{c}=\frac{D_{c}}{K_{p}} ; \quad c_{c}=c(x \rightarrow \infty)
\end{array}
$$

where $t_{c}, L_{c}, v_{c}, p_{c}, n_{c}$ are characteristic time, length, velocity, pressure, and chemical concentration, respectively. Defining dimensionless variables with respect to such characteristic values to obtain, we can rewrite the Eqs. $(48,15,51)$ in their dimensionless form as follows:

$$
\begin{gathered}
\dot{\bar{c}}=\left\{\begin{array}{lll}
\bar{\nabla}^{2} \bar{c}-\bar{c} \text { if } & \bar{x} \leq \bar{x}_{b} \\
\bar{\nabla}^{2} \bar{c} & \text { if } & \bar{x}>\bar{x}_{b}
\end{array}\right. \\
\overline{\mathbf{v}}=-\operatorname{grad} \bar{p} \\
\bar{\nabla}^{2} \overline{\mathrm{p}}=-\bar{\nabla}^{2} \bar{n} ; \quad \text { with } \quad \mathrm{G}=\frac{\mathrm{K}_{\mathrm{c}} \mathrm{c}_{\mathrm{c}}}{\rho \mathrm{D}_{\mathrm{n}}}
\end{gathered}
$$

where the bar indicates dimensionless variables. Interestingly, the only resulting dimensionless parameter is $G$ in Eq.(58), which represents a ratio between morphogen-driven mass production and the mass evacuation rate driven by viscous effects. Furthermore, the boundary conditions given by Eqs.(52-54) rewrite as:

$$
\begin{gathered}
\bar{p}=\bar{p}_{0}-\bar{\sigma} \bar{C} ; \quad \text { with } \quad \bar{\sigma}=\frac{\sigma K_{p}}{D_{n}} \sqrt{\frac{\gamma_{n}}{D_{n}}=\frac{\sigma \ell^{2}}{8 \eta v_{c} L_{c}^{2}}} \text { at } \quad \bar{x}=\bar{x}_{b} \\
\frac{d \overline{\mathbf{x}}_{b}}{d \bar{t}} \cdot \mathbf{n}_{b}=\overline{\mathbf{v}}\left(x_{b}\right) \cdot \mathbf{n}_{b} \\
\bar{c}\left(\bar{x}_{b}^{-}\right)=\bar{c}\left(\bar{x}_{b}^{+}\right) ; \quad \bar{\nabla} \bar{c}\left(\bar{x}_{b}^{-}\right) \cdot \mathbf{c}_{b}=\bar{\nabla} \bar{c}\left(\bar{x}_{b}^{+}\right) \cdot \mathbf{n}_{b}
\end{gathered}
$$


In accordance with the Buckingham $\Pi$ theorem [13], the mathematical model given by Eqs.(56-61) is entirely governed by the dimensionless parameters $G$ and $\bar{\sigma}$ defined in Eqs. $(58,59)$, which will control the evolution of the biological system during morphogenesis. In physical terms, $\bar{\sigma}$ represents the ratio between the surface tension and the viscous forces.

For matters of notation compactness, we'll drop the bars in the following analysis.

\subsection{Traveling wave solution}

Eqs.(56-61) allow the existence of a traveling wave solution of the problem. Thus, we hypothesize that the front moves with a constant dimensionless velocity $\mathrm{U}$ going from left to right, i.e. assuming a positive chemotaxis driving towards the highest concentration of the morphogen. Considering a dimensionless variable $\zeta=(x-U t)$, we can solve the reaction-diffusion dynamics in Eqs. $(56,61)$, deriving the following morphogen concentration:

$$
c_{0}(\zeta)=\left\{\begin{array}{lll}
c_{0}(0) \cdot e^{\frac{-U+\sqrt{U^{2}+4}}{2}} & \text { if } & \zeta \leq 0 \\
1+\left(c_{0}(0)-1\right) \cdot e^{-U \zeta} & \text { if } & \zeta>0
\end{array}\right.
$$

where $c(-\infty)=0, c(\infty)=1$ and $c_{0}(0)=\frac{2 U}{U+\sqrt{U^{2}+4}}$ is the morphogen concentration at the rectilinear front. We remark that $c_{0}$ must be positive definite, so that the boundary expands with $U>0$. Imposing in Eqs. $(58,59)$ that $p_{0}(0)=p_{0}$ and that $p(-\infty)$ must be bounded, the traveling wave for the pressure field reads:

$$
p_{0}(\zeta)=-G\left(c_{0}(\zeta)-c_{0}(0)\right)+p_{0} \quad \text { if } \quad \zeta \leq 0
$$

Finally, the Dirichlet condition at the free boundary in Eq.(60) fixes the velocity of the traveling wave as:

$$
U=\frac{G-1}{\sqrt{G}}
$$

revealing that a traveling wave solution can appear if and only if $G>1$, i.e. if the chemotactic growth production is faster than diffusion-driven mass evacuation.

\subsection{Linear stability analysis}

Let us now study the stability of the traveling wave solution given by Eqs.(62,63), in order to investigate the existence of a morphological transition during the morphogenetic process. For this purpose, we impose a perturbation $\zeta(0)$ at the free boundary, whose dimensionless expression in the moving frame is given by : 


$$
\zeta(0)=x_{b}-U t=\varepsilon \cdot e^{\lambda t} \cos (\kappa y)
$$

where $\kappa$ is the spatial wavenumber and $\lambda$ is its time growth rate, as depicted in Figure 5(c).

We write $p$ and $c$ as a series expansion around the traveling wave solution in the moving frame, as follows:

$$
\begin{aligned}
& p(x, y ; t)=p_{0}(\zeta)+\varepsilon \cdot p_{1}(\zeta) e^{\lambda t} \cos (\kappa y) \\
& c(x, y ; t)=c_{0}(\zeta)+\varepsilon \cdot c_{1}(\zeta) e^{\lambda t} \cos (\kappa y)
\end{aligned}
$$

where $p_{0}(Z), c_{0}(Z)$ are the known traveling wave solutions.

The solution for $c_{1}(\zeta)$ can be found from Eq.(56), imposing the continuity of the chemical concentration and its flux across the boundary:

$$
c_{1}(\zeta)=\left\{\begin{array}{lll}
c_{1}(0) \cdot e^{\frac{-U+\sqrt{U^{2}+4\left(1+\lambda+\kappa^{2}\right)}}{2}} \zeta \text { if } & \zeta \leq 0 \\
c_{1}(0) \cdot e^{\frac{-U-\sqrt{U^{2}+4\left(\lambda+\kappa^{2}\right)}}{2}} \zeta & \text { if } \quad \zeta>0
\end{array}\right.
$$

where:

$$
c_{1}(0)=-\frac{4 U \cdot\left(U+\sqrt{U^{2}+4}\right)^{-1}}{\sqrt{U^{2}+4\left(1+\lambda+\kappa^{2}\right)}+\sqrt{U^{2}+4\left(\lambda+\kappa^{2}\right)}}
$$

Substituting the solution for $c_{1}(\zeta)$ in Eq.(58), we derive the leading order governing equation for the perturbed pressure field, being:

$$
\begin{aligned}
& p_{1}^{\prime \prime}(\zeta)-\kappa^{2} p_{1}(\zeta)+ \\
& +B n_{1}(0) \cdot\left[\left(\frac{-U+\sqrt{U^{2}+4\left(1+\lambda+\kappa^{2}\right)}}{2}\right)^{2}-\kappa^{2}\right] e^{\frac{-U+\sqrt{U^{2}+4\left(1+\lambda+\kappa^{2}\right)}}{2} \zeta}=0
\end{aligned}
$$

which is valid for $\zeta \leq 0$, and the prime denotes derivative on $\zeta$. Eq.(70) has the following general solution:

$$
p_{1}(\zeta)=-G c_{1}(0) e^{\frac{-U+\sqrt{U^{2}+4\left(1+\lambda+\kappa^{2}\right)}}{2}} \zeta A \cdot e^{\kappa \zeta}
$$

The constant $A$ in Eq.(71) can be fixed imposing the boundary solution in Eq.(59). Recalling that the curvature can be written as

$$
C=\frac{\partial^{2} \zeta(0) / \partial y^{2}}{\left[1+(\partial \zeta(0) / \partial y)^{2}\right]^{3 / 2}},
$$

we find that:

$$
p_{1}\left(\varepsilon \cdot e^{\lambda t} \cos (\kappa y)\right)=p_{0}(0)+\left(p_{0}{ }^{\prime}(0)+p_{1}(0)\right) \varepsilon \cdot e^{\lambda t}=p_{o}+\bar{\sigma} \kappa^{2} \varepsilon \cdot e^{\lambda t} \cos (\kappa y)
$$


giving at the leading order:

$$
A=\bar{\sigma} \kappa^{2}+G\left[c_{1}(0)+c_{0}^{\prime}(0)\right]=\bar{\sigma} \kappa^{2}+U+G n_{1}(0)
$$

Finally, the boundary condition in Eq.(60) allows deriving the dispersion equation for the perturbed mode. Accordingly, we find that:

$$
-p_{0}^{\prime \prime}(0)-p_{1}^{\prime}(0)=\lambda
$$

which can be simplified as:

$$
\lambda=-\bar{\sigma} \kappa^{3}-\kappa U+G\left[n_{0}^{\prime \prime}(0)+c_{1}(0)\left(-\kappa+\frac{-U+\sqrt{U^{2}+4\left(1+\lambda+\kappa^{2}\right)}}{2}\right)\right]
$$

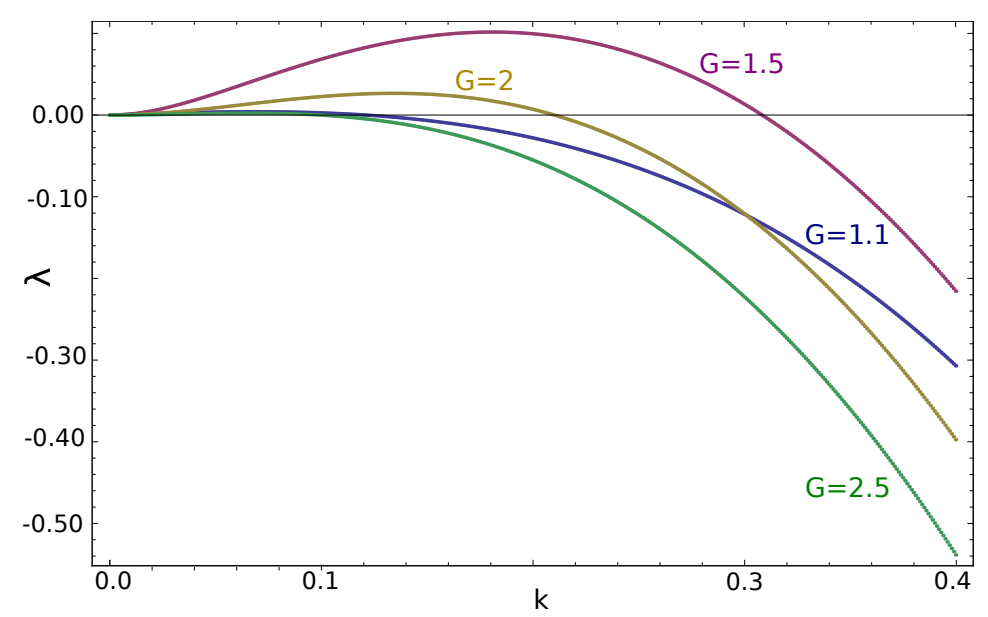

Fig. 6 Dispersion diagrams shown at different values of the dimensionless parameter $G=\frac{K_{m} K_{\Psi} n_{c}}{\rho D_{n}}$. The curves have been numerically obtained using the Newton algorithm, setting $\sigma=0.5$.

Substituting Eqs.(64, 69) in Eq.(76), we can can write an implicit dispersion equation uniquely as a function of the two dimensionless parameters $G, \bar{\sigma}$ of the problem, reading:

$\lambda=-\bar{\sigma} \kappa^{3}-\kappa \frac{G-1}{\sqrt{G}}+\frac{G-1}{G}-\frac{(G-1) \cdot\left(-2 \kappa-\frac{G-1}{\sqrt{G}}+\sqrt{\frac{(G+1)^{2}}{G}+4\left(\lambda+\kappa^{2}\right)}\right)}{\sqrt{\frac{(G-1)^{2}}{G}+4\left(\lambda+\kappa^{2}\right)}+\sqrt{\frac{(G+1)^{2}}{G}+4\left(\lambda+\kappa^{2}\right)}}$

Since Eq.(77) involves the dimensionless growth mode $\lambda$ and wavenumber $\kappa$ in an implicit way, we solved it numerically. The resulting curves are depicted in Figures ?? and 7 for different values of the dimensionless parameters $G$ and $\bar{\sigma}$. Even if both 


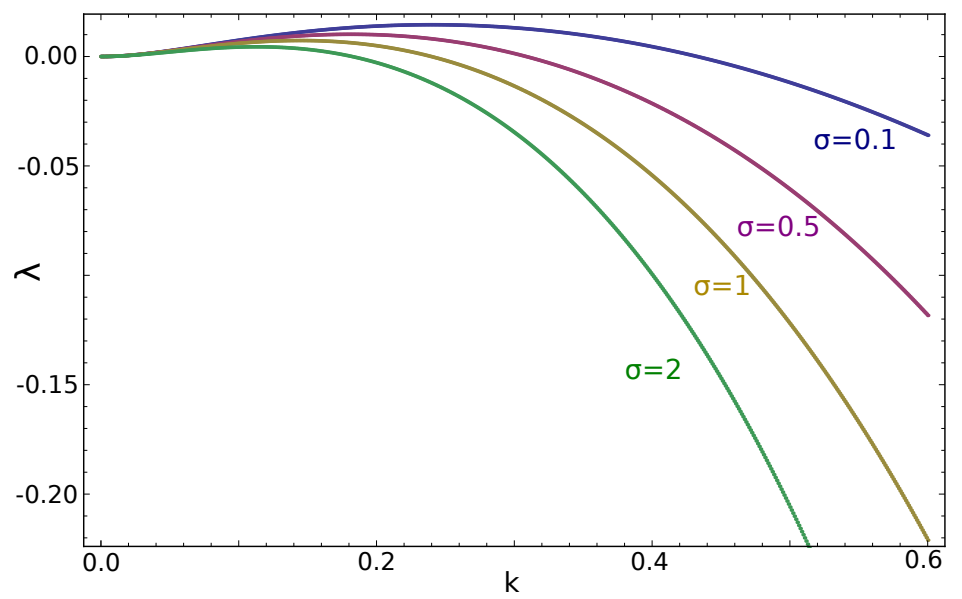

Fig. 7 Dispersion diagrams shown at different values of the dimensionless parameter $\bar{\sigma}=$ $\frac{\sigma K_{p}}{D_{n}} \sqrt{\frac{\gamma_{n}}{D_{n}}}$. The curves have been numerically obtained using the Newton algorithm, setting $G=1.5$.

the surface tension and the boundary velocity are stabilizing effects at small wavelengths, the rectilinear front is always unstable at large wavelengths, having a maximum value $\lambda_{\max }$ of the time growth rate. Although the a nonlinear stability analysis would be required for investigating the pattern dynamics, we expect the emergence of undulated structures with a typical length of about $2 \pi / \kappa\left(\lambda_{\max }\right) \cdot \sqrt{D_{n} / \gamma_{n}}$.

\subsection{Pattern formation in the nonlinear regime}

The dispersion diagrams resulting from the linear stability analysis highlighted that the rectilinear border is always unstable at large wavelengths. The dispersion curves are qualitatively similar to the one encountered in other hydrodynamic problems, such as the Saffmann-Taylor [101] or the Mullins-Sekerka [89] instabilities. Similarly, we expect that the moving front will rapidly develop growing undulations, whose shape will be fixed by fully nonlinear effects. In the following we numerically investigate the nonlinear pattern formation for the proposed model in order to describe some experimental results on epithelial cells [94], showing the formation of fingering instabilities in expanding free rectilinear surfaces of cellular monolayers. In order to provide a computational solution, we implemented the problem into a finite element code [49]. Eqs. $(56,58)$ have been solved on a triangular grid, fitting at every iteration the moving interface. Briefly, given the concentration of nutrients at time $t_{m}$, the pressure $p_{m}$ is first computed, through Eq. (58) and then the velocity field, using the Darcys law. Thus, the boundary can be explicitly moved and Eq. (56) can be solved for the concentration at time $t_{m+1}$, using an implicit-Euler scheme. In order to guarantee the robustness of the semi-implicit algorithm, at every iteration, 

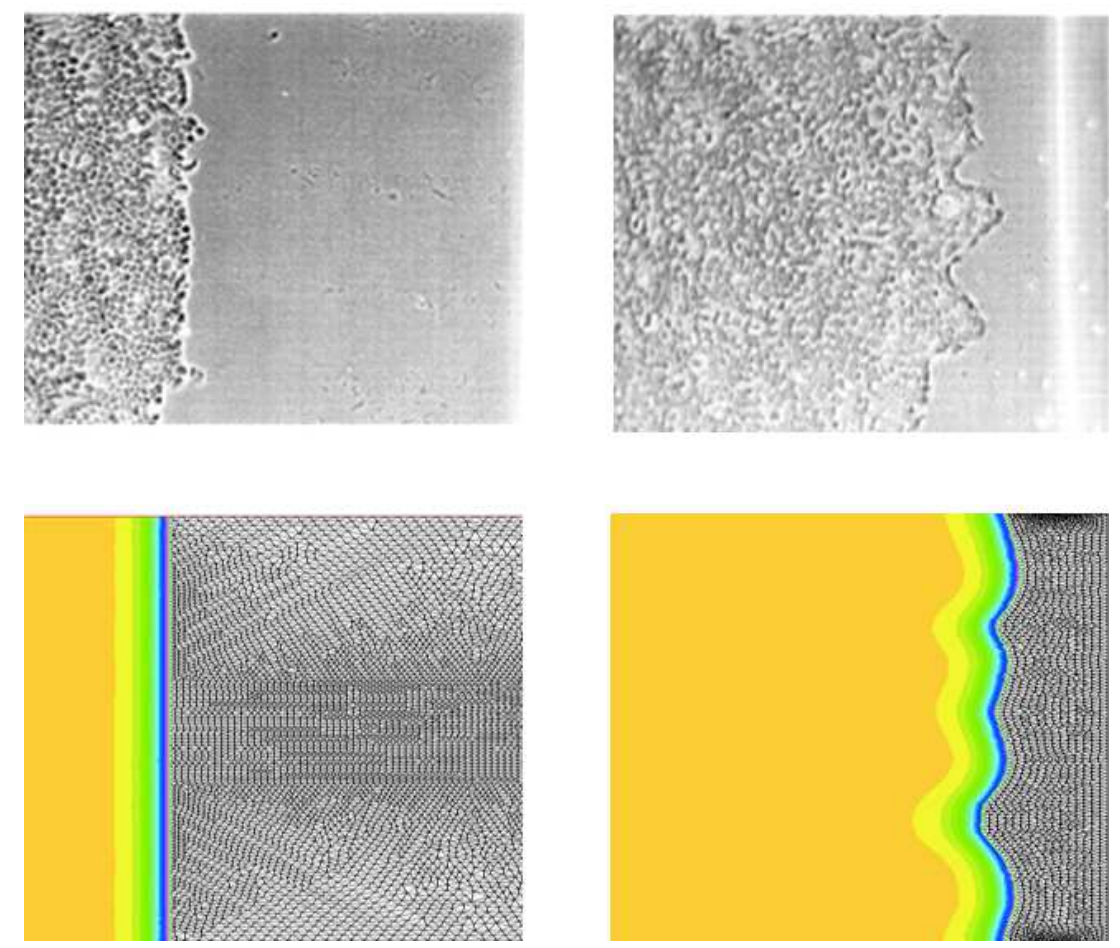

Fig. 8 (a) Wound healing experiment showing the propagation over $24 h$ of a monolayer of tumour cells. Adapted from [108]. (b) Fully nonlinear numerical simulation of the proposed free-boundary morphogenetic model.

the time step $\Delta t=\left(t_{m+1}-t_{m}\right)$ is set equal to the ratio between the minimum mesh size and the modulus of the maximum boundary velocity at the $m^{\text {th }}$ iteration. Finally, a spatial discretization with finite element is performed using continuous piecewise quadratic $P 2$ Lagrangian elements. Since the mesh size affects the minimum observable unstable wavelength, we adaptively refined the mesh in order to control the number of elements on the colony contour at every time step.

Simulation results are shown in Figure 8 against some pictures of a wound healing experiment showing the propagation over $24 h$ of a monolayer of tumour cells. The qualitative agreement of the emerging morphology highlights the importance of chemo-mechanical cues on the dynamics of the wound healing processes, whose effectiveness is known depend on the coupling between the motility and the cell proliferation rate [82]. 


\section{Growth, remodelling and morphogenesis for soft elastic matter}

The proposed morphogenetic theory should be refined to account for further important issues arising when dealing with solid living matter. Firstly, biological tissues undergo large deformations. Therefore, the undeformed and deformed configurations are not necessarily close, so that the classical infinitesimal elastic theory cannot be used. Secondly, soft biological matter exhibit residual stresses, i.e. a selfequilibrated distribution of internal stresses persists in the tissue when all external loads have been removed. Indeed, as discussed in the previous sections, living materials have the striking ability to change actively their micro-structure for adjusting to the surrounding media, developing a state of internal tension aimed at optimizing their structural behavior. Residual stresses in living materials, closely resembling the ones originated by misfits within inert matter [3], are almost ubiquitous in Nature). Their magnitude has been measured in several tissues (e.g. arteries [17], airways [57], hearth [124], gastro-intestinal tissues [53]), using simple cutting experiments. In arteries, for instance, they can be inferred by measuring the opening angle of an excised tissue ring after a radial cut. Thirdly, residual stress may accumulate in the tissue during a morphogentic process, possibly triggering an elastic instability beyond a critical value. Accordingly, they can orchestrate a morphological transition even in the absence of either external loads or geometrical constraints. Lastly, soft tissues exhibit a nonlinear elastic behavior which should be taken into account when making constitutive assumptions.

In the following, we will extend the proposed chemo-mechanical theory of open systems in view of these key considerations.

\subsection{An interpretation of morphogenesis in solids using the theory of configurational forces}

The theory of configurational forces allows to provide a straightforward interpretation of the driving forces acting during the morphogenetic processes in living matter [85]. For a soft elastic solid, we can postulate the existence of an Helmholtz free energy $\Psi$ having the following functional form:

$$
\Psi=\Psi\left(\mathbf{F}, c_{i}, \Theta ; \mathbf{X}, t\right)
$$

where the explicit dependence on $\mathbf{X}, t$ may represent growth and ageing phenomena, respectively. Applying the chain rule for the differentiation of $\Psi$ in in Eq.(28), we obtain the following set of constitutive equations [30] :

$$
\mathbf{S}=\rho_{0} \frac{\partial \Psi}{\partial \mathbf{F}} ; \quad \Psi_{i}=\rho_{0} \frac{\partial \Psi}{\partial c_{i}} ; \quad \eta=-\frac{\partial \Psi}{\partial \Theta}
$$

Accordingly, the reduced Clausius-Duhem inequality rewrites: 


$$
\mathbf{J}_{c i} \cdot\left(\operatorname{Grad} \Psi_{i}+\eta_{i} \operatorname{Grad} \Theta\right)-\left.\rho_{0} \frac{\partial \Psi}{\partial t}\right|_{\text {expl }}-\frac{\mathbf{Q}}{\Theta} \cdot \operatorname{Grad} \Theta \geq 0
$$

where the explicit derivative over time accounts for possible dissipation during ageing phenomena [83].

The theory of configurational forces proves that canonical projection of the balance of the linear momentum in the material setting allows accounting for the momentum associated to all the degrees of freedom of the deformation field, while the balance of $\mathbf{p}$ involves only the translational momentum [84]. This is of fundamental importance for morphogenetic processes, which are characterized by the presence of inhomogeneities in the material set. Let $\mathbf{V}=\left.\frac{\partial \mathbf{X}}{\partial t}\right|_{x}=-\mathbf{F}^{-1} \mathbf{v}$ be the inverse motion velocity, i.e. the material velocity field, we can define a pseudomomentum density or canonical momentum density as follows:

$$
\mathbf{P}_{m}=\frac{\partial K}{\partial \mathbf{V}}=\rho_{0} \mathbf{F}^{T} \mathbf{F V}=-\mathbf{F}^{T} \mathbf{p}=-\mathbf{p F}
$$

where $K=1 / 2 \rho_{0} \mathbf{v}^{2}$ is the kinetic energy. Let us now perform a right-multiplication of Eq.(17) times $\mathbf{F}$, as follows:

$$
\frac{d}{d t}\left(\rho_{0} \mathbf{v}\right) \mathbf{F}=-\frac{d \mathbf{P}_{m}}{d t}-\rho_{0} \mathbf{v} \dot{\mathbf{F}}=\mathbf{b}_{v} \mathbf{F}-\frac{\Omega}{\rho_{0}} \mathbf{P}_{m}+(\operatorname{Div} \mathbf{S}) \mathbf{F}
$$

Using the constitutive assumptions in Eq.(142), let us recall the useful identities:

$$
\begin{gathered}
(\operatorname{Div} . \mathbf{S}) \cdot \mathbf{F}=\operatorname{Div} .(\mathbf{S F})-\operatorname{tr}(\mathbf{S} \operatorname{Grad} \mathbf{F}) \\
\operatorname{tr}(\mathbf{S} \operatorname{Grad} \mathbf{F})=\operatorname{Div} .\left(\rho_{0} \Psi \mathbf{I}\right)-\left.\frac{\partial\left(\rho_{0} \Psi\right)}{\partial \mathbf{X}}\right|_{\text {expl }}-\mu_{i} \operatorname{Grad} c_{i}+\eta \operatorname{Grad} \Theta \\
\rho_{0} \mathbf{v} \dot{\mathbf{F}}=\operatorname{Grad} K-\frac{1}{2} \mathbf{v}^{2}\left(\operatorname{Grad} \rho_{0}\right)
\end{gathered}
$$

Substitute Eqs.(13 83-85) in Eq.(82), and we find the the canonical balance of pseudomomentum density in the material framework:

$$
\frac{d \mathbf{P}_{m}}{d t}=\mathbf{f}^{e x t}+\mathbf{f}^{g}+\mathbf{f}^{i n h}+\mathbf{f}^{c}+\mathbf{f}^{\Theta}+\operatorname{Div} \mathbf{b}
$$

Eq.(86) states that there are five sources of material inhomogeneities: the convection of the body forces in $\mathbf{f}^{\text {ext }}$, the mas growth in $\mathbf{f}^{g}$, the true material inhomogeneities in $\mathbf{f}^{\text {inh }}$, the internal variables in $\mathbf{f}^{c}$, and the temperature in $\mathbf{f}^{\Theta}$. They are defined as:

$$
\left\{\begin{array}{l}
\mathbf{f}^{e x t}=-\mathbf{b}_{v} \mathbf{F} \\
\mathbf{f}^{g}=\frac{\rho_{0}}{\rho_{0}} \mathbf{P}_{m} \\
\mathbf{f}^{i n h}=\left.\frac{\partial\left(K-\rho_{0} \Psi\right)}{\partial \mathbf{X}}\right|_{\operatorname{expl}} \\
\mathbf{f}^{c}=-\mu_{i} \mathrm{Grad} c_{i} \\
\mathbf{f}^{\Theta}=\rho_{0} \eta \operatorname{Grad} \Theta
\end{array}\right.
$$


Moreover, we find the stress measure that drives the evolution of material inhomogeneities is the Eshelby tensor $\mathbf{b}$, defined as:

$$
\mathbf{b}=-\left[\left(K-\rho_{0} \Psi\right) \mathbf{I}+\mathbf{S F}\right]
$$

This stress measure is a fundamental importance since it governs the configurational changes during morphogenesis, as it will be highlighted in the following . Finally, we recall that the frame indifference of the Helmholtz free energy in Eqs.(79) with respect of arbitrary rotations of the actual configuration allows deriving the conservation of the angular momentum.

\subsection{Mathematical theory of volumetric growth in soft solids}

At the beginning of the 1980s, Skalak and coworkers proposed the firs kinematic descriptions of finite volumetric and surface growth $[104,103]$ in a continuum mechanics framework. They introduced the idea that growth can induce geometric incompatibilities in the microstructure, which would provoke the transition from an unloaded stress-free configuration to a pre-stressed reference configuration . Conversely, no internal stresses arise in the tissue if the growth strains are compatible, i.e. do not induce material distorsions.

The seminal work of Skalak opened the door to a number of experimental studies which aimed at characterizing the residual stress distribution in biological tissues. From a modeling viewpoint, much work has been done by Anne Hoger and coworkers $[62,63,64,65,70]$ to define a hyperelastic constitutive theory of soft materials with residual stresses. In particular, a multiplicative decomposition has been proposed for dealing with volumetric growth in living materials $[98,105]$. The mechanical approach based on a multiplicative decomposition was first introduced in the theory of elasto-plasticity by Kröner [74] and Lee [79] in order to split the inelastic and the elastic components and it has been widely employed in continuum mechanics models.

According to this mathematical theory, the mapping $\chi$ introduced in Eq.(1) can be split into two components. The first is associated to the a change of the unstressed state of the material and it transforms the tissue from its initial stress free configuration $\mathscr{B}_{0}$ into a new stress-free grown (remodeled) state, denoted as $\mathscr{B}_{g}$ in Figure 9. In mathematical terms, the deformation gradient $\mathbf{F}$ defined in Eq.(2) can be split into two components, as follows:

$$
\mathbf{F}=\mathbf{F}_{e} \mathbf{F}_{g}
$$

where $\mathbf{F}_{g}$ represents the volumetric growth (remodeling) tensor and $\mathbf{F}_{e}$ is the elastic tensor. We remark that the intermediate state is not necessarily a configuration that could be physically observable for the material. Indeed, this grown or remodeled state $\mathscr{B}_{g}$ can be seen as the collection of local states obtained at each material point 


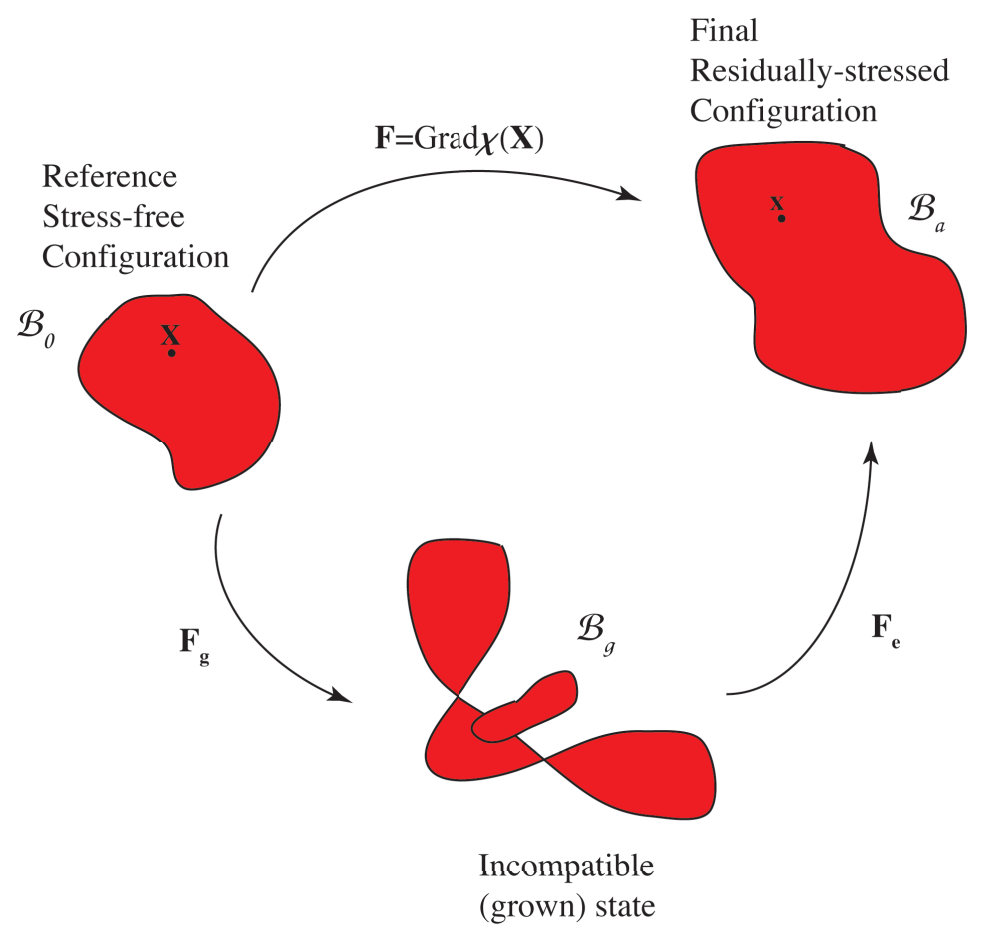

Fig. 9 The multiplicative decomposition of the deformation gradient $\mathbf{F}$ : the growth (remodeling) component $\mathbf{F}_{g}$ defines a natural grown or remodeled state $\mathscr{B}_{g}$, in which geometrical incompatibilities are allowed, and the elastic component $\mathbf{F}_{e}$, which restores the physical compatibility in the spatial configuration.

if all the surrounding constraints would be removed. The ensemble of these stressfree parts defines the natural grown state of the tissue $\mathscr{B}_{g}$. Since they may not be geometrically compatible with each other, overlapping and intersections are allowed in $\mathscr{B}_{g}$. The elastic component later restores the global compatibility $\mathrm{n}$ the spatial configuration $\mathscr{B}_{a}$ from the intermediate state $\mathscr{B}_{g}$.

It is worth noting that even if $\mathbf{F}$ is the geometrical gradient of a deformation, this may not be the case for its components $\mathbf{F}_{g}$ and $\mathbf{F}_{e}$, which are defined as tangent maps as follows:

$$
\mathbf{F}_{g}: T \mathscr{B}_{0} \rightarrow T \mathscr{B}_{g} \quad \mathbf{F}_{e}: T \mathscr{B}_{g} \rightarrow T \mathscr{B}_{a}
$$

The multiplicative decomposition in Eq.(122) implies that the morphogenetic effects on the tissue strain can be considered separately from the elastic deformations. This assumption resides on the physical observation that the typical time-scales involving elasticity and growth (remodeling) are very different.

Let $\tau_{g}, \tau_{v}, \tau_{l}, \tau_{e}$ be the four characteristic times of the growth elastic problem: 
- The characteristic time of growth $\tau_{g}$ can range from hours to days, e.g. being characterized by the typical doubling time of living cells.

- The viscoelastic characteristic time $\tau_{v}$ is of the order of hundreds of seconds and is considered as the relaxation time of the tissue [46].

- The elastic time $\tau_{e}$ is associated to the characteristic propagation time of elastic waves, in the order of seconds.

- The loading time $\tau_{l}$ of the external loading

It is therefore apparent that the growth time scale is much bigger than the others, so that the growth strains can be separated from the elastic deformations. Furthermore, since the elastic response is much faster than the growth processes, the soft matter is always in a state of elastic equilibrium at times comparable to $\tau_{g}$ [50].

Under these assumptions, it is possible to introduce a material isomorphism for the Helmholtz free energy [40], so that:

$$
\rho_{0} \Psi\left(\mathbf{F}, c_{i}, \Theta ; \mathbf{X}, t\right)=\rho_{0} \Psi\left(\mathbf{F}, \mathbf{F}_{g}(\mathbf{X} ; \mathbf{t}), c_{\beta}, \Theta, t\right)=\rho_{g}\left(\operatorname{det} \mathbf{F}_{g}\right) \Psi_{0}\left(\mathbf{F}_{e}, c_{\beta}, \Theta, t\right)
$$

where $\rho_{g}=\left(\rho_{0} / \operatorname{det} \mathbf{F}_{g}\right)$ represents the mass density with respect the intermediate state. In practice, the mechanical behavior of the materials uniquely depends on the elastic part oh the deformation gradient, whilst the growth tensor may introduce volume changes and geometrical distortions.

Before considering the constitutive equation and the evolution laws for the growth tensor in the next paragraph, let us finally clarify the morphogenetic information carried within $\mathbf{F}_{g}$. Since most living materials are mainly composed by water, the incompressibility constraint reads:

$$
\operatorname{det} \mathbf{F}_{e}-1=0
$$

so that in general $\rho=\rho_{g}$. Accordingly $\left(\operatorname{det} \mathbf{F}_{g}\right)$ represent the volume ratio between the grown and the ungrown matter. If we assume that the material is produced at a constant density $\rho$, the mass balance Eq.(13) rewrites:

$$
\frac{\dot{\rho}_{0}}{\rho_{0}}=\frac{\Omega}{\rho_{0}}=\frac{\left(\operatorname{det} \mathbf{F}_{g}\right)}{\operatorname{det} \mathbf{F}_{g}}=\operatorname{tr}\left(\dot{\mathbf{F}}_{g} \mathbf{F}_{g}^{-1}\right)
$$

Eq.(93) highlights a fundamental issue: for an incompressible material, the specification of the functional dependence of $\mathbf{F}_{g}$ over time is equivalent to a constitutive assumption on $\Omega$. Therefore, only one of the two can be constitutively imposed. Whilst modeling fluid-like materials it was mandatory to define $\Omega$, in solid materials this could be equivalently done by imposing an evolution law for $\mathbf{F}_{g}$. 


\subsection{Constitutive assumptions and evolution laws for growth and remodelling}

Let us now discuss the thermodynamic restrictions arising from the application of the multiplicative decomposition in Eq.(122) with the material isomorphism in Eq.(91). Substituting Eq.(91) in the reduced dissipation inequality in Eq.(28), developing the differentions using the chain rule one obtains after straightforward calculation the following constitutive laws:

$$
\begin{gathered}
\sigma=\rho \mathbf{F}_{e} \cdot \frac{\partial \Psi_{0}}{\partial \mathbf{F}_{e}} \\
\mathbf{S}=\rho_{0} \mathbf{F}_{g}^{-1} \cdot \frac{\partial \Psi_{0}}{\partial \mathbf{F}_{e}}
\end{gathered}
$$

Using Eqs. $(95,93)$, the residual dissipation inequality inside the control volume reads:

$$
-\operatorname{tr}\left[\mathbf{b}\left(\mathbf{F}_{g}^{-1} \cdot \dot{\mathbf{F}}_{g}\right)\right]+\dot{\rho}_{0} \Psi_{0}-\rho_{0} \frac{\partial \Psi_{0}}{\partial t} \geq 0
$$

Eq.(96) highlights that the static material Eshelby stress tensor $\mathbf{b}=\rho_{0} \Psi_{0} \mathbf{I}-\mathbf{S F}$ is the driving stress measure directing the evolution dynamics of the morphogenetic processes. The frame indifference for the material isomorphism in Eq.(91) imposes that $b$ is a symmetric tensor. Accordingly, thermodynamically consistent evolution laws for growth and remodelling read:

$$
\dot{\mathbf{F}}_{g}=-f^{+}\left(c_{i}, \Theta\right) \rho_{0} \mathbf{F}_{g} \mathbf{b}
$$

where $f^{+}\left(c_{i}, \Theta\right)$ is a positive definite scalar function, whose expression may represents a specific temperature-dependent (e.g. Arrhenius-based relations) chemical kinetics in the reactions occurring during the morphogenetic processes [22]. The evolution law in Eq.(97) is a generalization of the classical results obtained for inelastic continua, since the Eshelby tensor is the symmetric stress conjugate of the growth velocity gradient [86]. Moreover, the symmetry of the driving force expressed by $\mathbf{b}$ is a necessary requirement, as pointed out by [103]: indeed, the symmetric part of $\mathbf{F}_{g}$ is the only shape-changing deformation of the growth tensor.

Let us now provide a geometrical interpretation of the coupling between stress and growth in the proposed mathematical model. For this purpose, we perform the explicit gradient of the free energy expressed by Eq.(91), and we derive the quasi-static driving force $\mathbf{f}_{i n h}$ of true material inhomogeneities in Eq.(87):

$$
\mathbf{f}_{i n h}=-\left.\rho_{0} \frac{\partial \Psi_{0}}{\partial \mathbf{X}}\right|_{\text {expl }}=-\rho_{0}\left(\frac{\partial \Psi}{\partial \mathbf{F}_{g}}: \operatorname{Grad} \mathbf{F}_{g}\right)=-\rho_{0} \mathbf{b}: \mathscr{T}
$$

where we used the two dots to indicate double contraction, i.e. $(\mathbf{b}: \mathscr{T})_{k}=\mathbf{b}_{j i}(\mathscr{T})_{i j k}$. In Eq.(98), the third-order tensor $\mathscr{T}=\mathbf{F}_{g}^{-1} \mathrm{Grad}_{\mathbf{F}_{g}}$ is the generally not-symmetric torsional material connection associated with $\mathbf{F}_{g}$. Accordingly, the expression of the 
driving force in Eq.(98) reflects the geometrical interpretation that the Eshelby force is the stress measure driving the torsion of the material connection during morphogenesis. Evolution laws coupling stress, growth and mass transport, extended to the second gradient of the deformation have been discussed in further details in [22], showing that the strain gradient Eshelby stress is associated to the curvature of the material connection.

\subsection{Morpho-elasticity of growing living matter}

Morpho-elasticity is the recently developed branch of continuum mechanics investigating the emergence of complex patterns in living matter after the occurrence of an elastic instability. Indeed, the accumulation of residual stresses during morphogenetic processes can trigger a morphological transition within the biological material as a result of an elastic bifurcation. In the following, we will present the basic theoretical tools in the fields.

\subsubsection{Basic solution of the quasi-static elastic problem}

In preceding parts of this section, we have shown that the thermo-mechanics of open systems and the theory of configurational forces are useful frameworks to model the chemo-mechanical cues of morphogenesis in soft elastic tissues.

Summarizing, the resulting boundary value problem in nonlinear elasticity is given by the following set of equations in the current configuration $\mathscr{B}_{a}$ :

$$
\left\{\begin{array}{l}
\rho \dot{\mathbf{v}}-\operatorname{div} \sigma-\mathbf{b}_{v}=0 \\
\omega=\rho \operatorname{tr}\left(\dot{\mathbf{F}}_{g} \mathbf{F}_{g}^{-1}\right) \\
\operatorname{det} \mathbf{F}_{e}=1 \\
\sigma=\rho \mathbf{F}_{e} \frac{\partial \psi_{0}}{\partial \mathbf{F}_{e}}-p \mathbf{I} \\
\dot{\mathbf{F}}_{g}=-f^{+}\left(c_{i}, \Theta\right) \rho_{0} \mathbf{F}_{g} \mathbf{b}
\end{array}\right.
$$

which contains the balances of linear momentum and mass, the incompressibility constraint, the constitutive equation for the Cauchy stress tensor and the evolution law for the growth tensor, respectively. Such governing equations are typically complemented by three possible sets of boundary conditions, which will be briefly discussed.

Let $\partial \mathscr{B}_{0}, \partial \mathscr{B}_{0}^{\mathbf{x}}, \partial \mathscr{B}_{0}^{\sigma}$ be the boundary in the reference configuration and two portions of the boundary, respectively, such that $\partial \mathscr{B}_{0}=\partial \mathscr{B}_{0}^{\mathbf{x}} \cup \mathscr{B}_{0}^{\sigma}$. Let $\partial \mathscr{B}_{a}, \partial \mathscr{B}_{a}^{\mathbf{x}}, \partial \mathscr{B}_{a}^{\sigma}$ the associated quantities in the actual configuration, where $\partial \mathscr{B}_{a}=\partial \mathscr{B}_{a}^{\mathbf{x}} \cup \mathscr{B}_{a}^{\sigma}$. Let us consider a quasi-static growth process, so that we are not interested in determining the evolution of the growth tensor $\mathbf{F}_{g}$ over time, but we aim at deriving the 
elastic solution at a fixed value of $\mathbf{F}_{g}$. Under this assumption, the three possible sets of boundary conditions read:

- Dirichlet boundary conditions: the displacements for all points belonging to $\partial \mathscr{B}_{0}$ and $\partial \mathscr{B}_{a}$ are specified in the following forms, respectively:

$$
\begin{cases}\mathbf{u}_{0}(\mathbf{X})=\mathbf{x}(\mathbf{X})-\mathbf{X}=\mathbf{u}_{0}^{*} & \text { on } \partial \mathscr{B}_{0} \\ \mathbf{u}_{a}(\mathbf{x})=\mathbf{x}-\mathbf{X}(\mathbf{x})=\mathbf{u}_{0}^{*} & \text { on } \partial \mathscr{B}_{a}\end{cases}
$$

where $\mathbf{u}_{0}, \mathbf{u}_{a}$ are the displacements in material and spacial form, respectively and $\mathbf{u}_{0}^{*}$ is the displacement vector to be assigned at the boundaries.

- Neumann boundary conditions: the traction loads at all points belonging to $\partial \mathscr{B}_{0}$ and $\partial \mathscr{B}_{a}$ are given in the following forms, respectively:

$$
\begin{cases}\mathbf{S}^{T} \mathbf{N}=\mathbf{t}_{0}^{*} & \text { on } \partial \mathscr{B}_{0} \\ \sigma \mathbf{n}=\mathbf{t}^{*} & \text { on } \partial \mathscr{B}_{a},\end{cases}
$$

where $\mathbf{S}, \sigma$ are the Nominal and Cauchy stress respectively and $\mathbf{t}_{0}^{*}, \mathbf{t}^{*}$ are traction vectors to be assigned.

- Mixed boundary conditions: the displacements are specified on a portion of the boundary $\partial \mathscr{B}_{0}^{\mathbf{x}}, \partial \mathscr{B}_{a}^{\mathbf{x}}$ and traction loads are given on the remaining subset $\partial \mathscr{B}_{0}^{\sigma}, \partial \mathscr{B}_{a}^{\sigma}$, in the following forms:

$$
\left\{\begin{array}{lr}
\mathbf{u}_{0}(\mathbf{X})=\mathbf{u}_{0}^{*} & \text { on } \partial \mathscr{B}_{0}^{\mathbf{x}} \\
\mathbf{S}^{T} \mathbf{N}=\mathbf{t}_{0}^{*} & \text { on } \partial \mathscr{B}_{0}^{\sigma}
\end{array}\right.
$$

in the reference configuration or:

$$
\begin{cases}\mathbf{u}_{a}(\mathbf{x})=\mathbf{u}_{0}^{*} & \text { on } \partial \mathscr{B}_{a}^{\mathbf{x}} \\ \sigma \mathbf{n}=\mathbf{t}^{*} & \text { on } \partial \mathscr{B}_{a}^{\sigma},\end{cases}
$$

in the actual configuration.

In quasi-static conditions, let $\mathbf{x}^{(0)}=\chi^{(0)}(\mathbf{X})$ be the basic solution of the governing equation in Eq.(99) wit one of the possible sets of boundary conditions in Eqs.(100-103) The solution $\mathbf{x}^{(0)}$ depends on a control parameter which is related to the given expression of $\mathbf{F}_{g}$. In the following, we will introduce the theory of incremental deformations superposed on finite strains to investigate the linear stability analysis of this basic solution.

\subsubsection{Method of incremental deformations superposed on finite deformations}

Following Ogden [91], the fundamental idea of the method of incremental deformations superposed on finite deformations is to perturb the basic solution $\mathbf{x}^{(0)}$ to the elastic problem, with a small incremental deformation. Performing a series expansion, the zeroth order term is a finite deformation, representing the basic solution with the initial shape of the material, whilst the first order term is an incremental 
deformation, defining the possible morphology after an elastic bifurcation.

Kinematics of the incremental deformation Let $\mathbf{x}^{(0)}$ be the basic position vector in $\mathscr{B}_{a}$ identified by the basic finite deformation $\chi^{(0)}$ and let $\varepsilon \hat{\mathbf{x}}$ be a small displacement from $\mathbf{x}^{(0)}$. Accordingly, the perturbed position $\overline{\mathbf{x}}$ can be written as:

$$
\overline{\mathbf{x}}=\mathbf{x}^{(0)}+\varepsilon \hat{\mathbf{x}}=\chi^{(0)}(\mathbf{X})+\varepsilon \chi^{(1)}\left(\mathbf{x}^{(0)}\right)
$$

where $|\varepsilon| \ll 1$ and $\varepsilon \chi^{(1)}\left(\mathbf{x}^{(0)}\right)$ is a small deformation which in the following will be referred to as the incremental deformation. Note that $\chi^{(1)}$ maps the basic position vector $\mathbf{x}^{(0)}$ into its incremental displacement $\hat{\mathbf{x}}$ in the perturbed actual configuration $\hat{\mathscr{B}}_{a}$. Adopting the convention of summation on repeated indexes, the incremental deformation can be written:

$$
\chi^{(1)}(\mathbf{x})=u_{j}(\mathbf{x}) \mathbf{e}_{j}
$$

where the components $u_{j}(j=1,2,3)$ are the displacements along the principal directions $\mathbf{e}_{j}(j=1,2,3)$, respectively. Figure 10 gives a graphical representation of the basic and perturbed fields. In the following, the variables after perturbation will be indicated with the symbol $(\bar{\bullet})$, and the incremental variables with the symbol $(\hat{\bullet})$. Using the definition of deformation gradient in Eq.(2), the perturbed deformation gradient $\overline{\mathbf{F}}$ rewrites:

$$
\begin{aligned}
\overline{\mathbf{F}}=\mathbf{F}^{(0)}+\varepsilon \hat{\mathbf{F}} & =\frac{\partial \chi^{(0)}(\mathbf{X})}{\partial \mathbf{X}}+\varepsilon \frac{\partial \chi^{(1)}\left(\mathbf{x}^{(0)}\right)}{\partial \mathbf{X}}= \\
& =\frac{\partial \chi^{(0)}\left(\mathbf{x}^{(0)}\right)}{\partial \mathbf{X}}+\varepsilon \frac{\partial \chi^{(1)}\left(\mathbf{x}^{(0)}\right)}{\partial \mathbf{x}^{(0)}} \frac{\partial \mathbf{x}^{(0)}}{\partial \mathbf{X}}=\mathbf{F}^{(0)}+\varepsilon \Gamma \mathbf{F}^{(0)}
\end{aligned}
$$

where the material and the spatial displacement $\operatorname{gradient} \hat{\mathbf{F}}=\operatorname{Grad}\left(\chi^{(1)}\left(\mathbf{x}^{(0)}\right)\right)$ and $\Gamma=\operatorname{grad}\left(\chi^{(1)}\left(\mathbf{x}^{(0)}\right)\right)$ respectively, have been introduced and are related through the following relation:

$$
\hat{\mathbf{F}}=\Gamma \mathbf{F}^{(0)} .
$$

Accordingly, the incremental incompressibility condition in Eq.(92) reads:

$$
\operatorname{tr} \Gamma=0 .
$$

Incremental equilibrium equations Let us now consider the incremental constitutive equation for the stress tensor fields. The perturbed Nominal stress $\overline{\mathbf{S}}$ is given by:

$$
\overline{\mathbf{S}}=\mathbf{S}^{(0)}+\varepsilon \hat{\mathbf{S}},
$$

where the $\mathbf{S}^{(0)}$ is the zeroth order term of the series expansion of the Nominal stress in Eq.(99). The leading order increment $\hat{\mathbf{S}}$ can be derived by differentiating the constitutive equation in Eq. (95) and it reads: 


$$
\hat{\mathbf{S}}=\mathscr{A}^{1} \hat{\mathbf{F}}+p\left(\mathbf{F}^{(0)}\right)^{-1} \hat{\mathbf{F}}\left(\mathbf{F}^{(0)}\right)^{-1}-q\left(\mathbf{F}^{(0)}\right)^{-1} .
$$

In Eq. (110), the term $q=\hat{p}$ is the increment in $p$ and $\mathscr{A}^{1}$ is the fourth-order tensor of the elastic moduli, defined as:

$$
\mathscr{A}^{1}=\frac{\partial \psi_{0}}{\partial \mathbf{F} \partial \mathbf{F}}, \quad \text { with } \quad \mathscr{A}_{\gamma k \beta j}^{1}=\frac{\partial^{2} \psi_{0}}{\partial F_{k \gamma} \partial F_{j \beta}} .
$$

The perturbed Cauchy stress tensor in the perturbed configuration $\hat{\mathscr{B}}_{a}$, is given by $\bar{\sigma}=\overline{\mathbf{F}} \overline{\mathbf{S}}$, but since $\hat{\mathscr{B}}_{a}$ is unknown, it is more practical to use the push-forward of the perturbed Nominal stress, defined as:

$$
\overline{\mathbf{S}}_{0}=\mathbf{F}^{(0)} \overline{\mathbf{S}}=\mathbf{S}_{0}^{(0)}+\varepsilon \hat{\mathbf{S}}_{0}
$$

where, $\mathbf{S}_{0}^{(0)}=\mathbf{F}^{(0)} \mathbf{S}^{(0)}$ is the push forward of the zeroth-order term $\mathbf{S}^{(0)}$. Using Eq.(107) the push-forward $\hat{\mathbf{S}}_{0}$ of the first order term $\hat{\mathbf{S}}$ is given by:

$$
\hat{\mathbf{S}}_{0}=\mathbf{F}^{(0)} \hat{\mathbf{S}}=\mathscr{A}_{0}^{1} \Gamma+p \Gamma-q \mathbf{I}
$$

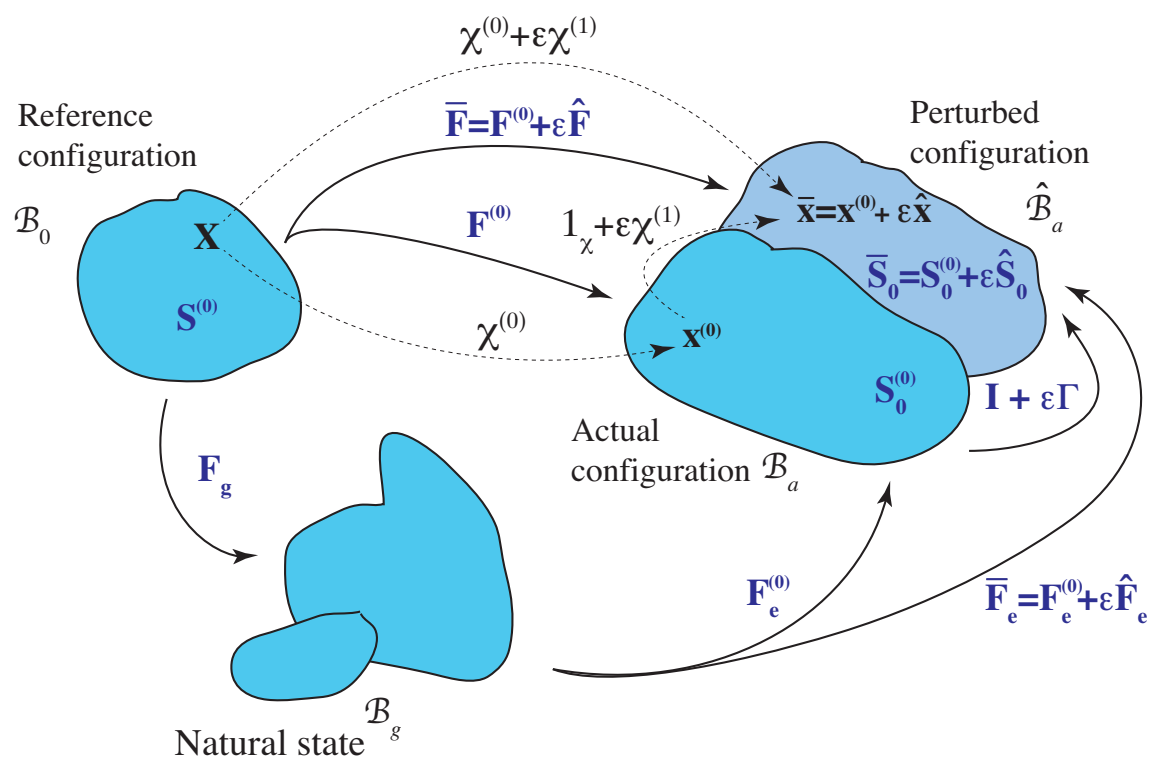

Fig. 10 Schematic diagram of the incremental deformation superposed on a finite strain. The zeroth order fields are the finite deformation $\chi^{(0)}$, the basic position vector $\mathbf{x}^{(0)}$, the basic deformation gradient $\mathbf{F}^{(0)}$ and its elastic component $\mathbf{F}_{e}^{(0)}$, the basic Nominal stress $\mathbf{S}^{(0)}$ and its push-forward $\mathbf{S}_{0}^{(0)}$. The perturbed fields after the introduction of the incremental deformation $\chi^{(1)}$ are the perturbed position vector $\overline{\mathbf{x}}$, the perturbed deformation gradient $\overline{\mathbf{F}}$ and its elastic component $\overline{\mathbf{F}}_{e}$, the push forward of the perturbed Nominal stress $\overline{\mathbf{S}}^{(0)}$. 
with:

$$
\mathscr{A}_{0 h k l j}^{1}=F_{h \gamma}^{(0)} F_{l \beta}^{(0)} \mathscr{A}_{\gamma k \beta j}^{1} .
$$

being the component of the fourth-order tensor $\mathscr{A}_{0}^{1}$, also known as the fourth-order tensor of instantaneous elastic moduli.

Using the properties of the Piola transformation, the incremental equilibrium equations in $\mathscr{B}_{a}$ simplify as:

$$
\operatorname{div} \hat{\mathbf{S}}_{0}=0
$$

where $\hat{\mathbf{S}}_{0}$ is given by Eq.(113).

Let $\hat{\mathbf{u}}_{0}^{*}$ and $\hat{\mathbf{t}}_{0}^{*}$ be the increments of the assigned displacement and traction vectors $\mathbf{u}_{0}^{*}$ and $\mathbf{t}_{0}^{*}$ respectively and $\hat{\mathbf{t}}^{*}$ the increment of the assigned traction vector $\mathbf{t}^{*}$. The Dirichlet boundary conditions in the incremental form rewrite:

$$
\begin{array}{ll}
\hat{\mathbf{x}}(\mathbf{X})=\hat{\mathbf{u}}_{0}^{*} & \text { on } \partial \mathscr{B}_{0}^{\mathbf{x}} \\
\hat{\mathbf{x}}(\mathbf{x})=\chi^{(1)}(\mathbf{x})=\hat{\mathbf{u}}_{0}^{*} & \text { on } \partial \mathscr{B}_{a}^{\mathbf{x}}
\end{array}
$$

and the corresponding incremental boundary conditions for traction loads read:

$$
\begin{array}{ll}
\hat{\mathbf{S}}^{T} \mathbf{N}=\hat{\mathbf{t}}_{0}^{*} & \text { on } \partial \mathscr{B}_{0}^{\sigma} \\
\hat{\mathbf{S}}_{0}^{T} \mathbf{n}=\hat{\mathbf{t}}^{*} & \text { on } \partial \mathscr{B}_{a}^{\sigma} .
\end{array}
$$

We remark that the increment $\hat{\mathbf{t}}^{*}$ in the nominal traction depends in general on the incremental deformation other than on any loading parameter.

\subsubsection{Summary of the incremental boundary value problem}

In summary, the emergence of a morphological transition in living materials provoked by an elastic instability can be studied by solving the following boundary vale problem in nonlinear elasticity:

$$
\left\{\begin{array}{l}
\operatorname{div} \hat{\mathbf{S}}_{0}=0 \\
\operatorname{tr} \Gamma=0
\end{array}\right.
$$

with boundary conditions in the general mixed form:

$$
\hat{\mathbf{x}}(\mathbf{x})=\chi^{(1)}(\mathbf{x})=\hat{\mathbf{u}}_{0}^{*} \text { on } \partial \mathscr{B}_{a}^{\mathbf{x}}, \quad \hat{\mathbf{S}}_{0}^{T} \mathbf{n}=\hat{\mathbf{t}}^{*} \quad \text { on } \partial \mathscr{B}_{a}^{\sigma}
$$

Eqs.(120,121) represent a system of four partial differential equations, where the four unknowns are the three incremental displacements $u_{1}(\mathbf{x}), u_{2}(\mathbf{x}), u_{3}(\mathbf{x})$ in Eq. (105) and the increment $q$ of the Lagrange multiplier. 


\section{Pattern formation in a growing bilayer under lateral constraint}

During embryogenesis, a differential growth between the layers constituting a soft tissue has been found to control the emergence of a pre-patterning, which may drive the later tissue differentiation. For example, it has been shown that both elastic properties and the geometry of the growing layers are key factors determining the pattern selection and the emergence of several functional structures on the epithelium of gastro-intestinal tissues [102, 23].

In the following, we define a morpho-elastic model of a hyperelastic soft tissue made of two flat layers growing under a spatial constraint. Such a bilayered geometry is widely encountered in epithelial tissues, e.g. the dermis and the epidermis for the skin $[75,20]$. Our aim is to investigate the pattern formation in this system model in the absence of differential growth process, simply considering a uniform, homogeneous swelling constrained by the presence of lateral rigid walls.

\subsection{Definition of the model and basic morpho-elastic solution}

In a Cartesian coordinate system with unit base vectors $\mathbf{e}_{i}$, with $i=(x, y, z)$, let us consider a soft elastic tissue attached at the surface $Z=0$, and having a vertical thickness $H$ much smaller than the horizontal lengths $L_{x}, L_{y}$. Moreover, we assume $L_{y}>>L_{x}$ so that we a plain strain condition applies in fulfillment of symmetry requirements. Accordingly, we will consider the equivalent $2 \mathrm{D}$ problem in $(x, z)$ in the following, unless explicitly stated, as depicted in Figure 11.

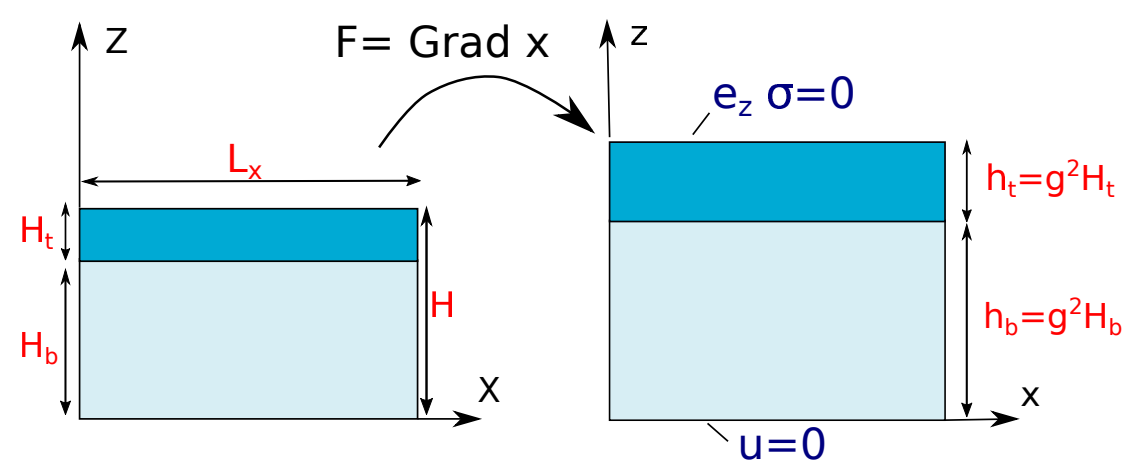

Fig. 11 Schematic diagram of the reference (left) and the basic spatial (right) configurations of a soft bi-layered materials with lateral constraint along the $x$-axis. Both the layers are subjected to a isotropic volumetric growth with $\mathbf{F}_{g}=g \mathbf{I}$, where the scalar $g$ indicates the growth rate.

The tissue is composed by two layers, with thicknesses $H_{b}$ and $H_{t}$, so that $\left(H_{b}+H_{t}\right)=H$. In the following, we will denote with subscripts $b$ and $t$ the fields related to the bottom and top layer, respectively. Indicating with $\mathbf{X}=\mathbf{X}(X, Z)$ and 
$\mathbf{x}=\mathbf{x}(x, z)$ its material and spatial position vectors, respectively, the kinematics is described by the geometrical deformation tensor $\mathbf{F}=\partial \mathbf{x} / \partial \mathbf{X}$. The elastic layers can undergo a isotropic volume variation (e.g. swelling for a polymeric gel, or growth for a living material). Assuming the multiplicative decomposition in Eq.(122), their grown natural states are defined by the growth tensor:

$$
\mathbf{F}_{g}=g \mathbf{I}, \quad \text { with } \mathrm{g}>1
$$

where $g$ is the isotropic growth rate and $\mathbf{I}$ is the unit tensor. Considering the presence on rigid walls at $X=-L_{x} / 2, L_{x} / 2, \mathbf{F}_{g}$ is not compatible with such spatial constraints, therefore an elastic deformation must restores the overall compatibility of the tissue deformation, whilst residual stresses arise.

From a constitutive viewpoint, each layer is modeled as an isotropic neo-Hookean incompressible material, so that strain energy $\Psi_{0}$ per unit mass reads

$$
\Psi_{0}=\frac{\mu}{2} \iint\left(\operatorname{tr} \mathbf{F}_{e} \mathbf{F}_{e}^{T}-3\right) \mathrm{d} X \mathrm{~d} Z
$$

where $\mu$ is the shear modulus.

The base solution for a growing layer, whose fields are indicated with superscript (0) in the following, corresponds to a homogeneous elastic deformation:

$$
\mathbf{F}_{e}^{(0)}=\mathbf{F}^{(0)} \mathbf{F} g^{-1}=\operatorname{diag}\left(\lambda_{x}, \lambda_{z}\right)=\operatorname{diag}(1 / g, g)
$$

whose principal stretches $\lambda_{x}=1 / g$ and $\lambda_{z}=g$ enforce the incompressibility constraint, i.e. $\operatorname{det} \mathbf{F}_{e}^{(0)}=1$. Using the constitutive assumptions in Eq. $(94,95)$, the residual Cauchy stress $\sigma^{(0)}$ and first Piola-Kirchoff stress tensor $\mathbf{P}^{(0)}$ for each layer read:

$$
\sigma^{(0)}=\mu \mathbf{F}_{e}^{(0)} \mathbf{F}_{e}^{(0) T}-p_{0} \mathbf{I}, \quad \mathbf{S}^{(0)}=\mu \mathbf{F}_{e}^{(0)}{ }^{T}-p_{0} \mathbf{F}_{e}^{(0)}{ }^{-1}
$$

where $p_{0}$ is the basic Lagrange multiplier, which can be fixed through the boundary conditions. In particular, considering a stress-free surface at $Z=H$, we have $p_{0 t}=$ $\mu_{t} \lambda_{z}^{2}=\mu_{t} g^{2}$. Moreover, the continuity of the normal stress and displacements at the layers' interface read:

$$
\sigma_{t}^{(0)} e_{z}=\sigma_{b}^{(0)} e_{z} ; \quad \mathbf{x}_{t}\left(x, H_{b}\right)=\mathbf{x}_{b}\left(x, H_{b}\right)
$$

so that $p_{0 b}=\mu_{b} \lambda_{z}^{2}=\mu_{b} g^{2}$. Therefore each layer is subjected to a uniaxial stress with:

$$
\mathbf{S}_{x x}^{(0)}=\mu\left(g^{-1}-g^{3}\right) ; \quad \sigma_{x x}^{(0)}=\mu\left(g^{-2}-g^{2}\right)
$$

which is compressive if $g>1$, i.e. if the tissue is increasing its volume. Therefore, such a uniaxial compressive stress is discountinous within the tissue if the layers have different shear moduli. The goal of the next sextion is to perform a linear stability analysis of this basic solution, with the aim to investigate the condition driving the onset of morphological transition. 


\subsection{Linear stability analysis}

Let us now apply the theory of incremental deformations superposed on the finite homogeneous solution given by Eq.(124). Hence, let $\mathbf{u}^{(1)}(x, z, t)$ be the infinitesimal incremental displacement field. In the case of a neo-Hookean material as in Eq.(123), it is easy to check that the components of the elastic tensor of instantaneous moduli in Eq.(114) read:

$$
\mathscr{A}_{0 j i k l}^{1}=\mu \lambda_{j}^{2} \delta_{j k} \delta_{i l}
$$

where the subscripts run over $(x, z)$ amd $\delta$ is the Kronecker delta. Eq.(128) applies in a coordinate system aligned with the directions of the principal stretches, whilst higher order instantaneous moduli vanish. Accordingly, the neo-hookean constitutive equation allows to take into account only for geometrical non-linearities. From Eq.(113), the constitutive equation for the incremental stress reads:

$$
\hat{\mathbf{S}}_{0}=\mu\left(\tilde{\operatorname{grad}} \mathbf{u}^{(1)}\right)^{T}+p_{0} \operatorname{grad} \mathbf{u}^{(1)}-q \mathbf{I}
$$

where $q$ is the linear increment in the Lagrange multiplier and we defined the operator grad $=\left[\lambda_{x}^{2} \partial_{x}, \lambda_{z}^{2} \partial_{z}\right]^{T}$. In the absence of body forces, the equations of motion in Eq.(115) rewrite:

$$
\operatorname{div} \cdot \hat{\mathbf{S}}_{0}=\mathbf{0}
$$

Using Eq.(129), the incremental equations of motion in Eq.(130) take the following simplified expressions

$$
-q_{, x}+\mu \lambda_{i}^{2} u_{x, i i}^{(1)}=0, \quad-q_{, z}+\mu \lambda_{i}^{2} u_{z, i i}^{(1)}=0,
$$

where the comma denote partial differentiation and Einstein's summation rule on repeated indices is assumed. Differentiating these incremental equations of motion with respect to $x$, and $z$, respectively, and using the incremental incompressibility condition in Eq.(134), I find that

$$
\nabla^{2} q=0
$$

implying that the incremental Lagrange multiplier is a Laplacian field [41].

Conversely, by differentiating the first and second of Eq.(131) by $z$ and $x$, respectively, and subtracting the resulting equations, we find that:

$$
\lambda_{x}^{2} u_{x, z x x}^{(1)}+\lambda_{z}^{2} u_{x, z z z}^{(1)}-\lambda_{z}^{2} u_{z, x z z}^{(1)}-\lambda_{x}^{2} u_{z, x x x}^{(1)}=0,
$$

At the leading order, the linear incremental incompressibility condition rewrites:

$$
\operatorname{div} \cdot \mathbf{u}^{(1)}=u_{x, x}^{(1)}+u_{z, z}^{(1)}=0
$$

The bulk equations $(133,134)$ will be solved in the following using two different methods: the stream function approach and the Stroh formalism. 


\subsubsection{Solution using an elastic stream functions}

First, let us define an elastic stream function $\phi=\phi(x, z)$, so that [91]:

$$
u_{x}^{(1)}=-\phi_{, z}, \quad u_{z}^{(1)}=\phi_{, x},
$$

and Eq.(134) is automatically satisfied. Thus, substituting Eq.(135) in Eq. (133), the incremental equilibrium is governed by the following bulk equation:

$$
\lambda_{z}^{2} \phi_{, z z z z}+\left(\lambda_{x}^{2}+\lambda_{z}^{2}\right) \phi_{, z z x x}+\lambda_{x}^{2} \phi_{, x x x x}=0,
$$

In particular, it is useful to recast the previous equation in the following form [9]:

$$
\nabla^{2} \tilde{\nabla}^{2} \phi=0
$$

where $\tilde{\nabla}^{2}()=.\lambda_{x}^{2}(\cdot)_{, x x}+\lambda_{z}^{2}(.)_{, z z}=(.)_{, X X}+(.)_{, Z Z}$. Accordingly, Eq.(137) states that the equilibrium is governed by a forth order partial differential equation obtained by combination of the material and spatial Laplacian operators. Thus, the growth tensor act as a distortion of the original metrics which changes the differential incremental problem. In the absence of growth, indeed, Eq.(137) would represent the classical bilaplacian problem found for plane strain problems in linear elasticity.

Let us now imposed the required set of boundary conditions. First, since the bottom $z=0$ of the bilayer is attached, the two corresponding incremental boundary conditions read:

$$
\mathbf{u}_{b}^{(1)}(x, 0)=0 .
$$

The top surface $z=\left(\lambda_{z} / \lambda_{x}\right) H=g^{2} H=h$ is free of incremental tractions, thus:

$$
\begin{aligned}
& \left(u_{t}^{(1)}\right)_{x, z}+\left(u_{t}^{(1)}\right)_{z, x}=0, \\
& -\lambda_{z}^{2} q_{t}^{(1)}+2 \mu_{t} \lambda_{z}^{2}\left(u_{t}^{(1)}\right)_{z, z}=0 .
\end{aligned}
$$

Moreover, the four remaining equations will be given by the continuity of the incremental displacements and the normal stresses across the interface at $z=g^{2} H_{b}$, being:

$$
\begin{aligned}
& \mathbf{u}_{b}^{(1)}=\mathbf{u}_{t}^{(1)} \\
& \mu_{t}\left(\left(u_{t}^{(1)}\right)_{x, z}+\left(u_{t}^{(1)}\right)_{z, x}\right)=\mu_{b}\left(\left(u_{b}^{(1)}\right)_{x, z}+\left(u_{b}^{(1)}\right)_{z, x}\right), \\
& -\lambda_{z}^{2} q_{t}^{(1)}+2 \mu_{t} \lambda_{z}^{2}\left(u_{t}^{(1)}\right)_{z, z}=-\lambda_{z}^{2} q_{b}^{(1)}+2 \mu_{b} \lambda_{z}^{2}\left(u_{b}^{(1)}\right)_{z, z} .
\end{aligned}
$$

Let us search for a solution to Eqs.(137) using the variable separation, thus we set:

$$
\phi(x, z)=\Phi(z) e^{\imath k x}+c . c .,
$$

corresponding to the occurrence of a surface undulation with wavenumber $k$ along the $\mathrm{x}$-axis, where $l$ is the imaginary unit and c.c. indicates the complex conjugate. 
the corresponding solution is given by:

$$
\Phi_{i}(z)=a_{1 i} e^{-k z}+a_{2 i} e^{k z}+a_{3 i} e^{-\frac{\lambda_{x}}{\lambda_{z}} k z}+a_{4 i} e^{\frac{\lambda_{x}}{\lambda_{z}} k z} ; \quad \text { with } i=(t, b)
$$

where $a_{1 i}, a_{2 i}, a_{3 i}, a_{4 i}$ are coefficient to be determined by boundary conditions, and we remind that $\lambda_{x} / \lambda_{z}=1 / g^{2}$. Substituting Eq. $(135,142)$ in Eqs.(138,140), the boundary conditions can be transformed in the following way:

$$
\mathbf{M a}=\mathbf{0}
$$

where $\mathbf{a}=\left[a_{1 t}, a_{2 t}, a_{3 t}, a_{4 t}, a_{1 b}, a_{2 b}, a_{3 b}, a_{4 b}\right]^{T}$ and $\mathbf{M}$ is a $8 \times 8$ square matrix, whose detailed expression is given by:

$$
\mathbf{M}=\left[\begin{array}{l}
\mathbf{M}_{1}, \mathbf{M}_{2} \\
\mathbf{M}_{3}, \mathbf{M}_{4}
\end{array}\right]
$$

with sub-blocks given by:

$$
\begin{aligned}
& \mathbf{M}_{1}=\left[\begin{array}{cccc}
0 & 0 & 0 & 0 \\
0 & 0 & 0 & 0 \\
-\frac{e^{-\frac{i k h_{b} \lambda_{x}}{\lambda_{z}}}}{2 k \lambda_{z}^{2} \mu_{t}} & -\frac{e^{\frac{i k h_{b} \lambda_{x}}{\lambda}}}{2 k \lambda_{2}^{2} \mu_{t}} & -\frac{e^{-i k h_{b}}}{k \mu_{t} \lambda_{x}^{2}+k \lambda_{z}^{2} \mu_{t}}-\frac{e^{i k h_{b}}}{k \mu_{t} \lambda_{x}^{2}+k \lambda_{z}^{2} \mu_{t}} \\
\frac{e^{-\frac{i k h_{b} \lambda_{x}}{\lambda_{z}}}}{2 k \lambda_{x} \lambda_{z} \mu_{t}} & -\frac{e^{i k k_{b} \lambda_{x}}}{2 k \lambda_{x} \lambda_{z} \mu_{t}} & \frac{e^{-i k h_{b}}}{k \mu_{t} \lambda_{x}^{2}+k \lambda_{z}^{2} \mu_{t}} & -\frac{e^{i k h_{b}}}{k \mu_{t} \lambda_{x}^{2}+k \lambda_{z}^{2} \mu_{t}}
\end{array}\right],
\end{aligned}
$$

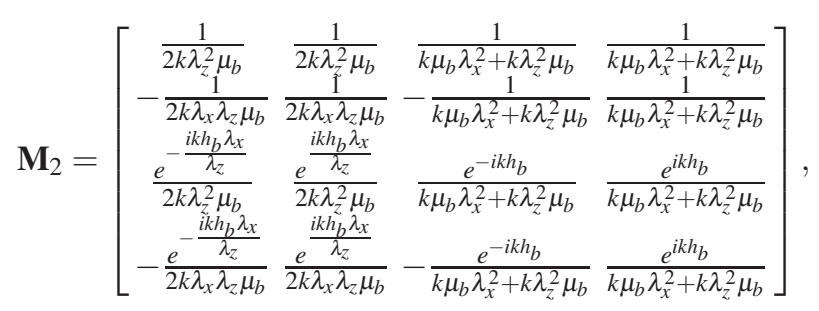

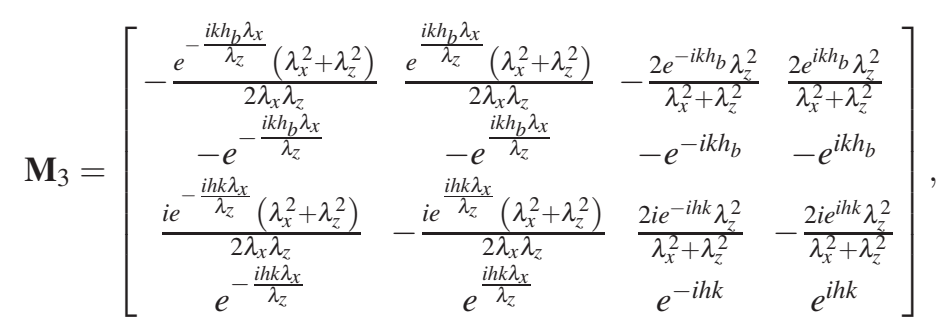

$$
\begin{aligned}
& \mathbf{M}_{4}=\left[\begin{array}{cccc}
\frac{e^{-\frac{i k h_{b} \lambda_{x}}{\lambda_{z}}}\left(\lambda_{x}^{2}+\lambda_{z}^{2}\right)}{2 \lambda_{x} \lambda_{z} \lambda_{z}} & -\frac{e^{\frac{i k h_{b} \lambda_{x}}{\lambda_{z}}}\left(\lambda_{x}^{2}+\lambda_{z}^{2}\right)}{2 \lambda_{x} \lambda_{z}} & \frac{2 e^{-i k h_{b}} \lambda_{z}^{2}}{\lambda_{x}^{2}+\lambda_{z}^{2}} & -\frac{2 e^{i k h_{b} \lambda_{z}^{2}}}{\lambda_{x}^{2}+\lambda_{z}^{2}} \\
e^{-\frac{i k h_{h} \lambda_{x}}{\lambda_{z}}} & e^{\frac{i k_{b} \lambda_{x}}{\lambda_{z}}} & e^{-i k h_{b}} & e^{i k h_{b}} \\
0 & 0 & 0 & 0 \\
0 & 0 & 0 & 0
\end{array}\right] .
\end{aligned}
$$


Accordingly, the onset of a marginal stability is given by the following condition:

$$
\operatorname{det} \mathbf{M}=0
$$

which gives an implicit relation between the marginally stable growth rate $g$ for each wavenumber $k$ as a function of two dimensionless parameters governing the elastic problem, namely the shear ratio $\mu_{t} / \mu_{b}$ and the aspect ratio $H_{t} / H_{b}$.

\subsubsection{Solution using the Stroh formalism}

Let us now solve the incremental boundary value problem recasting the governing equations using the so-called Stroh formalism, originally developed by Stroh [110] for steady state elastic problems. Let the traction vector in the direction $\mathbf{e}_{z}$ be:

$$
\mathbf{t}=\hat{\mathbf{S}}_{0}^{T} \mathbf{e}_{z}=\left[\hat{S}_{0 z x}, \hat{S}_{0 z z}\right]^{T}
$$

In a Cartesian reference system, we can write:

$$
\begin{gathered}
\mathbf{t}=\tilde{\mathbf{A}}_{z z} \mathbf{u}_{, z}+\tilde{\mathbf{A}}_{z x}^{T} \mathbf{u}_{, x}-q \mathbf{e}_{z} \\
\mathbf{t}_{2}=\hat{\mathbf{S}}_{0}^{T} \mathbf{e}_{x}=\tilde{\mathbf{A}}_{z x} \mathbf{u}_{, z}+\tilde{\mathbf{A}}_{x x} \mathbf{u}_{, x}-q \mathbf{e}_{x}
\end{gathered}
$$

where the following matrices have been introduced:

$$
\begin{gathered}
\tilde{\mathbf{A}}_{x x}=\mathscr{A}_{0 z j z l}^{1}+p \mathbf{e}_{z} \otimes \mathbf{e}_{z}, \quad \tilde{\mathbf{A}}_{z z}=\mathscr{A}_{0 x j x l}^{1}+p \mathbf{e}_{x} \otimes \mathbf{e}_{x}, \\
\tilde{\mathbf{A}}_{z x}=\mathscr{A}_{0 z j 1 l}^{1}+p \mathbf{e}_{z} \otimes \mathbf{e}_{x} .
\end{gathered}
$$

The incompressibility condition in Eq.(134) rewrites:

$$
\mathbf{e}_{z} \cdot \mathbf{u}_{, z}+\mathbf{e}_{x} \cdot \mathbf{u}_{, x}=0 .
$$

Substituting Eq.(151) and (152) into Eq.(130), the equilibrium equations rewrite:

$$
\mathbf{t}_{, z}+\tilde{\mathbf{A}}_{z x} \mathbf{u}_{, z x}+\tilde{\mathbf{A}}_{x x} \mathbf{u}_{, x x}-q_{, 2} \mathbf{e}_{x}=0 .
$$

Now, $\mathbf{u}_{, 1}$ can be calculated from Eq.(151) as:

$$
\mathbf{u}_{, 1}=\tilde{\mathbf{A}}_{z z}^{-1}\left(\mathbf{t}-\tilde{\mathbf{A}}_{z x}^{T} \mathbf{u}_{, x}+q \mathbf{e}_{z}\right)
$$

Deriving Eq.(156), it follows:

$$
\begin{gathered}
\mathbf{u}_{, z x}=\tilde{\mathbf{A}}_{z z}^{-1}\left(\mathbf{t}_{, x}-\tilde{\mathbf{A}}_{z x}^{T} \mathbf{u}_{, x x}+q_{, z} \mathbf{e}_{z}\right) \\
\mathbf{u}_{, 13}=\tilde{\mathbf{A}}_{z z}^{-1}\left(\mathbf{t}_{, 3}-\tilde{\mathbf{A}}_{z x}^{T} \mathbf{u}_{, 23}-\tilde{\mathbf{A}}_{31}^{T} \mathbf{u}_{, 33}+q_{, 3} \mathbf{e}_{1}\right)
\end{gathered}
$$

Substituting Eqs.(157) and (158) into Eq.(155), the equilibrium equations in Eq.(155) rewrite: 


$$
\begin{aligned}
\mathbf{t}_{1} & +\tilde{\mathbf{A}}_{z x} \tilde{\mathbf{A}}_{z z}^{-1}\left(\mathbf{t}_{, x}-\tilde{\mathbf{A}}_{z x}^{T} \mathbf{u}_{, x x}+q_{, x} \mathbf{e}_{z}\right)+ \\
& +\tilde{\mathbf{A}}_{x x} \mathbf{u}_{, x x}-q_{, x} \mathbf{e}_{x}=0 .
\end{aligned}
$$

Now, the aim is to eliminate the unknown $q$ from Eq.(159).

Therefore, the scalar product $\mathbf{e}_{1}$. is applied to Eq.(156). Then, using the incompressibility condition in Eq.(154) the term $\mathbf{e}_{1} \cdot \mathbf{u}_{1}$ can be eliminated and after some rearrangements the expression for $q$ can be derived as follows:

$$
q=\xi\left(\mathbf{e}_{1} \cdot \tilde{\mathbf{A}}_{z z}^{-1} \tilde{\mathbf{A}}_{z x}^{T} \mathbf{u}_{, x}-\mathbf{e}_{x} \cdot \mathbf{u}_{, x}-\mathbf{e}_{z} \cdot \tilde{\mathbf{A}}_{z z}^{-1} \mathbf{t}\right),
$$

where $\xi=1 /\left(\mathbf{e}_{z} \cdot \tilde{\mathbf{A}}_{z z}^{-1} \mathbf{e}_{z}\right)$.

Assuming that separation of variables holds, wee search for solutions a in the form:

$$
\begin{aligned}
& \mathbf{u}=\mathbf{U}(z) e^{i k x} \\
& \mathbf{t}=\mathbf{S}(z) e^{i k x},
\end{aligned}
$$

where $k$ is the wavenumber in the $x$ direction, $i$ is the imaginary unit and $\mathbf{U}(z), \mathbf{S}(z)$ are the amplitude of the incremental displacement and traction vectors, respectively. Using Eqs.(160-161), Eq.(156) and Eq.(159) rewrite:

$$
\begin{aligned}
\frac{d}{d z} \mathbf{U}(z)= & -i\left\{\tilde{\mathbf{A}}_{z z}^{-1}\left[\tilde{\mathbf{A}}_{z x}^{T}(k)+\xi \mathbf{e}_{z} \otimes(k \alpha)\right]\right\} \mathbf{U}(z)+ \\
& -i\left\{\tilde{\mathbf{A}}_{z z}^{-1}-\xi \mathbf{e}_{z} \otimes \mathbf{e}_{z} \tilde{\mathbf{A}}_{z z}^{-1}\right\}(i \mathbf{S}(z)) \\
\frac{d}{d z}(i \mathbf{S}(z))= & i\left\{-k^{2}\left[\tilde{\mathbf{A}}_{z x} \tilde{\mathbf{A}}_{z z}^{-1} \tilde{\mathbf{A}}_{z x}^{T}-\tilde{\mathbf{A}}_{x x}-\xi \alpha \otimes \boldsymbol{\alpha}\right]\right\} \mathbf{U}(z)+ \\
& -i\left\{\left[\tilde{\mathbf{A}}_{z x}(k)+\xi k \alpha \otimes \mathbf{e}_{z}\right] \tilde{\mathbf{A}}_{z z}^{-1}\right\}(i \mathbf{S}(z))
\end{aligned}
$$

respectively, where $\alpha=\left(\mathbf{e}_{x}-\tilde{\mathbf{A}}_{z x} \tilde{\mathbf{A}}_{z z}^{-1} \mathbf{e}_{z}\right)$. Equivalently, we can use the compact form:

$$
\frac{d}{d z} \eta(z)=i \mathbf{G} \eta(z) \quad \text { with } \quad \eta(z)=\left[\begin{array}{c}
\mathbf{U}(z) \\
i \mathbf{S}(z)
\end{array}\right]
$$

where the vector $\eta(z)$ is the six-component displacement-traction vector and $\mathbf{G}$ is the so-called Stroh matrix, having the following block-type structure:

$$
\mathbf{G}=\left[\begin{array}{ll}
\mathbf{G}_{1} & \mathbf{G}_{2} \\
\mathbf{G}_{3} & \mathbf{G}_{4}
\end{array}\right]
$$

Therefore, the blocks which constitute the Stroh matrix in the case of incompressible materials take the form:

$$
\begin{aligned}
& \mathbf{G}_{1}=-\tilde{\mathbf{A}}_{z z}^{-1}\left[\tilde{\mathbf{A}}_{z x}^{T}(k)+\xi \mathbf{e}_{z} \otimes(k \alpha)\right] \\
& \mathbf{G}_{2}=-\tilde{\mathbf{A}}_{z z}^{-1}+\xi \mathbf{e}_{z} \otimes \mathbf{e}_{z} \tilde{\mathbf{A}}_{z z}^{-1} \\
& \mathbf{G}_{3}=-k^{2}\left[\tilde{\mathbf{A}}_{z x} \tilde{\mathbf{A}}_{z z}^{-1} \tilde{\mathbf{A}}_{z x}^{T}-\tilde{\mathbf{A}}_{x x}-\xi \alpha \otimes \alpha\right] \\
& \mathbf{G}_{4}=\mathbf{G}_{1}^{+},
\end{aligned}
$$


In particular, the hermitian property $\mathbf{G}_{4}=\mathbf{G}_{1}^{+}$is crucial for the Stroh formalism to provide an optimal form in a great variety of elasticity problems. Optimal here is used in the sense that an efficient numerical procedure can be implemented in order to solve the incremental problem [32, 34, 24]. The hermitian property, also implies that the matrix $i \hat{\mathbf{I}} \mathbf{G}$ is symmetric, where $\hat{\mathbf{I}}$ is defined as the block matrix with 0-blocks on the diagonal and identity blocks elsewhere. This property can be used to derive the orthogonality and closure relations which provide useful information on the nature of the blocks of the Stroh matrix [113]. Furthermore, the hermitian property is a direct consequence of the Hamiltonian nature of the Stroh formalism. Indeed, every Hamiltonian system in which the variables $\mathbf{u}$ and $\mathbf{t}$ are work conjugates owns this fundamental property [42, 43].

In the case of the incremental problem for the growing bilayer, the blocks of the Stroh matrix simplify as follows:

$$
\begin{aligned}
& \mathbf{G}_{1}=\left[\begin{array}{cc}
0 & -m \\
-m & 0
\end{array}\right] ; \quad \mathbf{G}_{2}=\left[\begin{array}{cc}
-1 /\left(\mu \lambda_{z}^{2}\right) & 0 \\
0 & 0
\end{array}\right] ; \\
& \mathbf{G}_{3}=\left[\begin{array}{cc}
k^{2} \mu\left(\lambda_{x}^{2}+3 \lambda_{z}^{2}\right) & 0 \\
0 & k^{2} \mu\left(\lambda_{x}^{2}-\lambda_{z}^{2}\right)
\end{array}\right] .
\end{aligned}
$$

The Stroh formalism allows to transform the boundary value problem into a firstorder system of ordinary differential equation. In particular, its solution is given in terms of displacements as [33]:

$$
\eta_{i}(z)=\sum_{j=1}^{4} a_{j t} \xi_{j} e^{\beta_{j} z} ; \quad \text { with } i=(t, b)
$$

where $\beta=\left\{-k, k,-k \lambda_{x} / \lambda_{z}, k \lambda_{x} / \lambda_{z}\right\}$ and $\xi_{j}$ are the eigenvalues and the $j-t h$ eigenvector $(j=1,2,3,4)$ of $\mathbf{G}$, respectively. Thus, Eq.(168) corresponds to the previous result in Eq.(142) found for with the stream function approach. Similarly, it is possible to recast the eight boundary condition in the form of Eq.(143), thus obtaining the dispersion relation expressed in Eq.(149).

\subsubsection{Theoretical results: critical growth threshold and pattern selection}

We implemented an iterative numerical scheme to solve the dispersion relation in Eq.(149) as a function of the marginal value $\varepsilon=\left(1-\lambda_{x}\right)=(g-1) / g$ for each wavenumber $k=2 \pi n_{x} / L$, with $n_{x}$ integer, at given values of the elastic ratio $\mu_{t} / \mu_{b}$ and aspect ratio $H_{t} / H_{b}$. We therefore identify the critical strain $\varepsilon_{c r}$ as the highest marginally stable value associated to the critical wavelength $k_{c r}$, which will be considered in the following as a continuous function for the sake of graphical clarity. In Figure 12, we depict the corresponding critical threshold for the onset of a morphological transition for a growing bi-layered material with $H_{b}=10 H_{t}$ (blue curves) and $H_{b}=20 H_{t}$ (green curves) as a function of the elastic ratio $\mu_{t} / \mu_{b}$. In particular, we observe that if the top layer is stiffer, the critical strain and the corresponding 

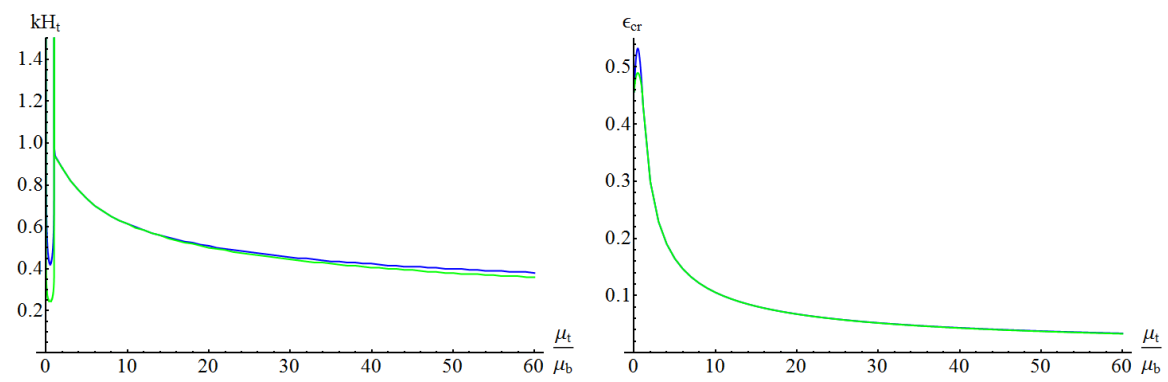

Fig. 12 Curves of the critical dimensionless wavenumber $k_{c r} H_{t}$ (left) and compressive strain $\varepsilon_{c r}=$ $\left(1-\lambda_{x}\right)=(g-1) / g$ (right) for a growing bi-layered material with $H_{b}=10 H_{t}$ (blue curves) and $H_{b}=20 H_{t}$ (green curves) as a function of the elastic ratio $\mu_{t} / \mu_{b}$.

wavenumber decrease for increasing $\mu_{t} / \mu_{b}$. Moreover, we find that the emerging pattern wavelength is of the order of the thickness of the top layer, whilst the results do not depend much on the aspect ration if $H_{t}<<H_{b}$.

We also notice that the marginally stable curve for $k_{c r} H_{t}$ has a singular behaviour for $\mu_{t} / \mu_{b} \rightarrow 1$ and $\mu_{t} / \mu_{b} \rightarrow 0$. Both cases represent the confined compression of a homogeneous slab, which is know to experience a surface instability at $k_{c r} \rightarrow \infty$, as first predicted for an elastic half-space by Biot [11]. In this case, the dispersion relation in Eq.(149) can be greatly simplified, and its marginally stable solution occurs at $k_{c r} \rightarrow \infty$ for the real root of $\left(\lambda_{x}^{6}+\lambda_{x}^{4}+3 \lambda_{x}^{2}-1\right)=0$, being:

$$
\left(\lambda_{x}\right)_{c r}=\frac{1}{3}\left(\sqrt[3]{17+3 \sqrt{33}}-\frac{2}{\sqrt[3]{17+3 \sqrt{33}}}-1\right) \cong 0.5436
$$

implying a critical growth ratio of $g_{c r} \cong 1.8393$ or a critical strain of $\varepsilon_{c r} \cong 0.4563$, as depicted in greated detail in Figure 13.
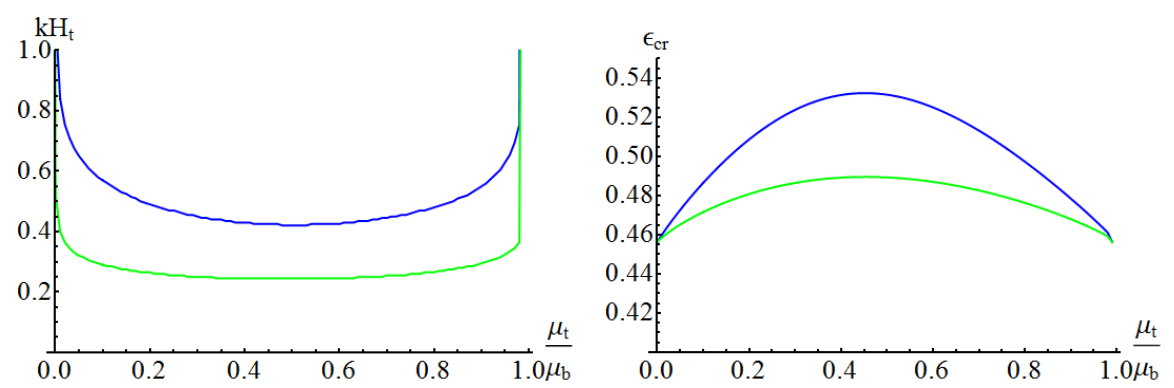

Fig. 13 Zoom of the marginally stable curves for the critical dimensionless wavenumber $k_{c r} H_{t}$ (left) and compressive strain $\varepsilon_{c r}=\left(1-\lambda_{x}\right)=(g-1) / g$ (right) for a growing bi-layered material with $H_{b}=10 H_{t}$ (blue curves) and $H_{b}=20 H_{t}$ (green curves) as a function of the elastic ratio $\mu_{t} / \mu_{b}$. 
It is also possible to show that the critical wavenumbers in such singular branches have a logarithmic dependence on the surface stiffening effect given by the top layer $[9,20]$.

From an energetic viewpoint, the onset of a morphological transition is driven by a competition between the two bulk elastic energies, through a complex interplay between geometric and elastic parameters. The linear stability analysis allows to identify the critical threshold of such a bifurcation, but it cannot describe its evolution beyond this threshold value. For this purpose, it would be necessary to solve the incremental problem beyond the linear approximation. Although out of the scopes of this chapter, we highlight that a weakly nonlinear stability analysis could be performed using a multiple scale expansion [19]. Accordingly, it is possible to derive a compatibility condition by imposing the vanishing of secular terms in higher-order elastic solutions, thus unveiling the nature of the elastic bifurcation [25]. For example, if the bottom layer can be seen as an elastic halfspace, i.e. assuming $H_{b}<<H_{t}$, it can be shown that the elastic bifurcation is supercritical if $\mu_{t} / \mu_{b}>1.74$ and subcritical if $\mu_{t} / \mu_{b}<1.74$ [16]; whilst in the subcritical regime for $\mu_{t} \rightarrow \mu_{b}$ there exists a localized solution that arises as the limit of modulated periodic solutions with increasingly longer and longer decaying tails. The evolution of each modulated periodic solution can be followed as $\mu_{t} / \mu_{b}$ is decreased,finding that there exists a critical value at which the deformation gradient develops a discontinuity and the solution becomes a static shock, highlighting the spontaneous formation of a crease [45].

\subsubsection{Numerical results: post-buckling behavior}

In order to explore the behavior of the system in the post-critical regime, we implemented numerical simulations using a user defined finite element (FE) code implemented in Matlab (The MathWorks, Inc., Natick, Massachusetts, United States; version 2013a). The geometrical model is composed by two layers, a top layer superposed on a substrate with initial thicknesses $H_{t}$ and $H_{b}$, respectively. The bi-layer has initial width $L_{x}$. Since the value of $k$ is predicted by the linear stability analysis, $L_{x}$ can be calculated at $n_{x}$ arbitrarily fixed. The two layers are modeled as incompressible and hyperelastic materials. A Neo-Hookean model is used, and boundary conditions of zero-displacement at the the bottom of the bi-layer and zero-horizontal displacement at the side edges are imposed.

We model the growth using a pseudo-dynamic method similar to the one classically implemented for thermal dilatation. We adopt the following linear evolution law:

$$
g(t)=g\left(t_{n-1}\right) d t
$$

with $g(0)=1$ and where $t$ is the time and $d t=\left(t_{n}-t_{n-1}\right)$ is the time increment. An ad hoc time adaptive scheme is implemented, where the time step automatically decreases by $50 \%$ once the number of Newton iterations exceeds seven, and increases the time step size by $10 \%$ otherwise, as done in [92]. The initial, the minimum and the maximum time steps are manually set to $10^{2}, 10^{3}$ and $10^{2}$, respectively. A si- 
nusoidal perturbation is introduced as a small imperfection in the initial mesh, with a small amplitude of $0.005 H_{t}$ and the critical wavenumber predicted by the linear stability analysis.

We used linear elements with 4 integration points, building the mesh with 400 elements in the $\mathrm{x}$-direction and 150 elements in the z-direction, respectively 100 elements for the substrate and 50 for the film.

Validation of the numerical code against the theoretical results First, we validate the FE code by comparing the critical strain values in numerical simulation with the theoretical predictions from the linear stability analysis. The criterion for such a comparison is based on the energy considerations, since we expect that the selected pattern will minimizes the total elastic energy of the system. Before the onset of the pattern transition, the solution with the minimal elastic energy is the basic homogeneous deformation. Due to the spatial incompatibility of the growth process, residual stresses accumulate until a marginally stable growth value is reached. At this point, the system bifurcates into a solution with a lower energy than the one associated with the basic homogeneous solution.

Here we identify the critical strain as the value of $\varepsilon_{c r}=\left(1-\lambda_{x}\right)=(g-1) / g$ at which the ratio $E / E_{0}$ between the total current elastic energy and the basic elastic energy has decreased by more than $1 \%$ of its initial value. The corresponding energy plots are depicted in Figure 14(left) at different values of the elastic ratio $\mu_{t} / \mu_{b}$.
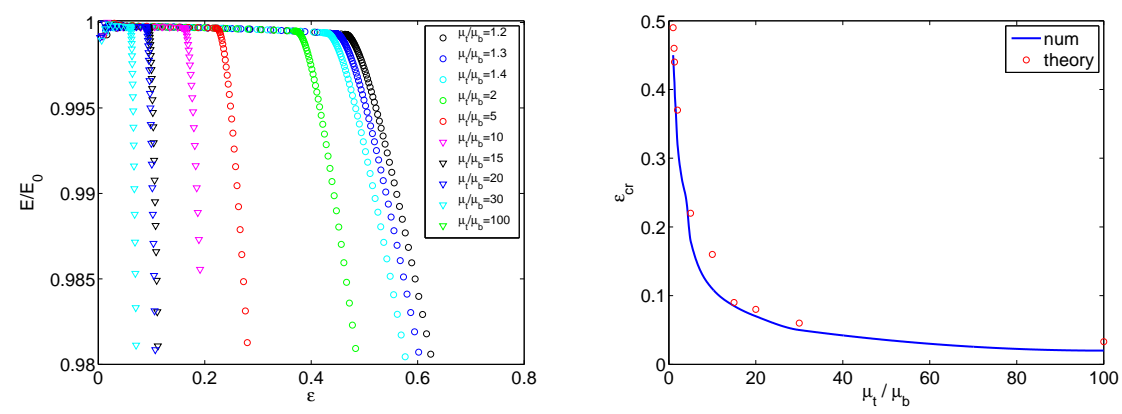

Fig. 14 Left: Ratio between the total current elastic energy in the numerical simulations $E$ and the elastic energy of the basic homogeneous solution $E_{0}$ versus the axial strain $\varepsilon=\left(1-\lambda_{x}\right)$ at different values of the elastic ratio $\mu_{t} / \mu_{b}$. Right: Comparison between the marginally stable thresholds in numerical FE simulations (circles) and the theoretical predictions (solid line) from the linear stability analysis. We set $H_{b}=10 H_{t}$ in all the simulations.

In particular, Figure 14 (right) shown that the critical values resulting from the numerical simulations are in good agreement with the corresponding theoretical prediction, thus validating the numerical code.

FE simulations of the post-critical regime Once the FE code has been validated, we performed numerical simulations in order to investigate the nonlinear pattern 
evolution in the post-critical regime. We depict the emerging morphology of the growing bi-layer for $\mu_{t} / \mu_{b}=30,100$ in Figures 15,16 respectively.
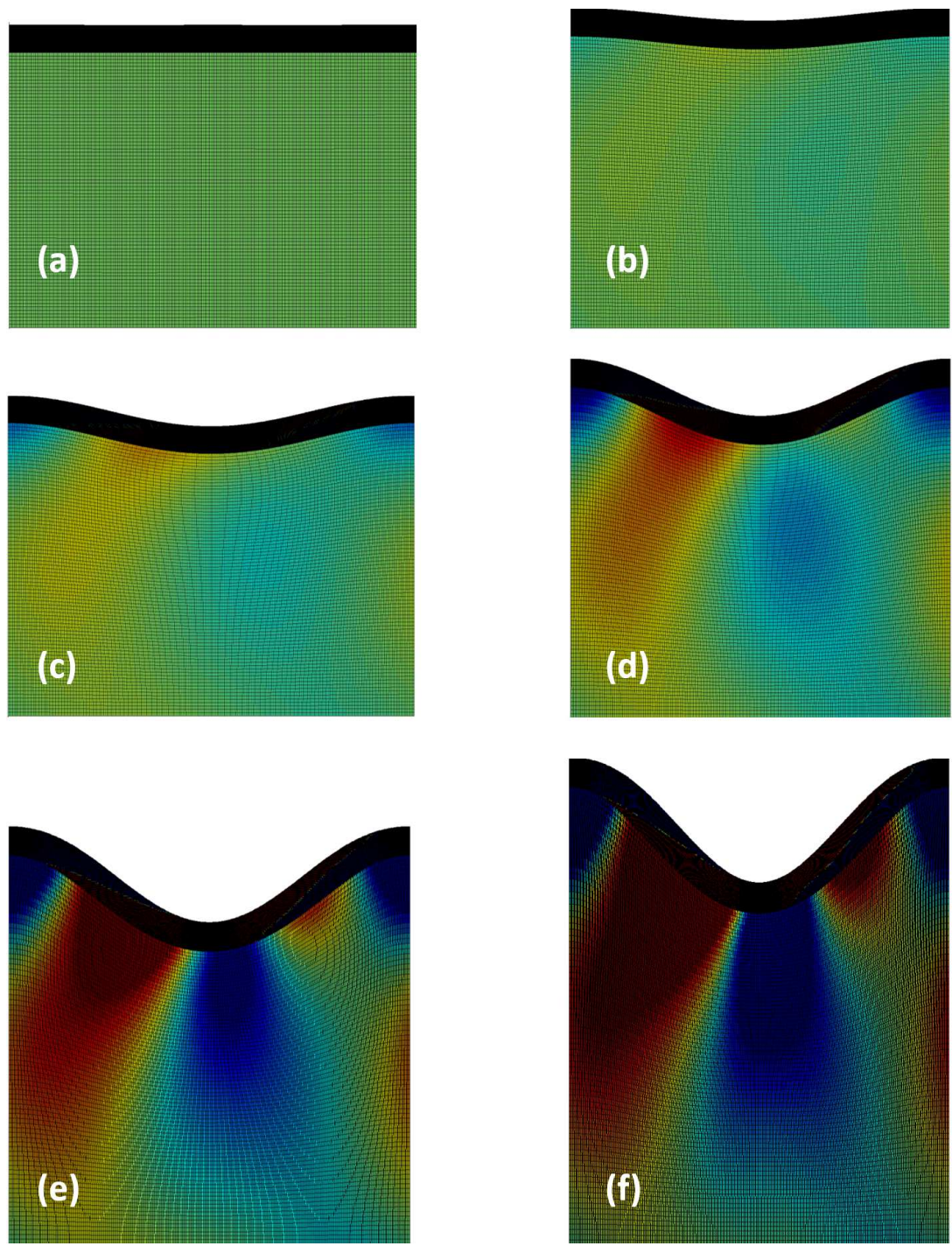

Fig. 15 Morphological evolution of the bi-layered material at different growth ratios. We set $\mu_{t} / \mu_{b}=30, H_{b}=10 H_{t}, n_{x}=1$, for which the linear stability analysis predicts $k^{c r}=0.4, \varepsilon^{c r}=0.05$. The snapshots are taken for $\varepsilon=(g-1) / g=0$ (a), 0.063004 (b), 0.075409 (c), 0.108321 (d), 0.165309 (e), 0.283784 (f). 
(a)

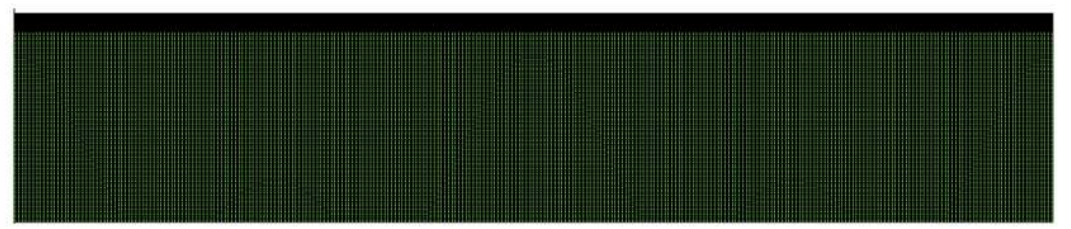

(b)

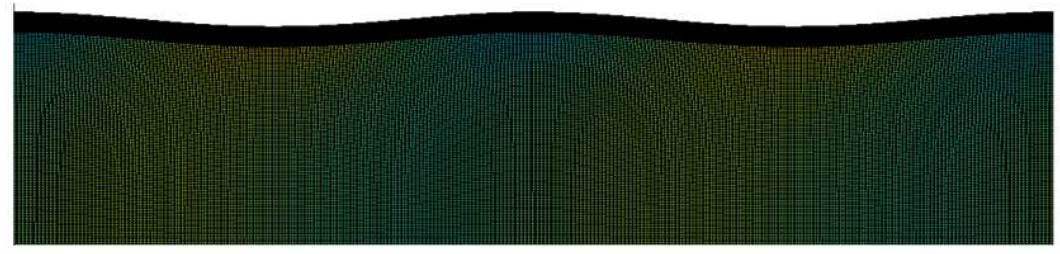

(c)

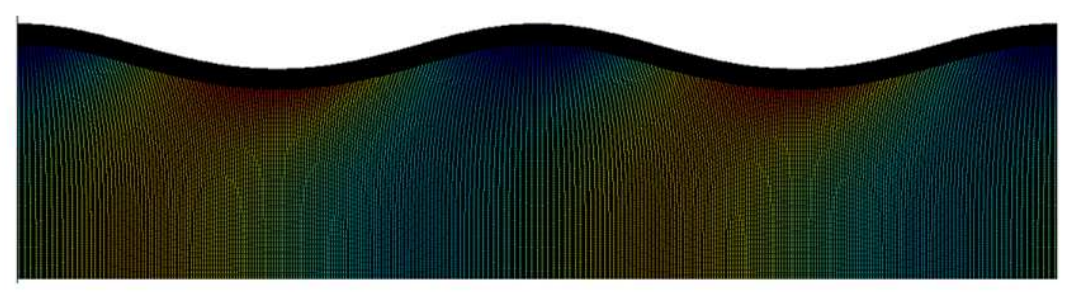

\section{(d)}

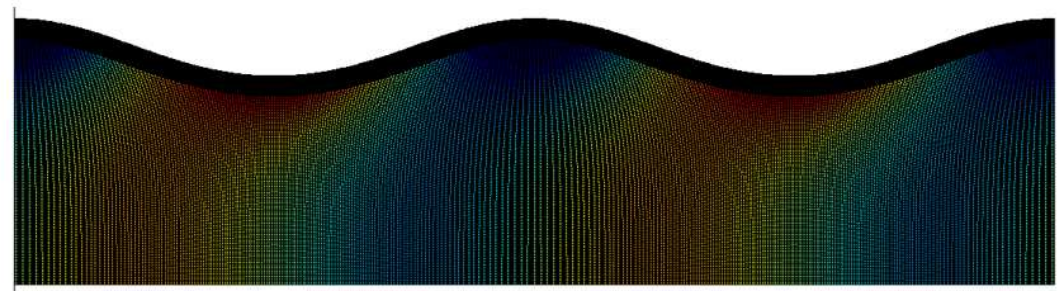

Fig. 16 Morphological evolution of the bi-layered material at different growth ratios. We set $\mu_{t} / \mu_{b}=100, H_{b}=10 H_{t}, n_{x}=2$, for which the linear stability analysis predicts $k^{c r}=0.23$, $\varepsilon^{c r}=0.02$. The snapshots are taken for $\varepsilon=(g-1) / g=0$ (a), 0.033173 (b), 0.042321 (c), 0.059083 (d).

Before the critical point is reached, the bi-layered material remains flat. As soon as the compression reaches the critical value, an undulated pattern develops at the top surface, forming wrinkles with wavenumber $k_{c r}$. The depth of the wrinkles in- 
creases whilst increasing the growth, which increases the axial compression. In both cases we set $H_{b}=10 H_{t}$. The critical values for the wavenumber and the compression given by the linear stability analysis are $k^{c r}=0.4, \varepsilon^{c r}=0.05$ for $\mu_{t} / \mu_{b}=30$, and $k^{c r}=0.23, \varepsilon^{c r}=0.02$ for $\mu_{t} / \mu_{b}=100$.

In practice, we observe that the surface undulation rapidly grows to form a stable fold, whose amplitude increases whilst increasing the growth ratio. The amplitude of the emerging surface fold is depicted in Figure 17 for different values of the elastic ratio $\mu_{t} / \mu_{b}$. In particular, we show that for $\mu_{t}>>\mu_{b}$ the amplitude grows continu-

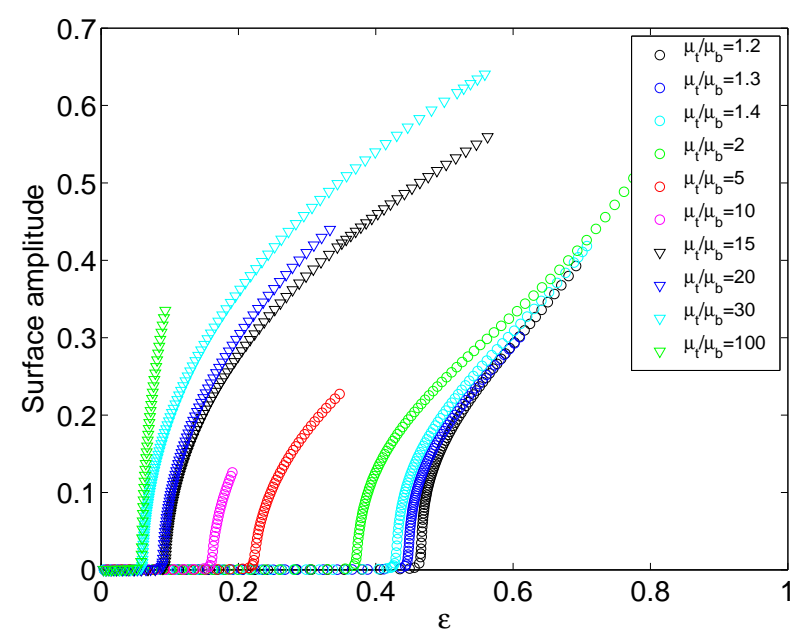

Fig. 17 Amplitude of the surface folds in numerical simulations for different values of the elastic ratio $\mu_{t} / \mu_{b}$ at $H_{b}=10 H_{t}$.

ously, thus highlighting the existence of a supercritical bifurcation, as predicted for the coated elastic half-space.

It is important to highlight that we used linear elements for illustrative purposes. Nonetheless, it is known that they do not allow to capture the occurrence of secondary bifurcations, which have been observed in bi-layered materials, possibly leading to the emergence of more complex patterns characterized by sub-harmonic resonances [14]. Although out of the scopes of this work, we remind that secondary bifurcations can be studied using analytic perturbation techniques [44] or more advances numerical tools [81]. 


\section{Concluding remarks}

In this chapter, we have introduced a continuous chemo-mechanical approach of morphogenesis, deriving the balance principles and evolution laws for both volumetric and interfacial processes. The proposed theory has been applied to the study of pattern formation for either a fluid-like and a solid-like biological system model, using both theoretical methods and simulation tools. Nonetheless, it should be reminded that biological materials have a wide range of rheological properties in between such limiting ideal behaviors [115]. Furthermore, it has been recently highlighted that morphogenetic processes may involve microstructural rearrangement processes, such cell duplication and/or migration, which provoke fluid-like stress relaxation phenomena up to the timescale of days $[96,97]$. Thus, there will be the necessity to integrate the continuous approach with methods of individual cell-based models in order to capture the microscopic processes at the grain cell scale [35].

Finally, further modelling work should be done to investigate the role of morphogens in the regulation of growth, shape and size. On one hand, their local concentration can trigger an increase of mass resulting from a random cellular proliferation. Preferred orientation may exists in cellular division as well as competition between different cell populations. On the other hand, further spatial orchestration is needed in order to transform random proliferations into a uniform growth, which must ultimately ceases as the correct size is reached. The control of shape can therefore depend both on cell number and on overall size, suggesting that the spatial gradient of morphogens can provide cells a dimension-sensing mechanism. This early vision was also supported by the discovery that cells have the ability to measure gradients comparing their own signalling level with those of their neighbours through specific regulatory pathways [125]. Nevertheless other interpretations are also possible. In fact, cells can change relative position undergoing a rearrangement process (e.g. during intercalation), implying that they are able to remodel their adhesive contacts making use of a mechanical feedback with their environment to adjust their position [77]. Giving a practical example, the stop signal for growth, thus determining the final size, could be triggered by a morphogen gradient level below a minimal threshold, as well as by a critical increase in tissue compression, causing a progressive inhibition of growth [5]. In summary, the orchestration of shape and size in the biological realm is more likely based on a combination of mechanical and biochemical feedbacks. Despite of the explosive rate of new knowledge on the biochemistry of morphogenesis, a major challenge for mathematical modeling is to understand the local coordination between mechanical properties of the cells and the morphogenetic signals for the global orchestration of a macroscopic shape[109].

Acknowledgements We are thankful to Chiara Giverso for performing the numerical simulations shown in Figure 8, and to Ellen Kuhl for sharing the numerical FE code used in Section 6.2.4. Partial funding by the European Community grant ERG-256605, FP7 program, and by the "Startup Packages and PhD Program" project, co-funded by Regione Lombardia through the "Fondo per lo sviluppo e la coesione 2007-2013", formerly FAS program, is gratefully acknowledged by the corresponding author. 


\section{References}

[1] H. B. Adelmann, M. Malpighi, et al. Marcello malpighi and the evolution of embryology. 1966.

[2] P. Alberch. Developmental constraints in evolutionary processes. In Evolution and development, pages 313-332. Springer, 1982.

[3] J. O. Almen and P. H. Black. Residual stresses and fatigue in metals. McGraw-Hill, 1963.

[4] D. Ambrosi and F. Mollica. On the mechanics of a growing tumor. International journal of engineering science, 40(12):1297-1316, 2002.

[5] D. Ambrosi, V. Pettinati, and P. Ciarletta. Active stress as a local regulator of global size in morphogenesis. International Journal of Non-Linear Mechanics, 2014.

[6] D. Balme. Aristotle. history of animals. books vii-x, 1991.

[7] J. B. Bard and J. Bard. Morphogenesis: the cellular and molecular processes of developmental anatomy, volume 23. Cambridge University Press, 1992.

[8] L. Beloussov. The interplay of active forces and passive mechanical stresses in animal morphogenesis. In Biomechanics of Active Movement and Division of Cells, pages 131-180. Springer, 1994.

[9] M. Ben Amar and P. Ciarletta. Swelling instability of surface-attached gels as a model of soft tissue growth under geometric constraints. Journal of the Mechanics and Physics of Solids, 58(7):935-954, 2010.

[10] C. Bernard and É. Alglave. Leçons sur les propriétés des tissus vivants. Baillière, 1866.

[11] M. Biot. Surface instability of rubber in compression. Applied Scientific Research, Section A, 12(2):168-182, 1963.

[12] A. Boettiger, B. Ermentrout, and G. Oster. The neural origins of shell structure and pattern in aquatic mollusks. Proceedings of the National Academy of Sciences, 106(16):6837-6842, 2009.

[13] E. Buckingham. On physically similar systems; illustrations of the use of dimensional equations. Physical Review, 4(4):345-376, 1914.

[14] S. Budday, E. Kuhl, and J. W. Hutchinson. Period-doubling and periodtripling in growing bilayered systems. Philosophical Magazine, (ahead-ofprint): 1-17, 2015.

[15] D. T. Butcher, T. Alliston, and V. M. Weaver. A tense situation: forcing tumour progression. Nat Rev Cancer, 9(2):108-122, 2009.

[16] Z. Cai and Y. Fu. On the imperfection sensitivity of a coated elastic halfspace. In Proceedings of the Royal Society of London A: Mathematical, Physical and Engineering Sciences, volume 455, pages 3285-3309. The Royal Society, 1999.

[17] C. Chuong and Y. Fung. Residual stress in arteries. In Frontiers in Biomechanics, pages 117-129. Springer, 1986.

[18] P. Ciarletta. Free boundary morphogenesis in living matter. European Biophysics Journal, 41(8):681-686, 2012. 
[19] P. Ciarletta. Wrinkle-to-fold transition in soft layers under equi-biaxial strain: A weakly nonlinear analysis. Journal of the Mechanics and Physics of Solids, 73:118-133, 2014.

[20] P. Ciarletta and M. B. Amar. Papillary networks in the dermal-epidermal junction of skin: a biomechanical model. Mechanics Research Communications, 42:68-76, 2012.

[21] P. Ciarletta, D. Ambrosi, and G. Maugin. Configurational forces for growth and shape regulations in morphogenesis. Bulletin of the Polish Academy of Sciences: Technical Sciences, 60(2):253-257, 2012.

[22] P. Ciarletta, D. Ambrosi, and G. Maugin. Mass transport in morphogenetic processes: a second gradient theory for volumetric growth and material remodeling. Journal of the Mechanics and Physics of Solids, 60(3):432-450, 2012.

[23] P. Ciarletta, V. Balbi, and E. Kuhl. Pattern selection in growing tubular tissues. Physical review letters, 113(24):248101, 2014.

[24] P. Ciarletta and M. Destrade. Torsion instability of soft solid cylinders. IMA Journal of Applied Mathematics, 79(5):804-819, 2014.

[25] P. Ciarletta and Y. Fu. A semi-analytical approach to biot instability in a growing layer: Strain gradient correction, weakly non-linear analysis and imperfection sensitivity. International Journal of Non-Linear Mechanics, 2015.

[26] P. Ciarletta and G. A. Maugin. Elements of a finite strain-gradient thermomechanical theory for material growth and remodeling. International Journal of Non-Linear Mechanics, 46(10):1341-1346, 2011.

[27] P. Ciarletta, L. Preziosi, and G. Maugin. Thermo-mechanics of growth and mass transfer: morphogenesis of seashells. Computer methods in biomechanics and biomedical engineering, 15(sup1):110-112, 2012.

[28] P. Ciarletta, L. Preziosi, and G. Maugin. Mechanobiology of interfacial growth. Journal of the Mechanics and Physics of Solids, 61(3):852-872, 2013.

[29] C. Cohen. Gould et d'arcy thompson. Comptes Rendus Palevol, 3(5):421431, 2004.

[30] B. D. Coleman and W. Noll. The thermodynamics of elastic materials with heat conduction and viscosity. Archive for Rational Mechanics and Analysis, 13(1):167-178, 1963.

[31] J. Davies. Mechanisms of morphogenesis. Academic Press, 2013.

[32] M. Destrade, A. N. Annaidh, and C. D. Coman. Bending instabilities of soft biological tissues. International Journal of Solids and Structures, 46(25):4322-4330, 2009.

[33] M. Destrade, M. Gilchrist, D. Prikazchikov, and G. Saccomandi. Surface instability of sheared soft tissues. Journal of biomechanical engineering, 130(6):061007, 2008.

[34] M. Destrade, J. G. Murphy, and R. W. Ogden. On deforming a sector of a circular cylindrical tube into an intact tube: existence, uniqueness, and stability. International Journal of Engineering Science, 48(11):1212-1224, 2010. 
[35] D. Drasdo, S. Hoehme, and M. Block. On the role of physics in the growth and pattern formation of multi-cellular systems: What can we learn from individual-cell based models. J Stat Phys, 128(1-2):287-345, 2007.

[36] W. Driever and C. Nüsslein-Volhard. The bicoid protein determines position in the drosophila embryo in a concentration-dependent manner. Cell, 54(1):95-104, 1988.

[37] C. C. DuFort, M. J. Paszek, and V. M. Weaver. Balancing forces: architectural control of mechanotransduction. Nature reviews Molecular cell biology, 12(5):308-319, 2011.

[38] G. M. Edelman. Morphoregulatory molecules. Biochemistry, 27(10):35333543, 1988.

[39] M. Epstein and A. Goriely. Self-diffusion in remodeling and growth. Zeitschrift für angewandte Mathematik und Physik, 63(2):339-355, 2012.

[40] M. Epstein and G. A. Maugin. Thermomechanics of volumetric growth in uniform bodies. International Journal of Plasticity, 16(7):951-978, 2000.

[41] J. Flavin. Surface waves in pre-stressed mooney material. The Quarterly Journal of Mechanics and Applied Mathematics, 16(4):441-449, 1963.

[42] Y. Fu. Existence and uniqueness of edge waves in a generally anisotropic elastic plate. The Quarterly Journal of Mechanics and Applied Mathematics, 56(4):605-616, 2003.

[43] Y. Fu and D. Brookes. Edge waves in asymmetrically laminated plates. Journal of the Mechanics and Physics of Solids, 54(1):1-21, 2006.

[44] Y. Fu and Z. Cai. An asymptotic analysis of the period-doubling secondary bifurcation in a film/substrate bilayer. SIAM Journal on Applied Mathematics, 75(6):2381-2395, 2015.

[45] Y. Fu and P. Ciarletta. Buckling of a coated elastic half-space when the coating and substrate have similar material properties. In Proceedings of the Royal Society of London A: Mathematical, Physical and Engineering Sciences, volume 471, page 20140979. The Royal Society, 2015.

[46] Y.-C. Fung. Biomechanics: mechanical properties of living tissues. Springer Science \& Business Media, 2013.

[47] G. Galilei. Discorsi e dimostrazioni matematiche intorno a due nuove scienze. Elzeviro, 1638.

[48] A. Gierer and H. Meinhardt. A theory of biological pattern formation. Kybernetik, 12(1):30-39, 1972.

[49] C. Giverso, M. Verani, and P. Ciarletta. Branching instability in expanding bacterial colonies. Journal of The Royal Society Interface, 12(104):20141290, 2015.

[50] A. Goriely and M. B. Amar. On the definition and modeling of incremental, cumulative, and continuous growth laws in morphoelasticity. Biomechanics and modeling in mechanobiology, 6(5):289-296, 2007.

[51] S. J. Gould. Ontogeny and phylogeny. Harvard University Press, 1977.

[52] J. B. Green and J. Sharpe. Positional information and reaction-diffusion: two big ideas in developmental biology combine. Development, 142(7):12031211, 2015. 
[53] H. Gregersen, G. Kassab, and Y. Fung. Review: The zero-stress state of the gastrointestinal tract. Digestive diseases and sciences, 45(12):2271-2281, 2000.

[54] E. Guyon, J.-P. Hulin, L. Petit, and P. G. de Gennes. Hydrodynamique physique. EDP sciences Les Ulis, France, 2001.

[55] E. H. Haeckel. Generelle Morphologie der Organismen allgemeine Grundzuge der organischen Formen-Wissenschaft, mechanisch begrundet durch die von Charles Darwin reformirte Descendenz-Theorie von Ernst Haeckel: Allgemeine Entwickelungsgeschichte der Organismen kritische Grundzuge der mechanischen Wissenschaft von den entstehenden Formen der Organismen, begrundet durch die Descendenz-Theorie, volume 2. Verlag von Georg Reimer, 1866.

[56] V. Hamburger. Wilhelm roux: visionary with a blind spot. Journal of the History of Biology, 30(2):229-238, 1997.

[57] H. Han and Y. Fung. Residual strains in porcine and canine trachea. Journal of biomechanics, 24(5):307-315, 1991.

[58] M. P. Harris, S. Williamson, J. F. Fallon, H. Meinhardt, and R. O. Prum. Molecular evidence for an activator-inhibitor mechanism in development of embryonic feather branching. Proceedings of the National Academy of Sciences of the United States of America, 102(33):11734-11739, 2005.

[59] N. Hartsoeker. Conjectures physiques, volume 2. 1708.

[60] W. Harvey and R. Willis. The works of William Harvey, volume 11. Sydenham society, 1847.

[61] B. D. Hoffman, C. Grashoff, and M. A. Schwartz. Dynamic molecular processes mediate cellular mechanotransduction. Nature, 475(7356):316-323, 2011.

[62] A. Hoger. On the residual stress possible in an elastic body with material symmetry. Archive for Rational Mechanics and Analysis, 88(3):271-289, 1985.

[63] A. Hoger. On the determination of residual stress in an elastic body. Journal of Elasticity, 16(3):303-324, 1986.

[64] A. Hoger. Residual stress in an elastic body: a theory for small strains and arbitrary rotations. Journal of Elasticity, 31(1):1-24, 1993.

[65] A. Hoger. The elasticity tensor of a transversely isotropic hyperelastic material with residual stress. Journal of Elasticity, 42(2):115-132, 1996.

[66] J. Howard, S. W. Grill, and J. S. Bois. Turing's next steps: the mechanochemical basis of morphogenesis. Nature Reviews Molecular Cell Biology, 12(6):392-398, 2011.

[67] J. S. Huxley. 1932problems of relative growth. London: Methuen.

[68] J. Jaeger, D. Irons, and N. Monk. Regulative feedback in pattern formation: towards a general relativistic theory of positional information. Development, 135(19):3175-3183, 2008.

[69] J. Jaeger, S. Surkova, M. Blagov, H. Janssens, D. Kosman, K. N. Kozlov, E. Myasnikova, C. E. Vanario-Alonso, M. Samsonova, D. H. Sharp, et al. 
Dynamic control of positional information in the early drosophila embryo. Nature, 430(6997):368-371, 2004.

[70] B. E. Johnson and A. Hoger. The dependence of the elasticity tensor on residual stress. Journal of Elasticity, 33(2):145-165, 1993.

[71] E. F. Keller and L. A. Segel. Model for chemotaxis. Journal of theoretical biology, 30(2):225-234, 1971.

[72] S. Kondo and R. Asai. A reaction-diffusion wave on the skin of the marine angelfish pomacanthus. Nature, 376(6543):765-768, 1995.

[73] S. Kondo and T. Miura. Reaction-diffusion model as a framework for understanding biological pattern formation. science, 329(5999):1616-1620, 2010.

[74] E. Kröner. Allgemeine kontinuumstheorie der versetzungen und eigenspannungen. Arch Ration Mech An, 4(1):273-334, 1959.

[75] M. Kücken and A. C. Newell. Fingerprint formation. Journal of theoretical biology, 235(1):71-83, 2005.

[76] T. Lecuit and P.-F. Lenne. Cell surface mechanics and the control of cell shape, tissue patterns and morphogenesis. Nature Reviews Molecular Cell Biology, 8(8):633-644, 2007.

[77] T. Lecuit and P.-F. Lenne. Cell surface mechanics and the control of cell shape, tissue patterns and morphogenesis. Nature Rev Mol Cell Biol, 8(8):633-644, 2007.

[78] T. Lecuit, P.-F. Lenne, and E. Munro. Force generation, transmission, and integration during cell and tissue morphogenesis. Annual review of cell and developmental biology, 27:157-184, 2011.

[79] E. H. Lee. Elastic-plastic deformation at finite strains. Journal of Applied Mechanics, 36(1):1-6, 1969.

[80] H. Levine, S. Pamuk, B. Sleeman, and M. Nilsen-Hamilton. Mathematical modeling of capillary formation and development in tumor angiogenesis. Evolution, 2012, 2011.

[81] J. Liu and K. Bertoldi. Bloch wave approach for the analysis of sequential bifurcations in bilayer structures. In Proc. R. Soc. A, volume 471, page 20150493. The Royal Society, 2015.

[82] P. K. Maini, D. S. McElwain, and D. I. Leavesley. Traveling wave model to interpret a wound-healing cell migration assay for human peritoneal mesothelial cells. Tissue engineering, 10(3-4):475-482, 2004.

[83] G. Maugin. On inhomogeneity, growth, ageing and the dynamics of materials. Journal of Mechanics of Materials and Structures, 4(4):731-741, 2009.

[84] G. Maugin and C. Trimarco. Pseudomomentum and material forces in nonlinear elasticity: variational formulations and application to brittle fracture. Acta Mechanica, 94(1-2):1-28, 1992.

[85] G. A. Maugin. Material inhomogeneities in elasticity, volume 3. CRC Press, 1993.

[86] G. A. Maugin. Eshelby stress in elastoplasticity and ductile fracture. International Journal of Plasticity, 10(4):393-408, 1994.

[87] G. A. Maugin. Configurational forces: thermomechanics, physics, mathematics, and numerics. CRC Press, 2010. 
[88] G. Mendel. Versuche über pflanzenhybriden. Verhandlungen des naturforschenden Vereines in Brunn 4: 3, 44, 1866.

[89] W. W. Mullins and R. Sekerka. Stability of a planar interface during solidification of a dilute binary alloy. Journal of applied physics, 35(2):444-451, 1964.

[90] C. D. Murray. The physiological principle of minimum work: I. the vascular system and the cost of blood volume. Proc Natl Acad Sci USA, 12(3):207, 1926.

[91] R. W. Ogden. Non-linear elastic deformations. Courier Corporation, 1997.

[92] A. Papastavrou, P. Steinmann, and E. Kuhl. On the mechanics of continua with boundary energies and growing surfaces. Journal of the Mechanics and Physics of Solids, 61(6):1446-1463, 2013.

[93] S. Pivar. On the origin ofform: evolution by self-organization. North Atlantic Books, 2009.

[94] M. Poujade, E. Grasland-Mongrain, A. Hertzog, J. Jouanneau, P. Chavrier, B. Ladoux, A. Buguin, and P. Silberzan. Collective migration of an epithelial monolayer in response to a model wound. Proceedings of the National Academy of Sciences, 104(41):15988-15993, 2007.

[95] P. Prusinkiewicz, D. R. Fowler, and H. Meinhardt. The algorithmic beauty of sea shells. Springer Science \& Business Media, 2009.

[96] A. Puliafito, L. Hufnagel, P. Neveu, S. Streichan, A. Sigal, D. K. Fygenson, and B. I. Shraiman. Collective and single cell behavior in epithelial contact inhibition. Proceedings of the National Academy of Sciences, 109(3):739744, 2012.

[97] J. Ranft, M. Basan, J. Elgeti, J.-F. Joanny, J. Prost, and F. Jülicher. Fluidization of tissues by cell division and apoptosis. Proceedings of the National Academy of Sciences, 107(49):20863-20868, 2010.

[98] E. K. Rodriguez, A. Hoger, and A. D. McCulloch. Stress-dependent finite growth in soft elastic tissues. J Biomech, 27(4):455-467, 1994.

[99] A. Romano. Thermomechanics of phase transitions in classical field theory, volume 13. World Scientific, 1993.

[100] W. Roux. Beiträge zur entwickelungsmechanik des embryo. Archiv für pathologische Anatomie und Physiologie und für klinische Medicin, 114(2):246-291, 1888.

[101] P. G. Saffman and G. Taylor. The penetration of a fluid into a porous medium or hele-shaw cell containing a more viscous liquid. In Proceedings of the Royal Society of London A: Mathematical, Physical and Engineering Sciences, volume 245, pages 312-329. The Royal Society, 1958.

[102] A. E. Shyer, T. Tallinen, N. L. Nerurkar, Z. Wei, E. S. Gil, D. L. Kaplan, C. J. Tabin, and L. Mahadevan. Villification: how the gut gets its villi. Science, 342(6155):212-218, 2013.

[103] R. Skalak. Growth as a finite displacement field. In Proceedings of the IUTAM Symposium on Finite Elasticity, pages 347-355. Springer, 1982. 
[104] R. Skalak, G. Dasgupta, M. Moss, E. Otten, P. Dullemeijer, and H. Vilmann. Analytical description of growth. Journal of Theoretical Biology, 94(3):555577, 1982.

[105] R. Skalak, S. Zargaryan, R. K. Jain, P. A. Netti, and A. Hoger. Compatibility and the genesis of residual stress by volumetric growth. Journal of Mathematical Biology, 34(8):889-914, 1996.

[106] R. S. Smith, S. Guyomarc'h, T. Mandel, D. Reinhardt, C. Kuhlemeier, and P. Prusinkiewicz. A plausible model of phyllotaxis. Proceedings of the National Academy of Sciences of the United States of America, 103(5):13011306, 2006.

[107] H. Spemann and H. Mangold. Über induktion von embryonalanlagen durch implantation artfremder organisatoren. Development Genes and Evolution, 100(3):599-638, 1924.

[108] M. Stähle, C. Veit, U. Bachfischer, K. Schierling, B. Skripczynski, A. Hall, P. Gierschik, and K. Giehl. Mechanisms in lpa-induced tumor cell migration: critical role of phosphorylated erk. Journal of cell science, 116(18):38353846, 2003.

[109] A. Stathopoulos and D. Iber. Studies of morphogens: keep calm and carry on. Development, 140(20):4119-4124, 2013.

[110] A. Stroh. Steady state problems in anisotropic elasticity. J. math. Phys, 41(2):77-103, 1962.

[111] L. A. Taber. Biomechanics of growth, remodeling, and morphogenesis. Evolution, 490:6, 1995.

[112] D. W. Thompson. On growth and form. Cambridge Univ. Press, 1917.

[113] T. C. Ting and C. Horgan. Anisotropic elasticity: theory and applications. Journal of Applied Mechanics, 63:1056, 1996.

[114] A. M. Turing. The chemical basis of morphogenesis. Philosophical Transactions of the Royal Society of London B: Biological Sciences, 237(641):37-72, 1952.

[115] C. Verdier, J. Etienne, A. Duperray, and L. Preziosi. Review: Rheological properties of biological materials. Comptes Rendus Physique, 10(8):790$811,2009$.

[116] R. L. K. Virchow. Cellular pathology. John Churchill, 1860.

[117] C. H. Waddington. Canalization of development and the inheritance of acquired characters. Nature, 150(3811):563-565, 1942.

[118] J. Wolff. Das gesetz der transformation der knochen. DMW-Deutsche Medizinische Wochenschrift, 19(47):1222-1224, 1892.

[119] L. Wolpert. Positional information and the spatial pattern of cellular differentiation. Journal of theoretical biology, 25(1):1-47, 1969.

[120] L. Wolpert. Positional information and patterning revisited. Journal of Theoretical Biology, 269(1):359-365, 2011.

[121] L. Wolpert, C. Tickle, and A. M. Arias. Principles of development. Oxford university press, 2015. 
[122] M. A. Wozniak and C. S. Chen. Mechanotransduction in development: a growing role for contractility. Nature reviews Molecular cell biology, 10(1):34-43, 2009.

[123] S. R. Yu, M. Burkhardt, M. Nowak, J. Ries, Z. Petrášek, S. Scholpp, P. Schwille, and M. Brand. Fgf8 morphogen gradient forms by a sourcesink mechanism with freely diffusing molecules. Nature, 461(7263):533536, 2009.

[124] E. A. Zamir and L. A. Taber. Material properties and residual stress in the stage 12 chick heart during cardiac looping. Journal of biomechanical engineering, 126(6):823-830, 2004.

[125] A. J. Zhu and M. P. Scott. Incredible journey: how do developmental signals travel through tissue? Genes \& development, 18(24):2985-2997, 2004. 